Portland State University

PDXScholar

\title{
Spontaneous coherent pulsations in standing-wave laser oscillators : stability criteria
}

Pitak Chenkosol

Portland State University

Follow this and additional works at: https://pdxscholar.library.pdx.edu/open_access_etds

Part of the Electrical and Computer Engineering Commons Let us know how access to this document benefits you.

\section{Recommended Citation}

Chenkosol, Pitak, "Spontaneous coherent pulsations in standing-wave laser oscillators : stability criteria" (1992). Dissertations and Theses. Paper 4211.

https://doi.org/10.15760/etd.6095

This Thesis is brought to you for free and open access. It has been accepted for inclusion in Dissertations and Theses by an authorized administrator of PDXScholar. Please contact us if we can make this document more accessible: pdxscholar@pdx.edu. 
AN ABSTRACT OF THE THESIS OF Pitak Chenkosol for the Master of Science in Electrical Engineering presented February 21, 1992.

Title: Spontaneous Coherent Pulsations in Standing-wave Laser Oscillators:

Stability Criteria.

APPROVED BY THE MEMBERS OF THE THESIS COMMITTEE:

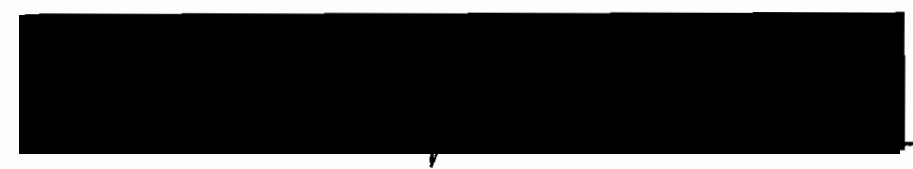

Lee W. Casperson, Chair

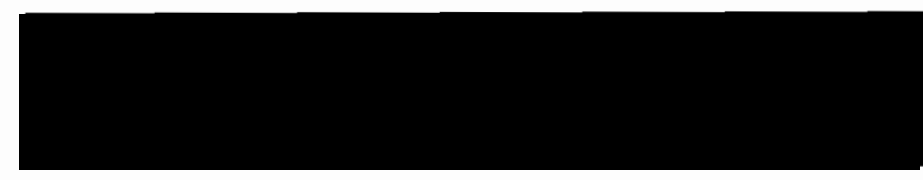

W. Robert Daasch

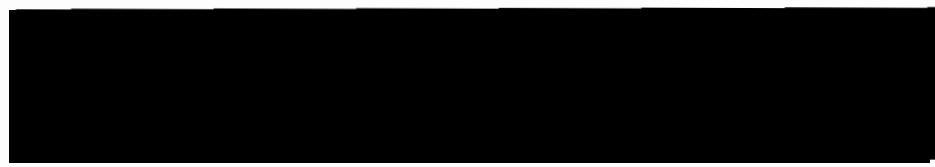

Carl Bachhuber

The stability criteria for single-mode standing-wave laser oscillators in the strongly homogeneously broadened limit are reported for the first time. Two types of stability criteria are presented. The first type, called type 1 , corresponds to the minimum value of threshold parameter for which an infinitesimal perturbation away from steady state grows into an oscillatory solution. Another type of stability criteria, called type 
2, corresponds to the minimum value of threshold parameter for which large amplitude oscillatory solutions do not decay to the steady state solution. Undamped pulsations in single mode strongly homogeneously broadened standing-wave laser oscillators are found to occur at a much higher excitation level than that of ring-laser oscillators with the same type of line broadening. The effect of detuning on stability criteria is also investigated. We discovered that detuning tends to raise the type 1 instability threshold and to decrease the type 2 instability threshold. 


\section{SPONTANEOUS COHERENT PULSATIONS}

IN STANDING-WAVE LASER OSCILLATORS:

STABILITY CRITERIA

by

PITAK CHENKOSOL

A thesis submitted in partial fulfillment of the requirements for the degree of

MASTER OF SCIENCE

in

ELECTRICAL ENGINEERING

Portland State University

1992 
TO THE OFFICE OF GRADUATE STUDIES:

The members of the Committee approve the thesis of Pitak Chenkosol presented February 21, 1992.

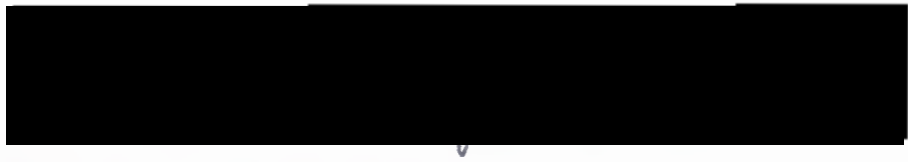

Lee W. Casperson, Chair

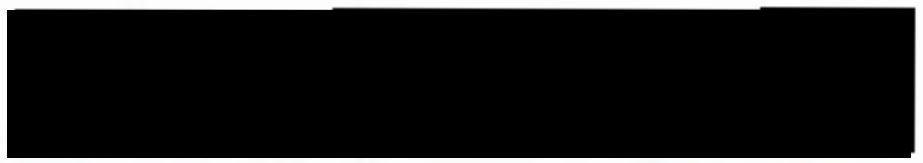

W. Robert Daasch

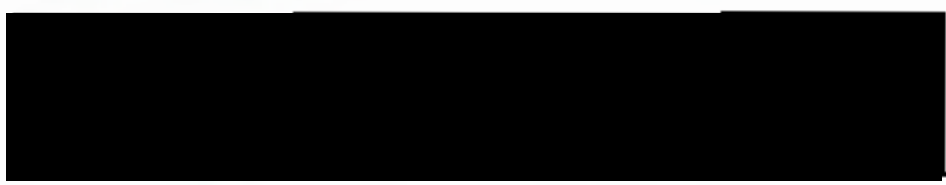

Carl Bachhuber

APPROVED:

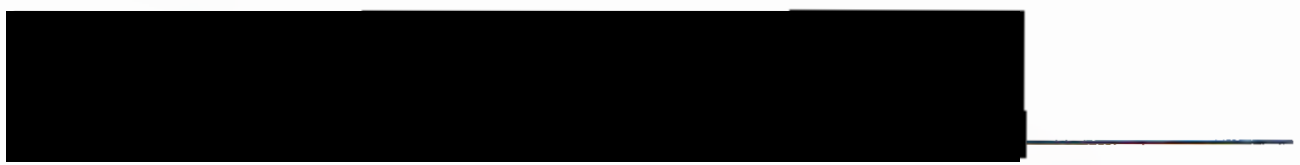

Rolf Schaumann, Chair, Department of Electrical Engineering

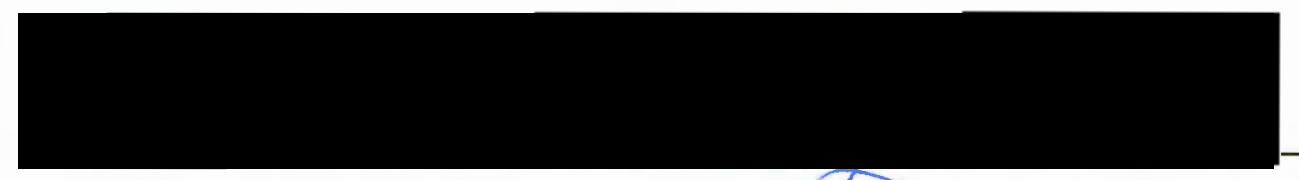

C. William Savery, Vice Provost for Graduate Studies and Research 


\section{ACKNOWLEDGEMENTS}

With this opportunity, I would like to acknowledge the many people who assisted and provided very useful information during the course of this study. First and foremost, I must thank Dr. Lee W. Casperson, my advisor, who not only originated the topic of the study, gave me ideas and encouraged me throughout this period but who also always had answers to my questions and was the major source of references. Many thanks go to Shirley C. Clark and staff of the Department of Electrical Engineering who helped me to overcome the administrative difficulties and to Janaka Jayawardena who provided me with more than a fair share of computing resources and programming tips. Special thanks to the members of the laser research group of the Department of Electrical Engineering at Portland State University for excellent support, especially Anthony A. Tovar for stimulating discussions on the topic. Last but not least is my deep gratitude to my parents who provided a wonderful support while I have been away from home. 


\section{TABLES OF CONTENTS}

PAGE

ACKNOWLEDGEMENTS ............................................................................. iii

LIST OF FIGURES …................................................................................. vi

\section{CHAPTER}

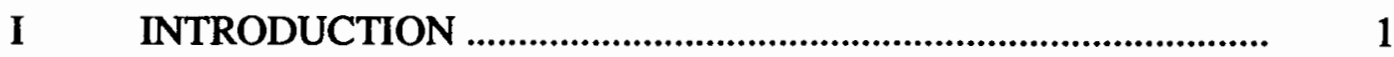

II GENERAL MODEL ................................................................ 5

Theory ............................................................................ 5

Spatial Frequency Expansions ............................................... 14

Normalized Equations............................................................ 18

Monoisotopic Laser Equations ........................................... 21

III SIMPLIFIED MODEL _............................................................ 24

Threshold Parameter ........................................................... 29

Initial Conditions ............................................................ 34

Strongly Homogeneously Broadened Laser Equations ........ $\quad 39$

IV STABILITY ANALYSIS ...................................................... 41

Linear Stability Analysis....................................................... 42

Homogeneous Broadening................................................... 44

Type 1 (Perturbation) Stability............................................... 58

Type 2 Stability ........................................................... 70

Detuning Effect ............................................................ $\quad 70$ 


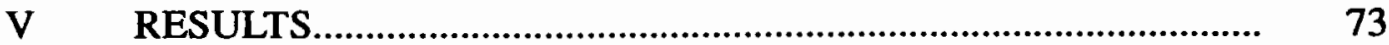

Type 1 (Perturbation) Stability Criteria …............................... 73

Type 2 Stability Criteria ............................................................ 75

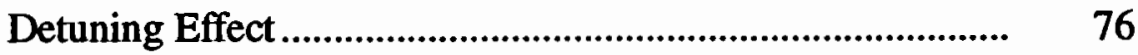

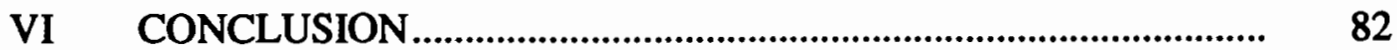

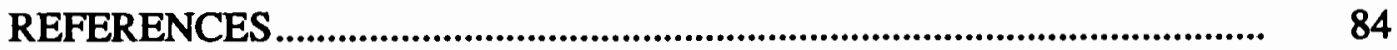




\section{LIST OF FIGURES}

FIGURE

PAGE

1. Flow Chart of Numerical Calculations for Stability

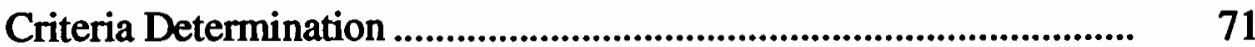

2. Type 1 (Perturbation) Stability Criteria for Standing-wave

Laser Oscillators (Line Center Tuning) ........................................... 74

3. Type 1 (Perturbation) Stability Criteria for Ring-laser

Oscillators (Line Center Tuning) ........................................................ 76

4. Type 2 Stability Criteria for Standing-wave Laser

Oscillators (Line Center Tuning) ........................................................ 77

5. Type 2 Stability Criteria for Ring-laser Oscillators

(Line Center Tuning) .......................................................................... 78

6. Type 1 (Perturbation) Stability Criteria for Standing-wave

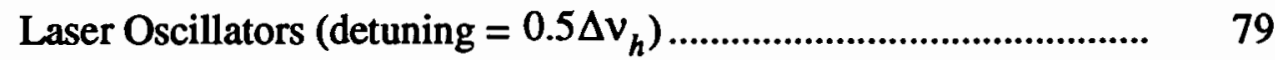

7. Type 2 Stability Criteria for Standing-wave Laser

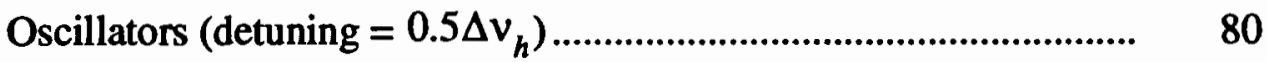

8. Normalized Homogeneously Broadened Gain Profile

$\left(\tau_{2}=\frac{T_{2}}{2}, T_{2}=\right.$ Coherence Time $)$ 


\section{CHAPTER I}

\section{INTRODUCTION}

Instabilities and nonlinear dynamics of lasers are of great theoretical and experimental interests $^{(1),(2)}$. Conditions under which laser instabilities can occur have been given in many places ${ }^{(4)-(7)}$. In [8], Haken showed that, in the resonant tuned case, a single longitudinal mode homogeneously broadened ring laser has the same form of dynamic equations describing the behavior of the system as the most studied lowdimensional convective hydrodynamic system, namely the Lorenz model ${ }^{(9),(10)}$. Theoretical studies of the ring laser model also show results that conform to the results predicted by the Lorenz model ${ }^{(1),(2),(11),(18)-(20)}$. It was discovered that in order to observe pulsation behavior in the homogeneously broadened ring laser system the laser must be operated at an excitation level at least nine times above the laser threshold ${ }^{(4)}$ and must also satisfy the "bad cavity" condition which means that the decay rate of the cavity field is higher than the decay rate of polarization. Because of the "bad cavity" condition, the laser must have high gain in order to overcome the loss due to high cavity field decay rate. Much effort has also been spent in trying to experimentally observe various types of unstable output from homogeneously broadened ring lasers as predicted by the LorenzHaken model, and recently there have been reports of success ${ }^{(12)-(17)}$. In standing-wave lasers, however, the situation is different. Theoretical models for this type of laser ${ }^{(21)-(22)}$ do not show the same similarity to the Lorenz model as those for the ring laser. In [21], Casperson developed a rigorous theoretical model for standing-wave lasers oscillators which provided good agreement between the theoretical and experimental pulsation data of xenon lasers. Our study here is also based on this model. 
The stability criteria for single-mode standing-wave laser oscillators in the strongly homogeneously broadened limit are reported for the first time. The semiclassical laser equations have been solved numerically for different sets of operating parameters. The instability boundaries obtained can be used to qualitatively test whether a continuously pumped laser will produce its output in the form of undamped pulsations. The results of the study are particularly useful for qualitatively testing the behavior of a laser under study even if they cannot provide direct information regarding the pulsation waveforms. This information and the temporal evolution of a laser system can be obtained by direct numerical integration of the dynamic equations governing the behavior of the laser system.

In studies of the dynamic behavior of a laser system, the complexities of the laser equations usually prevent one from solving them analytically even if this can be done in some cases, but the numerical solutions of the laser equations can always be computed. The numerical solutions for homogeneously broadened ring lasers have been known ${ }^{(18),(19)}$ and those of both ring and standing-wave inhomogeneously broadened lasers have also been reported ${ }^{(20)-(22)}$. However, these numerical solutions are costly to carry out, compared to the results if they can be obtained analytically, because they require a considerable amount of computing resources, and after getting the results they can not be applied to other laser systems which correspond to different set of operating parameters. Therefore, it is practically useful to develop some kind of graphical representation that can be applied to broad classes of lasers. An example of such a representation is the stability criteria

In a graphical representation, the stability criteria are curves that show the ranges 
of parameters for which a laser will produce stable continuous wave output and the ranges for which the output will consist of pulsations. Because only a small number of parameter variations can be represented by a two dimensional graph, the laser equations will be simplified so that the graphical representation of the stability criteria is useful.

The stability criteria of both homogeneously and inhomogeneously broadened ring laser oscillators were reported by Casperson ${ }^{(4),(23),(24)}$ and others ${ }^{(6),(7),(25),(26)}$. As a comparison to the former results for ring laser oscillators, it is shown in this study that the minimum value of the excitation level needed for homogeneously broadened standingwave lasers to produce their output in the form of undamped pulsations is considerably higher than that for ring lasers. The results also show the difference between the configuration of instability boundaries in parameter space as compared to the results for the ring cavity laser model.

In this study we emphasize two types of stability criteria that are associated with the nontrivial steady state solutions of the laser equations. The first criterion, which we will call type 1 , corresponds to the minimum value of the threshold parameter for which an infinitesimal perturbation away from the steady state solution grows into an undamped oscillatory solution. The other criterion, which we call type 2 , corresponds to the minimum value of the threshold parameter for which large-amplitude oscillatory solutions do not decay to the steady state solution. As noted ${ }^{(4)}$, the stability of the steady state solution depends on the magnitude of the perturbations to which it is subjected. A large perturbation may cause the onset of pulsations, whereas a small perturbation may not.

The basic dynamic laser equations formulated by Casperson ${ }^{(21)}$ are reviewed in Chapter II. In Chapter III, some simplifications to the dynamic laser equations and 
results from Ref. [22] are reviewed and used. With the introduction of new dimensionless parameters, the simplified laser equations from Ref. [22] are transformed to a simpler set that is appropriate for numerical calculations. The linear stability analysis by which we determine the type 1 stability boundaries for the homogeneous broadened lasers is introduced in Chapter IV. Also included in chapter 4 is the flow chart of the numerical calculations used in this study. The principal results of the study, type 1 and type 2 stability boundaries, are reported in Chapter V. Also presented is graphs showing the effect on the stability boundaries of detuning from line center. We also compare the results of our study to those obtained for ring cavities. One can observe significant differences between the results of the two cavity models. 


\section{CHAPTER II}

\section{GENERAL MODEL}

\section{THEORY}

The starting point in our study is a review of the semiclassical MaxwellSchrödinger laser equations which developed by Casperson $^{(21)}$ for standing-wave laser oscillators. The response of atoms, as a whole, which are subjected to an applied electric field can be conveniently described by the ensemble averaged density matrix equations ${ }^{(21)}$.

$$
\begin{gathered}
\left(\frac{\partial}{\partial t}+v \frac{\partial}{\partial z}\right) \rho_{a b}\left(v, \omega_{\alpha^{\prime}} z, t\right)=-\left(i \omega_{\alpha}+\gamma\right) \rho_{a b}\left(v, \omega_{\alpha}, z, t\right) \\
-\frac{i \mu}{\hbar} E(z, t)\left[\rho_{a a}\left(v, \omega_{b a}, z, t\right)-\rho_{b b}\left(v, \omega_{b \alpha}, z, t\right)\right], \\
\left(\frac{\partial}{\partial t}+v \frac{\partial}{\partial z}\right) \rho_{a a}\left(v, \omega_{\alpha^{\prime}} z, t\right)=\lambda_{a}\left(v, \omega_{\alpha}, z, t\right)-\gamma_{a} \rho_{a a}\left(v, \omega_{\alpha}, z, t\right) \\
+\gamma_{a b} \rho_{a a}\left(v, \omega_{\alpha}, z, t\right)-\left[\frac{i \mu}{\hbar} E(z, t) \rho_{b a}\left(v, \omega_{\alpha^{\prime}}, z, t\right)+c . c\right], \\
-\int_{-\infty}^{\infty} \Gamma_{a}\left(v^{\prime}, v\right) \rho_{a a}\left(v, \omega_{\alpha}, z, t\right) d v^{\prime} \\
+\int_{-\infty}^{\infty} \Gamma_{a}\left(v, v^{\prime}\right) \rho_{a a}\left(v, \omega_{\alpha}, z, t\right) d v^{\prime}, \\
\left(\frac{\partial}{\partial t}+v \frac{\partial}{\partial z}\right) \rho_{b b}\left(v, \omega_{\alpha^{\prime}}, z, t\right)=\lambda_{b}\left(v, \omega_{\alpha}, z, t\right)-\gamma_{b} \rho_{b b}\left(v, \omega_{\alpha}, z, t\right)
\end{gathered}
$$




$$
\begin{aligned}
& +\gamma_{a b} \rho_{a a}\left(v, \omega_{\alpha}, z, t\right)-\left[\frac{i \mu}{\hbar} E(z, t) \rho_{b a}\left(v, \omega_{\alpha}, z, t\right)+c . c .\right] \\
& -\int_{-\infty}^{\infty} \Gamma_{b}\left(v^{\prime}, v\right) \rho_{b b}\left(v, \omega_{\alpha^{\prime}}, z, t\right) d v^{\prime} \\
& +\int_{-\infty}^{\infty} \Gamma_{b}\left(v, v^{\prime}\right) \rho_{b b}\left(v, \omega_{\alpha^{\prime}}, t\right) d v^{\prime} \\
& \rho_{b a}\left(v, \omega_{\alpha}, z, t\right)=\rho_{a b}^{*}\left(v, \omega_{\alpha}, z, t\right),
\end{aligned}
$$

where the subscripts $a$ and $b$ denote the upper and lower laser levels, respectively, $\gamma_{a}$ and $\gamma_{b}$ are the total decay rates for these levels, $\gamma_{a b}$ is the rate of direct decay from level $a$ to level $b, \gamma$ is the decay rate for the off-diagonal elements, $\lambda_{a}$ and $\lambda_{b}$ are the pumping rates, $\omega_{b}$ is the center frequency of the laser transition for members of an atomic or molecular class $\alpha, \mu$ is the electric dipole moment for the laser transition, $\hbar=h /(2 \pi)$ where $h$ is Planck's constant, and the notation c.c. means the complex conjugate of the preceding terms. The integral terms in Eqs.(2.2) and (2.3) represent spectral cross relaxation, and in a low pressure xenon laser this cross relaxation results from velocity-changing collisions. Thus, the function $\Gamma_{a}\left(v^{\prime}, v\right) d v^{\prime}$ indicates the rate at which atoms in level $a$ having velocity between $v$ and $v+d v$ will be bumped by means of collisions into a velocity range between $v^{\prime}$ and $v^{\prime}+d v^{\prime}$. Conversely, the function $\Gamma_{a}\left(v, v^{\prime}\right) d v^{\prime}$ indicates the rate at which atoms in level $a$ will be bumped by means of collisions from all possible velocities back into the range between $v$ and $v+d v$. This spectral cross relaxation effect can also occur in non-Doppler lasers, and the integrals in Eqs.(2.2) and (2.3) could be generalized to included processes that distribute the intrinsic center frequencies, $\omega_{\alpha}$.

The set of density matrix equations above describes the behavior of the atomic or molecular populations and polarizations. An equation for the electric field $E(z, t)$ must be 
added to this set in order to make the whole set self-consistent. The wave equation for the electric field of a linearized polarized wave in a laser medium can be written as

$$
\frac{\partial^{2} E(z, t)}{\partial z^{2}}-\mu_{1} \sigma \frac{\partial E}{\partial t}(z, t)-\mu_{1} \varepsilon_{1} \frac{\partial^{2} E}{\partial t^{2}}(z, t)=\mu_{1} \frac{\partial^{2} P}{\partial t^{2}}(z, t)
$$

The permeability $\mu_{1}$ and permittivity $\varepsilon_{1}$ include all the magnetic and dielectric properties of the laser medium except the polarization $P$, which is due to the lasing atoms or molecules. The polarization driving this field equation can be related to the off-diagonal element of the density matrix by

$$
P(z, t)=\int_{-\infty}^{\infty} \int_{-\infty}^{\infty} \mu \rho_{a b}\left(v, \omega_{\alpha}, z, t\right) d v d \omega_{\alpha}+\text { c.c. }
$$

Equations (2.1)-(2.6) form a complete set from which the time and space dependence of the electric field and of the atomic or molecular parameters can be determined, subject to the boundary conditions at the resonator mirrors. In our study here the solutions of particular interest are those that correspond to standing-wave lasers. By assuming that the losses, e.g. scattering, joule losses, etc., in a standing-wave laser are uniformly distributed, they may be absorbed into the conductivity term. One can also factor out the rapid time variations in Eqs. (2.1)-(2.6) by means of the substitutions

$$
E(z, t)=\frac{1}{2} \sin (k z) E^{\prime}(t) \exp (-i \omega t)+c . c .
$$


and

$$
\rho_{a b}\left(v, \omega_{\alpha}, z, t\right)=\frac{1}{2 \mu} P^{\prime}\left(v, \omega_{\alpha}, z, t\right) \exp (-i \omega t)
$$

where $E^{\prime}(t)$ and $P^{\prime}\left(v, \omega_{\alpha}, z, t\right)$ are slowly varying amplitude functions compared to the exponential terms. The angular frequency $\omega$ would be considered as the actual lasing frequency of the electromagnetic wave in a laser cavity if the amplitude function $E^{\prime}$ is time independent, and the $\sin (k z)$ spatial dependence means that the laser under consideration is assumed to operate in a single longitudinal mode.

By applying the commonly used "rotating wave approximation" these substitutions reduce Eqs.(2.1)-(2.4) to a new set

$$
\begin{gathered}
\left(\frac{\partial}{\partial t}+v \frac{\partial}{\partial z}\right) P^{\prime}\left(v, \omega_{\alpha}, z, t\right)=i\left(\omega-\omega_{\alpha}\right) P^{\prime}\left(v, \omega_{\alpha}, z, t\right)-\gamma P^{\prime}\left(v, \omega_{\alpha}, z, t\right) \\
-\frac{i \mu^{2}}{\hbar} \sin (k z) E^{\prime}(t)\left[\rho_{a a}\left(v, \omega_{\alpha}, z, t\right)-\rho_{b b}\left(v, \omega_{\alpha}, z, t\right)\right] \\
\left(\frac{\partial}{\partial t}+v \frac{\partial}{\partial z}\right) \rho_{a a}\left(v, \omega_{\alpha}, z, t\right)=\lambda_{a}\left(v, \omega_{\alpha}, z, t\right)-\gamma_{a} \rho_{a a}\left(v, \omega_{\alpha}, z, t\right) \\
\frac{i}{4 \hbar} \sin (k z)\left[E\left(t^{\prime}\right) P^{\prime *}\left(v, \omega_{\alpha}, z, t\right)-E^{\prime *}\left(v, \omega_{\alpha}, z, t\right) P^{\prime}\left(v, \omega_{\alpha}, z, t\right)\right] \\
-\int_{-\infty}^{\infty} \Gamma_{a}\left(v^{\prime}, v\right) \rho_{a a}\left(v, \omega_{\alpha}, z, t\right) d v^{\prime} \\
+\int_{-\infty}^{\infty} \Gamma_{a}\left(v, v^{\prime}\right) \rho_{a a}\left(v^{\prime}, \omega_{\alpha}, z, t\right) d v^{\prime} \\
\left(\frac{\partial}{\partial t}+v \frac{\partial}{\partial z}\right) \rho_{b b}\left(v, \omega_{\alpha}, z, t\right)=\lambda_{b}\left(v, \omega_{\alpha}, z, t\right)-\gamma_{b} \rho_{b b}\left(v, \omega_{\alpha^{\prime}}, z, t\right) \\
+\gamma_{a b} \rho_{a d}\left(v, \omega_{\alpha^{\prime}}, z, t\right)
\end{gathered}
$$




$$
\begin{aligned}
& -\frac{i}{4 \hbar} \sin (k z)\left[E^{\prime}(t) P^{\prime *}\left(v, \omega_{\alpha}, z, t\right)-E^{\prime *}(t) P^{\prime}\left(v, \omega_{\alpha}, z, t\right)\right] \\
& -\int_{-\infty}^{\infty} \Gamma_{b}\left(v^{\prime}, v\right) \rho_{b b}\left(v, \omega_{\alpha}, z, t\right) d v^{\prime} \\
& +\int_{-\infty}^{\infty} \Gamma_{b}\left(v, v^{\prime}\right) \rho_{b b}\left(v, \omega_{\alpha}, z, t\right) d v^{\prime}
\end{aligned}
$$

The same substitutions also reduce equation (2.5) to the new field equation

$$
\begin{array}{r}
\sin (k z)\left[i \mu_{1} \varepsilon_{1} \omega \frac{d}{d t} E^{\prime}(t)+\frac{i \mu_{1} \sigma \omega}{2} E^{\prime}(t)+\left(\frac{\omega^{2} \mu_{1} \varepsilon_{1}}{2}-\frac{k^{2}}{2}\right) E^{\prime}(t)\right] \\
=-\frac{\mu_{1} \omega^{2}}{2} \int_{-\infty}^{\infty} \int_{-\infty}^{\infty} P^{\prime}\left(v, \omega_{\alpha}, z, t\right) d v d \omega_{\alpha}
\end{array}
$$

where the field and polarization amplitude functions are assumed to vary slowly with time, as stated earlier, so that their higher order derivatives can be neglected.

By introducing a new frequency parameter $\Omega=k\left(\mu_{1} \varepsilon_{1}\right)^{-1 / 2}$, Eq.(2.12) can be written as

$$
\begin{aligned}
\sin (k z)\left[i \frac{d}{d t} E^{\prime}(t)+\frac{i \sigma}{2 \varepsilon_{1}} E^{\prime}(t)+\frac{\omega^{2}-\Omega^{2}}{2 \omega} E^{\prime}(t)\right] \\
=-\frac{\omega}{2 \varepsilon_{1}} \int_{0}^{\infty} \int_{-\infty}^{\infty} P^{\prime}\left(\nu, \omega_{\alpha}, z, t\right) d v d \omega_{\alpha}
\end{aligned}
$$


The parameter $\Omega$ is the nondispersed cavity frequency, i.e. the steady state lasing frequency if the dispersion or real part of $P^{\prime}$ were equal to zero. Because the assumed lasing frequency $\omega$ is also close to both the nondispersed cavity frequency $\Omega$ and the transition center frequency $\omega_{0}$, the term $\left(\omega^{2}-\Omega^{2}\right) /(2 \omega)$ can be approximated by $(\omega-\Omega)$ and the $\omega$ multiplying the polarization integral can be replaced by $\omega_{0}$. Multiplying Eq.(2.12) by $\sin (k z)$ and integrating over the length $L$ of laser cavity yields

$$
\begin{aligned}
\frac{d}{d t} E^{\prime}(t)=-\frac{\sigma}{2 \varepsilon_{1}} & E^{\prime}(t)+i(\omega-\Omega) E^{\prime}(t) \\
& +\frac{i \omega_{o}}{\mathrm{~L} \varepsilon_{1}} \int_{0}^{\infty} \int_{-\infty}^{\infty} \int_{0}^{\mathrm{L}} \sin (k z) P^{\prime}\left(v, \omega_{\alpha}, z, t\right) d z d v d \omega_{\alpha} .
\end{aligned}
$$

Eqs.(2.9)-(2.11) and (2.14) form a set of first order differential equations governing the laser behavior. These equations are complicated by the fact that the electric field amplitude $E^{\prime}(t)$ and the polarization amplitude $P^{\prime}\left(v, \omega_{\alpha}, z, t\right)$ are complex quantities. For computational purposes later on it will prove helpful to express these quantities in terms of their real and imaginary parts according to

$$
P^{\prime}\left(v, \omega_{\alpha}, z, t\right)=P_{r}\left(v, \omega_{\alpha}, z, t\right)+i P_{i}\left(v, \omega_{\alpha}, z, t\right),
$$

and

$$
E^{\prime}(t)=E_{r}(t)+i E_{i}(t)
$$

With these substitutions the governing equations reduce to a real set 


$$
\begin{aligned}
\left(\frac{\partial}{\partial t}+v \frac{\partial}{\partial z}\right) P_{r}\left(v, \omega_{\alpha}, z, t\right)= & -\left(\omega-\omega_{\alpha}\right) P_{i}\left(v, \omega_{\alpha}, z, t\right)-\gamma P_{r}\left(v, \omega_{\alpha}, z, t\right) \\
& +\frac{\mu^{2}}{\hbar} \sin (k z) E_{i}(t) D\left(v, \omega_{\alpha}, z, t\right), \\
\left(\frac{\partial}{\partial t}+v \frac{\partial}{\partial z}\right) P_{i}\left(v, \omega_{\alpha}, z, t\right)= & \left(\omega-\omega_{\alpha}\right) P_{r}\left(v, \omega_{\alpha}, z, t\right)-\gamma P_{i}\left(v, \omega_{\alpha}, z, t\right) \\
& -\frac{\mu^{2}}{\hbar} \sin (k z) E_{r}(t) D\left(v, \omega_{\alpha}, z, t\right),
\end{aligned}
$$

$$
\begin{aligned}
\left(\frac{\partial}{\partial t}+v \frac{\partial}{\partial z}\right) D & \left(v, \omega_{\alpha}, z, t\right)=\lambda_{a}\left(v, \omega_{\alpha}, z, t\right)-\lambda_{b}\left(v, \omega_{\alpha}, z, t\right) \\
- & \frac{\gamma_{a}+\gamma_{a b}+\gamma_{b}}{2} D\left(v, \omega_{\alpha}, z, t\right)-\frac{\gamma_{a}+\gamma_{a b}+\gamma_{b}}{2} M\left(v, \omega_{\alpha}, z, t\right) \\
& +\frac{\sin (k z)}{\hbar}\left[E_{r}(t) P_{i}\left(v, \omega_{\alpha}, z, t\right)-E_{i}(t) P_{r}\left(v, \omega_{\alpha}, z, t\right)\right] \\
& -\frac{1}{2} \int_{-\infty}^{\infty} \Gamma_{a}\left(v^{\prime}, v\right)\left[M\left(v, \omega_{\alpha}, z, t\right)+D\left(v, \omega_{\alpha}, z, t\right)\right] d v^{\prime} \\
& +\frac{1}{2} \int_{-\infty}^{\infty} \Gamma_{a}\left(v^{\prime}, v\right)\left[M\left(v^{\prime}, \omega_{\alpha}, z, t\right)+D\left(v^{\prime}, \omega_{\alpha}, z, t\right)\right] d v^{\prime} \\
& +\frac{1}{2} \int_{-\infty}^{\infty} \Gamma_{b}\left(v^{\prime}, v\right)\left[M\left(v, \omega_{\alpha}, z, t\right)-D\left(v, \omega_{\alpha}, z, t\right)\right] d v^{\prime} \\
& -\frac{1}{2} \int_{-\infty}^{\infty} \Gamma_{b}\left(v^{\prime}, v\right)\left[M\left(v^{\prime}, \omega_{\alpha}, z, t\right)-D\left(v^{\prime}, \omega_{\alpha}, z, t\right)\right] d v^{\prime}
\end{aligned}
$$

$$
\begin{aligned}
\left(\frac{\partial}{\partial t}+v \frac{\partial}{\partial z}\right) M\left(v, \omega_{\alpha}, z, t\right)=\lambda_{\alpha}\left(v, \omega_{\alpha}, z, t\right)+\lambda_{\alpha}\left(v, \omega_{\alpha}, z, t\right) \\
-\frac{\gamma_{a}-\gamma_{a b}-\gamma_{b}}{2} D\left(v, \omega_{\alpha}, z, t\right)-\frac{\gamma_{a}-\gamma_{a b}+\gamma_{b}}{2} M\left(v, \omega_{\alpha}, z, t\right) \\
-\frac{1}{2} \int_{-\infty}^{\infty} \Gamma_{a}\left(v^{\prime}, v\right)\left[M\left(v, \omega_{\alpha}, z, t\right)+D\left(v, \omega_{\alpha}, z, t\right)\right] d v^{\prime}
\end{aligned}
$$




$$
\begin{aligned}
+ & \frac{1}{2} \int_{-\infty}^{\infty} \Gamma_{a}\left(v^{\prime}, v\right)\left[M\left(v, \omega_{\alpha}, z, t\right)+D\left(v, \omega_{\alpha}, z, t\right)\right] d v^{\prime} \\
& -\frac{1}{2} \int_{-\infty}^{\infty} \Gamma_{b}\left(v, \omega_{\alpha}, z, t\right)\left[M\left(v, \omega_{\alpha}, z, t\right)-D\left(v, \omega_{\alpha}, z, t\right)\right] d v^{\prime} \\
+ & \frac{1}{2} \int_{-\infty}^{\infty} \Gamma_{b}\left(v, \omega_{\alpha}, z, t\right)\left[M\left(v, \omega_{\alpha}, z, t\right)-D\left(v, \omega_{\alpha}, z, t\right)\right] d v^{\prime} \\
\frac{d}{d t} E_{r}(t)=- & \frac{E_{r}(t)}{2 t}-(\omega-\Omega) E_{i}(t) \\
& -\frac{\omega_{o}}{\varepsilon_{1} L} \int_{-\infty}^{\infty} \int_{-\infty}^{\infty} \int_{0}^{l} \sin (k z) P_{i}\left(v, \omega_{\alpha}, z, t\right) d z d v d \omega_{\alpha}, \\
\frac{d}{d t} E_{i}(t)=- & \frac{E_{i}(t)}{2 t_{c}}+(\omega-\Omega) E_{r}(t) \\
& +\frac{\omega_{o}}{\varepsilon_{1} L} \int_{-\infty}^{\infty} \int_{-\infty}^{\infty} \int_{0}^{l} \sin (k z) P_{r}\left(v, \omega_{\alpha}, z, t\right) d z d v d \omega_{\alpha},
\end{aligned}
$$

where we have also introduced the population difference $D\left(v, \omega_{\alpha}, z, t\right)=\rho_{a a}\left(v, \omega_{\alpha}, z, t\right)-\rho_{b b}\left(v, \omega_{\alpha}, z, t\right), \quad$ the population sum $M\left(v, \omega_{\alpha}, z, t\right)=\rho_{a a}\left(v, \omega_{\alpha}, z, t\right)+\rho_{b b}\left(v, \omega_{\alpha}, z, t\right)$, and the cavity lifetime (field intensity decay time) $t_{c}=\varepsilon_{1} / \sigma$. Also, the length of integration extends only over the amplifier length $l$ because outside the amplifier $P^{\prime}\left(v, \omega_{\alpha}, z, t\right)=0$.

At this point it is necessary to make some assumption about the form of the spectral cross-relaxation kernels. We will assume that the spectral cross-relaxation integrals apply only to strong collisions in which the final velocities are distributed randomly across the Doppler profile. The weak collisions are phase interrupting collisions, and we assume that this effect is included in the decay rate $\gamma$. Therefore the spectral cross- 
relaxation kernels can be written as

$$
\begin{array}{ll} 
& \Gamma_{a}\left(v^{\prime}, v\right)=\frac{\Gamma_{a}}{u \pi^{1 / 2}} \exp \left(-v^{2} / u^{2}\right)=\Gamma_{a} W(v), \\
\text { and } \quad \Gamma_{b}\left(v^{\prime}, v\right)=\frac{\Gamma_{b}}{u \pi^{1 / 2}} \exp \left(-v^{2} / u^{2}\right)=\Gamma_{b} W(v),
\end{array}
$$

where $\Gamma_{a}$ and $\Gamma_{b}$ are the total rates at which atoms in level $a$ and $b$ undergo strong velocitychanging collisions, respectively and

$$
W(v)=\frac{1}{u \pi^{1 / 2}} \exp \left(-v^{2} / u^{2}\right)
$$

is the Maxwell-Boltzmann velocity-distribution function with $u$ the most probable speed of the atoms ${ }^{(28)}$. With this substitution for the kernels $\Gamma_{a}\left(v^{\prime}, v\right)$ and $\Gamma_{b}\left(v^{\prime}, v\right)$, Eqs.(2.19) and (2.20) for the population difference and sum become

$$
\begin{aligned}
\left(\frac{\partial}{\partial t}+v \frac{\partial}{\partial z}\right) D\left(v, \omega_{\alpha}, z, t\right)=\lambda_{a}\left(v, \omega_{\alpha}, z, t\right)-\lambda_{b}\left(v, \omega_{\alpha}, z, t\right) \\
-\frac{\gamma_{a}^{\prime}+\gamma_{a b}-\gamma_{b}^{\prime}}{2} D\left(v, \omega_{\alpha}, z, t\right)-\frac{\gamma_{a}^{\prime}+\gamma_{a b}-\gamma_{b}^{\prime}}{2} M\left(v, \omega_{\alpha}, z, t\right) \\
+\frac{\sin (k z)}{\hbar}\left[E_{r}(t) P_{i}\left(v, \omega_{\alpha}, z, t\right)-E_{i}(t) P_{r}\left(v, \omega_{\alpha}, z, t\right)\right]
\end{aligned}
$$




$$
\begin{aligned}
&+ \frac{\Gamma_{a}}{2 u \pi^{1 / 2}} \exp \left(-v^{2} / u^{2}\right) \int_{-\infty}^{\infty}\left[M\left(v^{\prime}, \omega_{\alpha^{\prime}}, z, t\right)+D\left(v^{\prime}, \omega_{\alpha}, z, t\right)\right] d v^{\prime} \\
&- \frac{\Gamma_{b}}{2 u \pi^{1 / 2}} \exp \left(-v^{2} / u^{2}\right) \int_{-\infty}^{\infty}\left[M\left(v^{\prime}, \omega_{\alpha^{\prime}}, z, t\right)-D\left(v^{\prime}, \omega_{\alpha}, z, t\right)\right] d v^{\prime}, \\
&\left(\frac{\partial}{\partial t}+v \frac{\partial}{\partial z}\right) M\left(v, \omega_{\alpha}, z, t\right)=\lambda_{a}\left(v, \omega_{\alpha}, z, t\right)+\lambda_{b}\left(v, \omega_{\alpha}, z, t\right) \\
&-\frac{\gamma_{a}^{\prime}-\gamma_{a b}-\gamma_{b}^{\prime}}{2} D\left(v, \omega_{\alpha}, z, t\right)-\frac{\gamma_{a}^{\prime}-\gamma_{a b}+\gamma_{b}^{\prime}}{2} M\left(v, \omega_{\alpha}, z, t\right) \\
&+\frac{\Gamma_{a}}{2 u \pi^{1 / 2}} \exp \left(-v^{2} / u^{2}\right) \int_{-\infty}^{\infty}\left[M\left(v^{\prime}, \omega_{\alpha}, z, t\right)+D\left(v^{\prime}, \omega_{\alpha}, z, t\right)\right] d v^{\prime} \\
&-\frac{\Gamma_{b}}{2 u \pi^{1 / 2}} \exp \left(-v^{2} / u^{2}\right) \int_{-\infty}^{\infty}\left[M\left(v^{\prime}, \omega_{\alpha}, z, t\right)-D\left(v^{\prime}, \omega_{\alpha}, z, t\right)\right] d v^{\prime},
\end{aligned}
$$

where the new modified decay rates are defined by $\gamma_{a}{ }^{\prime}=\gamma_{a}+\Gamma_{a}$ and $\gamma_{b}{ }^{\prime}=\gamma_{b}+\Gamma_{b}$.

\section{SPATIAL FREQUENCY EXPANSIONS}

One difficulty with the model described by Eqs. (2.17), (2.18), (2.21), (2.22), (2.26), and (2.27) is that it includes partial derivatives with respect to both space and time variables. Because of the high spatial frequency of the fields in the cavity of most lasers it is efficient to expand the polarization and the populations into the series of spatial harmonics of the electric field. Thus we introduce the expansion

$$
P_{r}\left(v, \omega_{\alpha}, z, t\right)=\sum_{j=-\infty}^{\infty} P_{r, 2 j+1}\left(v, \omega_{\alpha}, t\right) \exp [(2 j+1) i k z],
$$




$$
\begin{aligned}
& P_{i}\left(v, \omega_{\alpha}, z, t\right)=\sum_{j=-\infty}^{\infty} P_{i, 2 j+1}\left(v, \omega_{\alpha}, t\right) \exp [(2 j+1) i k z], \\
& D\left(v, \omega_{\alpha}, z, t\right)=\sum_{j=-\infty}^{\infty} D_{2 j}\left(v, \omega_{\alpha}, t\right) \exp [(2 j) i k z], \\
& M\left(v, \omega_{\alpha}, z, t\right)=\sum_{j=-\infty}^{\infty} M_{2 j}\left(v, \omega_{\alpha}, t\right) \exp [(2 j) i k z],
\end{aligned}
$$

where only odd harmonics of the polarizations and even harmonics of the populations are included. In this study we make the usual assumption that the pump rates are spatially uniform, and thus no additional harmonics are required. Additionally, the above spatial expansions also imply a high value of cavity mirror reflectivities (or small value of the mirror transmission coefficients).

For the left-hand side of Eqs.(2.28)-(2.31) to be real, additional constraints on the expansion coefficients are required. These constraints can be written as

$$
\begin{aligned}
& P_{r, j}\left(v, \omega_{\alpha}, t\right)=P_{r,-j}^{*}\left(v, \omega_{\alpha}, t\right) \\
& P_{i, j}\left(v, \omega_{\alpha}, t\right)=P_{i,-j}^{*}\left(v, \omega_{\alpha}, t\right) \\
& D_{j}\left(v, \omega_{\alpha}, t\right)=D_{-j}^{*}\left(v, \omega_{\alpha}, t\right) \\
& M_{j}\left(v, \omega_{\alpha}, t\right)=M_{-j}^{*}\left(v, \omega_{\alpha}, t\right)
\end{aligned}
$$

With the substitution of Eqs.(2.32)-(2.35) into Eqs.(2.17), (2.18), (2.21), (2.22), 
16

(2.26), and (2.27) one can obtain

$$
\begin{aligned}
\frac{\partial}{\partial t} P_{r, 2 j+1}\left(v, \omega_{\alpha}, t\right)= & -\left(\omega-\omega_{\alpha}\right) P_{i, 2 j+1}\left(v, \omega_{\alpha}, t\right) \\
& -[(2 j+1) i k v+\gamma] P_{r, 2 j+1}\left(v, \omega_{\alpha}, t\right) \\
& -\frac{i \mu^{2}}{2 \hbar} E_{i}(t)\left[D_{2 j}\left(v, \omega_{\alpha}, t\right)-D_{2 j+2}\left(v, \omega_{\alpha}, t\right)\right], \\
\frac{\partial}{\partial t} P_{i, 2 j+1}\left(v, \omega_{\alpha}, t\right)= & \left(\omega-\omega_{\alpha}\right) P_{r, 2 j+1}\left(v, \omega_{\alpha}, t\right) \\
& -[(2 j+1) i k v+\gamma] P_{i, 2 j+1}\left(v, \omega_{\alpha}, t\right) \\
& +\frac{i \mu^{2}}{2 \hbar} E_{r}(t)\left[D_{2 j}\left(v, \omega_{\alpha}, t\right)-D_{2 j+2}\left(v, \omega_{\alpha}, t\right)\right],
\end{aligned}
$$

$$
\begin{aligned}
& \frac{\partial}{\partial t} D_{2 j}\left(v, \omega_{\alpha}, t\right)=\left[\lambda_{a}\left(v, \omega_{\alpha}, t\right)-\lambda_{b}\left(v, \omega_{\alpha}, t\right)\right] \delta_{j 0} \\
&-\left[(2 j+1) i k v+h_{1}\right] D_{2 j}\left(v, \omega_{\alpha}, t\right)-h_{2} M_{2 j}\left(v, \omega_{\alpha}, t\right) \\
&-\frac{i}{2 h}\left\{\left[E_{r}(t) P_{i, 2 j-1}\left(v, \omega_{\alpha}, t\right)-E_{i}(t) P_{r, 2 j-1}\left(v, \omega_{\alpha}, t\right)\right]\right. \\
&\left.-\left[E_{r}(t) P_{i, 2 j+1}\left(v, \omega_{\alpha}, t\right)-E_{i}(t) P_{r, 2 j+1}\left(v, \omega_{\alpha}, t\right)\right]\right\} \\
&+\frac{\Gamma_{a}}{2 u \pi^{1 / 2}} \exp \left(-v^{2} / u^{2}\right) \int_{-\infty}^{\infty}\left[M_{2 j}\left(v^{\prime}, \omega_{\alpha}, t\right)+D_{2 j}\left(v^{\prime}, \omega_{\alpha}, t\right)\right] d v^{\prime} \\
&-\frac{\Gamma_{b}}{2 u \pi^{1 / 2}} \exp \left(-v^{2} / u^{2}\right) \int_{-\infty}^{\infty}\left[M_{2 j}\left(v^{\prime}, \omega_{\alpha}, t\right)-D_{2 j}\left(v^{\prime}, \omega_{\alpha}, t\right)\right] d v^{\prime},(2)
\end{aligned}
$$

$$
\frac{\partial}{\partial t} M_{2 j}\left(v, \omega_{\alpha}, t\right)=\left[\lambda_{a}\left(v, \omega_{\alpha}, t\right)+\lambda_{b}\left(v, \omega_{\alpha}, t\right)\right] \delta_{j 0}
$$




$$
\begin{aligned}
-\left[(2 j) i k v+h_{3}\right] M_{2 j}\left(v, \omega_{\alpha}, t\right)-h_{4} D_{2 j}\left(v, \omega_{\alpha}, t\right) \\
+\frac{\Gamma_{a}}{2 u \pi^{1 / 2}} \exp \left(-v^{2} / u^{2}\right) \int_{-\infty}^{\infty}\left[M_{2 j}\left(v^{\prime}, \omega_{\alpha}, t\right)+D_{2 j}\left(v^{\prime}, \omega_{\alpha}, t\right)\right] d v^{\prime} \\
+\frac{\Gamma_{b}}{2 u \pi^{1 / 2}} \exp \left(-v^{2} / u^{2}\right) \int_{-\infty}^{\infty}\left[M_{2 j}\left(v^{\prime}, \omega_{\alpha}, t\right)-D_{2 j}\left(v^{\prime}, \omega_{\alpha}, t\right)\right] d v^{\prime}, \\
\frac{d}{d t} E_{r}(t)=-\frac{E_{r}(t)}{2 t_{c}}-(\omega-\Omega) E_{i}(t) \\
\quad-\frac{\omega_{0} l}{\varepsilon_{1} L} \int_{0}^{\infty} \int_{-\infty}^{\infty} \frac{P_{i,-1}\left(v, \omega_{\alpha}, t\right)-P_{i, 1}\left(v, \omega_{\alpha}, t\right)}{2 i} d v d \omega_{\alpha} \\
\frac{d}{d t} E_{i}(t)=-\frac{E_{i}(t)}{2 t_{c}}+(\omega-\Omega) E_{r}(t) \\
+\frac{\omega_{0} l}{\varepsilon_{1} L} \int_{0}^{\infty} \int_{-\infty}^{\infty} \frac{P_{r,-1}\left(v, \omega_{\alpha}, t\right)-P_{r, 1}\left(v, \omega_{\alpha}, t\right)}{2 i} d v d \omega_{\alpha}
\end{aligned}
$$

where the decay rates have been replaced by a new hybrid set

$$
\begin{aligned}
& h_{1}=\frac{\left(\gamma_{a}^{\prime}+\gamma_{a b}+\gamma_{b}^{\prime}\right)}{2}, \\
& h_{2}=\frac{\left(\gamma_{a}^{\prime}+\gamma_{a b}-\gamma_{b}^{\prime}\right)}{2}, \\
& h_{3}=\frac{\left(\gamma_{a}^{\prime}-\gamma_{a b}+\gamma_{b}^{\prime}\right)}{2},
\end{aligned}
$$




$$
h_{4}=\frac{\left(\gamma_{a}^{\prime}-\gamma_{a b}-\dot{\gamma}_{b}\right)}{2}
$$

The $z$ integrations in the field equations have selected out the -1 and +1 spatial harmonics of the polarizations, because the higher-order components averaged out to zero over long distances compared to the wavelength of the laser field.

\section{NORMALIZED EQUATIONS}

It is helpful to write the equations in a normalized form, one possibility is

$$
\begin{aligned}
\frac{\partial}{\partial t} P_{r, 2 j+1}(V, U, t)= & -\gamma\left\{[1+(2 j+1) i V] P_{r, 2 j+1}(V, U, t)\right. \\
& +(y-U) P_{i, 2 j+1}(V, U, t) \\
& \left.+i A_{i}(t)\left[D_{2 j}(V, U, t)-D_{2 j+2}(V, U, t)\right]\right\},
\end{aligned}
$$

$$
\frac{\partial}{\partial t} P_{i, 2 j+1}(V, U, t)=-\gamma\left\{[1+(2 j+1) i V] P_{i, 2 j+1}(V, U, t)\right.
$$

$$
\begin{aligned}
& -(y-U) P_{r, 2 j+1}(V, U, t) \\
& \left.-i A_{r}(t)\left[D_{2 j}(V, U, t)-D_{2 j+2}(V, U, t)\right]\right\},
\end{aligned}
$$

$$
\begin{aligned}
& \frac{\partial}{\partial t} D_{2 j}(V, U, t)=\left[\lambda_{a}(V, U, t)-\lambda_{b}(V, U, t)\right] \delta_{j 0} \\
&-\left[h_{1}+(2 j) i \gamma V\right] D_{2 j}(V, U, t)-h_{2} M_{2 j}(V, U, t) \\
&-i \gamma_{1}\left(\left[A_{r}(t) P_{i, 2 j-1}(V, U, t)-A_{i}(t) P_{r, 2 j-1}(V, U, t)\right]\right. \\
&\left.-\left[A_{r}(t) P_{i, 2 j+1}(V, U, t)-A_{i}(t) P_{r, 2 j+1}(V, U, t)\right]\right\}
\end{aligned}
$$




$$
\begin{aligned}
& +\frac{\varepsilon \Gamma_{a}}{2 \pi^{1 / 2}} \exp \left(-\varepsilon^{2} V^{2}\right) \int_{-\infty}^{\infty}\left[M_{2 j}\left(V^{\prime}, U, t\right)+D_{2 j}\left(V^{\prime}, U, t\right)\right] d V^{\prime} \\
& -\frac{\varepsilon \Gamma_{b}}{2 \pi^{1 / 2}} \exp \left(-\varepsilon^{2} V^{2}\right) \int_{-\infty}^{\infty}\left[M_{2 j}\left(V^{\prime}, U, t\right)-D_{2 j}\left(V^{\prime}, U, t\right)\right] d V^{\prime},(2.48)
\end{aligned}
$$$$
\frac{\partial}{\partial t} M_{2 j}(V, U, t)=\left[\lambda_{a}(V, U, t)+\lambda_{b}(V, U, t)\right] \delta_{j 0}
$$$$
-\left[h_{3}+(2 j) i \gamma V\right] M_{2 j}(V, U, t)-h_{4} D_{2 j}(V, U, t)
$$$$
+\frac{\varepsilon \Gamma_{a}}{2 \pi^{1 / 2}} \exp \left(-\varepsilon^{2} V^{2}\right) \int_{-\infty}^{\infty}\left[M_{2 j}\left(V^{\prime}, U, t\right)+D_{2 j}\left(V^{\prime}, U, t\right)\right] d V^{\prime}
$$$$
+\frac{\varepsilon \Gamma_{b}}{2 \pi^{1 / 2}} \exp \left(-\varepsilon^{2} V^{2}\right) \int_{-\infty}^{\infty}\left[M_{2 j}\left(V^{\prime}, U, t\right)-D_{2 j}\left(V^{\prime}, U, t\right)\right] d V^{\prime},(2.49)
$$$$
\frac{d}{d t} A_{r}(t)=-\frac{1}{2 t_{c}}\left[\left(A_{r}(t)+\delta\left(y-y_{0}\right) A_{i}(t)\right)\right.
$$$$
\left.-\int_{-\infty}^{\infty} \int_{-\infty}^{\infty} P_{i, 1 i}(V, U, t) d V d U\right]
$$$$
\frac{d}{d t} A_{i}(t)=-\frac{1}{2 t_{c}}\left[\left(A_{i}(t)-\delta\left(y-y_{0}\right) A_{r}(t)\right)\right.
$$$$
\left.+\int_{-\infty}^{\infty} \int_{-\infty}^{\infty} P_{i, 1 i}(V, U, t) d V d U\right]
$$

where the new variables

$$
V=\frac{v}{\varepsilon u}=\frac{k v}{\gamma}
$$




$$
\begin{aligned}
& U=\frac{\left(\omega_{\alpha}-\omega_{0}\right)}{\gamma}, \\
& y=\frac{\left(\omega-\omega_{0}\right)}{\gamma}, \\
& y_{0}=\frac{\left(\Omega-\omega_{0}\right)}{\gamma}, \\
& \gamma_{1}=\frac{2 \gamma_{a}^{\prime} \gamma_{b}^{\prime}}{\left(\gamma_{a}^{\prime}-\gamma_{a b}+\gamma_{b}^{\prime}\right)}, \\
& \delta=2 \gamma t_{c},
\end{aligned}
$$

and the normalized field components

$$
\begin{aligned}
A_{r} & =\frac{\mu}{2 \hbar}\left[\frac{h_{3}}{\left(h_{1} h_{3}-h_{2} h_{4}\right) \gamma}\right]^{1 / 2} E_{r} \\
& =\frac{\mu}{2 \hbar}\left[\frac{\gamma_{a}{ }^{\prime}-\gamma_{a b}+\gamma_{b}{ }^{\prime}}{2 \gamma \gamma_{a}{ }^{\prime} \gamma_{b}{ }^{\prime}}\right]^{1 / 2} E_{r}, \\
A_{i} & =\frac{\mu}{2 \hbar}\left[\frac{h_{3}}{\left(h_{1} h_{3}-h_{2} h_{4}\right) \gamma}\right]^{1 / 2} E_{i} \\
& =\frac{\mu}{2 \hbar}\left[\frac{\gamma_{a}{ }^{\prime}-\gamma_{a b}+\gamma_{b}{ }^{\prime}}{2 \gamma \gamma_{a}{ }^{\prime} \gamma_{b}{ }^{\prime}}\right]^{1 / 2} E_{i},
\end{aligned}
$$

have been introduced. The parameter $\varepsilon=\left(\left(\Delta v_{h}\right) /\left(\Delta v_{d}\right)\right)(\ln 2)^{1 / 2}$ is the natural 
damping ratio ${ }^{(30)}$ which measures the relative magnitude of the homogeneous and the inhomogeneous linewidths and the parameter $\delta=2 \gamma t_{c}$ measures the ratio of the polarization and the electric field decay rates. The Guassian velocity distribution in the pump functions has also been factored out according to

$$
\begin{aligned}
& \lambda_{a}\left(v, \omega_{a}\right)=\frac{L_{a}\left(\omega_{\alpha}\right)}{u \pi^{1 / 2}} \exp \left(-v^{2} / u^{2}\right), \\
& \lambda_{b}\left(v, \omega_{a}\right)=\frac{L_{b}\left(\omega_{\alpha}\right)}{u \pi^{1 / 2}} \exp \left(-v^{2} / u^{2}\right),
\end{aligned}
$$

where $L_{a}$ and $L_{b}$ are total pump rates to the upper and lower laser levels respectively. The symbol $\delta_{j 0}$ is the so-called "Kronecker Delta" which has the property

$$
\delta_{j 0}= \begin{cases}1 & \text { if } j=0 \\ 0 & \text { if } j \neq 0\end{cases}
$$

\section{MONOISOTOPIC LASER EQUATIONS}

Equations (2.46)-(2.51) form a general set which allows for the possibility of a distribution of the natural transition center frequency ( $U$ in the normalized units). The value of this normalized transition center frequency, $U$ can also be negative, and the lower limit of the integration over $U$ has been extended to minus infinity. In our study here we will concentrate particularly on the stability criteria of monoisotopic lasers such as xenon laser. The result of this is that the polarization and the population variables may be regarded as delta functions of the parameter $U$ and they can be replaced with a new set of 
variables $^{(21)}$

$$
\begin{aligned}
& P_{r, 2 j+1}(V, t)=\int_{-\infty}^{\infty} P_{r, 2 j+1}(V, t) \delta(U) d U=\int_{-\infty}^{\infty} P_{r, 2 j+1}(V, U, t) d U, \\
& P_{i, 2 j+1}(V, t)=\int_{-\infty}^{\infty} P_{i, 2 j+1}(V, t) \delta(U) d U=\int_{-\infty}^{\infty} P_{i, 2 j+1}(V, U, t) d U, \\
& D_{2 j}(V, t)=\int_{-\infty}^{\infty} D_{2 j}(V, t) \delta(U) d U=\int_{-\infty}^{\infty} D_{2 j}(V, U, t) d U, \\
& M_{2 j}(V, t)=\int_{-\infty}^{\infty} M_{2 j}(V, t) \delta(U) d U=\int_{-\infty}^{\infty} M_{2 j}(V, U, t) d U \\
& \lambda_{a}(V, t)=\int_{-\infty}^{\infty} \lambda_{a}(V, t) \delta(U) d U=\int_{-\infty}^{\infty} \lambda_{a}(V, U, t) d U \\
& \lambda_{b}(V, t)=\int_{-\infty}^{\infty} \lambda_{b}(V, t) \delta(U) d U=\int_{-\infty}^{\infty} \lambda_{b}(V, U, t) d U
\end{aligned}
$$

where $\delta(U)$ is the Dirac delta function in the $U$ variable. With these variable substitutions and with $U$ set equal to zero, Eqs.(2.46)-(2.51) reduce to

$$
\begin{aligned}
\frac{\partial}{\partial t} P_{r, 2 j+1}(V, t)= & -\gamma\left\{[1+(2 j+1) i V] P_{r, 2 j+1}(V, t)+y P_{i, 2 j+1}(V, t)\right. \\
& \left.+i A_{i}(t)\left[D_{2 j}(V, t)-D_{2 j+2}(V, t)\right]\right\} \\
\frac{\partial}{\partial t} P_{i, 2 j+1}(V, t)= & -\gamma\left\{[1+(2 j+1) i V] P_{i, 2 j+1}(V, t)-y P_{i, 2 j+1}(V, t)\right. \\
& \left.-i A_{r}(t)\left[D_{2 j}(V, t)-D_{2 j+2}(V, t)\right]\right\} \\
\frac{\partial}{\partial t} D_{2 j}(V, t)=\left[\lambda_{a}(V, t)-\lambda_{b}(V, t)\right] \delta_{j 0}-\left[h_{1}+(2 j) i \gamma V\right] D_{2 j}(V, t) & \\
- & h_{2} M_{2 j}(V, t)-i \gamma_{1}\left[A_{r}(t) P_{i, 2 j-1}(V, t)-A_{i}(t) P_{r, 2 j-1}(V, t)\right]
\end{aligned}
$$




$$
\begin{gathered}
\left.-\left[A_{r}(t) P_{i, 2 j+1}(V, t)-A_{i}(t) P_{r, 2 j+1}(V, t)\right]\right\} \\
+\frac{\varepsilon \Gamma_{a}}{2 \pi^{1 / 2}} \exp \left(-\varepsilon^{2} V^{2}\right) \int_{-\infty}^{\infty}\left[M_{2 j}\left(V^{\prime}, t\right)+D_{2 j}\left(V^{\prime}, t\right)\right] d V^{\prime} \\
-\frac{\varepsilon \Gamma_{b}}{2 \pi^{1 / 2}} \exp \left(-\varepsilon^{2} V^{2}\right) \int_{-\infty}^{\infty}\left[M_{2 j}\left(V^{\prime}, t\right)-D_{2 j}\left(V^{\prime}, t\right)\right] d V^{\prime}, \\
\frac{\partial}{\partial t} M_{2 j}(V, t)=\left[\lambda_{a}(V, t)+\lambda_{b}(V, t)\right] \delta_{j 0}-\left[h_{3}+(2 j) i \gamma V\right] M_{2 j}(V, t) \\
-h_{4} D_{2 j}(V, t) \\
+\frac{\varepsilon \Gamma_{a}}{2 \pi^{1 / 2}} \exp \left(-\varepsilon^{2} V^{2}\right) \int_{-\infty}^{\infty}\left[M_{2 j}\left(V^{\prime}, t\right)+D_{2 j}\left(V^{\prime}, t\right)\right] d V^{\prime} \\
+\frac{\varepsilon \Gamma_{b}}{2 \pi^{1 / 2}} \exp \left(-\varepsilon^{2} V^{2}\right) \int_{-\infty}^{\infty}\left[M_{2 j}\left(V^{\prime}, t\right)-D_{2 j}\left(V^{\prime}, t\right)\right] d V^{\prime}, \\
\frac{1}{d t} A_{r}(t)=-\frac{1}{2 t_{c}}\left[A_{r}(t)+\delta\left(y-y_{0}\right) A_{i}(t)-\int_{-\infty}^{\infty} P_{i, 1 i}(V, t) d V\right], \\
A_{i}(t)=-\frac{1}{2 t}\left[A_{i}(t)-\delta\left(y-y_{0}\right) A_{r}(t)+\int_{-\infty}^{\infty} P_{r, 1 i}(V, t) d V\right] . \\
\end{gathered}
$$

This reduced form of the laser equations is the basis for our stability analysis in this study. 


\section{CHAPTER III}

\section{SIMPLIFIED MODEL}

The general laser model obtained earlier still contains more variables than necessary to determine the qualitative pulsation behavior of the system. At first glance one may observe that the difficulty in finding the solutions for this set of equations results from the large number of equations involved. This is inevitable because of the inherent complication of the laser system even in its simplest configuration. For example, one must consider a whole new set of equations for each velocity class of moving atoms or molecules of the laser medium in the inhomogeneously broadened gas laser. As previously studied ${ }^{(21)}$, at least a hundred separate velocity classes must be considered in order to accurately represent the pulsation phenomena in a xenon laser. Another complication arises from the large number of spatial harmonics which are necessary for the explanation of some effects that are specific to standing-wave lasers, such as spatial hole burning. We find that at high values of saturation one may need to retain the number of the spatial harmonic up to 18.

Before we proceed any further it is helpful to make some simplification to the general laser model above so that it will contain the minimum number of parameters that determine the pulsation behavior of the laser system but still give a qualitative agreement between theoretical and experimental results. In this chapter we will review and use the results of simplification of Ref. [22]. The simplification will help speed up the computation time, but when one needs quantitative agreement between the theoretical and the experimental results the general laser model is inevitably required. 
One major complication in the model is the inclusion of spectral cross-relaxation, which is an important effect in achieving quantitative agreement with data obtained using xenon lasers. The noticeable effects of the spectral cross-relaxation are to speed up the pulsations and to stabilize them ${ }^{(21)}$. In addition, numerical averaging of laser intensity also shows that ${ }^{(21)}$ the average intensity is higher when the spectral cross-relaxation is included. This is an expected result because with the inclusion of the spectral cross-relaxation, the saturating fields in the laser cavity can interact with a larger number of atoms or molecules of the amplifying medium. For the qualitative investigation purposes here we choose to switch off the spectral-cross relaxation effect by setting $\Gamma_{a}=\Gamma_{b}=0$, the result of this is simpler equations for the parameters $D_{2 j}(V, t)$ and $M_{2 j}(V, t)$, Eqs. (2.71) and (2.72), which can be rewritten as ${ }^{(22)}$

$$
\begin{aligned}
\frac{\partial}{\partial t} D_{2 j}(V, t)= & {\left[\lambda_{a}(V, t)-\lambda_{b}(V, t)\right] \delta_{j 0}-\left[h_{1}+(2 j) i \gamma V\right] D_{2 j}(V, t) } \\
- & h_{2} M_{2 j}(V, t)-i \gamma_{1}\left[A_{r}(t) P_{i, 2 j-1}(V, t)-A_{i}(t) P_{r, 2 j-1}(V, t)\right] \\
& \left.-A_{r}(t) P_{i, 2 j+1}(V, t)+A_{i}(t) P_{r, 2 j+1}(V, t)\right]
\end{aligned}
$$

and

$$
\begin{aligned}
\frac{\partial}{\partial t} M_{2 j}(V, t)= & {\left[\lambda_{a}(V, t)+\lambda_{b}(V, t)\right] \delta_{j 0}-\left[h_{3}+(2 j) i \gamma V\right] M_{2 j}(V, t) } \\
& -h_{4} D_{2 j}(V, t)
\end{aligned}
$$

Another complication of the general model concerns the arbitrary energy-level model that has been chosen. The general laser model allows for arbitrary decay rates from each laser level and also an arbitrary decay rate between the upper and lower laser levels. As previously shown ${ }^{(22)}$, if $\gamma_{b}=\gamma_{a}+\gamma_{a b}$, then the population sum parameter, $M$ and its dynamical equation, Eqs.(3.2), can be ignored from the general set because Eq. (3.1) 
becomes independent of the parameter $M_{2 j}$. This will also help reduce the number of equations involved in the computation. It was shown that these simplified energy decay rates have little qualitative effect except for the laser operation close to threshold ${ }^{(22)}$. By setting $\gamma_{b}=\gamma_{a}+\gamma_{a b}=\gamma_{d}$, and $\Gamma_{a}=\Gamma_{b}=0$, the hybrid decay rate $h_{1}$ simplifies to

$$
\begin{aligned}
h_{1} & =\frac{\left(\gamma_{a}^{\prime}+\gamma_{a b}+\gamma_{b}{ }^{\prime}\right)}{2}, \\
& =\frac{\left(\gamma_{a}+\Gamma_{a}\right)+\gamma_{a b}+\left(\gamma_{b}+\Gamma_{b}\right)}{2}, \\
& =\gamma_{d} .
\end{aligned}
$$

This $\gamma_{d}$ will be shown shortly to be the "population decay rate". With these simplifications the general laser model can be rewritten as ${ }^{(22)}$ :

$$
\begin{aligned}
& \frac{\partial}{\partial t} P_{r, 2 j+1}(V, t)=-\gamma\left[[1+(2 j+1) i V] P_{r, 2 j+1}(V, t)+y P_{i, 2 j+1}(V, t)\right. \\
&\left.+i A_{i}(t)\left[D_{2 j}(V, t)-D_{2 j+2}(V, t)\right]\right\}, \\
& \frac{\partial}{\partial t} P_{i, 2 j+1}(V, t)=-\gamma\left\{[1+(2 j+1) i V] P_{i, 2 j+1}(V, t)-y P_{r, 2 j+1}(V, t)\right. \\
&\left.+i A_{r}(t)\left[D_{2 j}(V, t)-D_{2 j+2}(V, t)\right]\right\}, \\
& \frac{\partial}{\partial t} D_{2 j}(V, t)=\left[\lambda_{a}(V, t)-\lambda_{b}(V, t)\right] \delta_{j 0}-\left[\gamma_{d}+(2 j) i \gamma V\right] D_{2 j}(V, t)-i \gamma_{d}\left[\left(A_{r}(t) P_{i, 2 j-1}(V, t)-A_{i}(t) P_{r, 2 j-1}(V, t)\right)\right. \\
&-\left.-A_{r}(t) P_{i, 2 j+1}(V, t)+A_{i}(t) P_{r, 2 j+1}(V, t)\right], \\
& \frac{d}{d t} A_{r}(t)=-\gamma_{c}\left[A_{r}(t)+\delta\left(y-y_{0}\right) A_{i}(t)-\int_{-\infty}^{\infty} P_{i, 1 i}(V, t) d V\right],
\end{aligned}
$$




$$
\frac{d}{d t} A_{i}(t)=-\gamma_{c}\left[A_{i}(t)-\delta\left(y-y_{0}\right) A_{r}(t)+\int_{-\infty}^{\infty} P_{r, 1 i}(V, t) d V\right]
$$

From Eq. (3.6) for $j=0$ and assuming no field, one can easily see that $\gamma_{d}$ is the decay rate of the population parameter, $D_{2 j}$. For numerical calculation purposes and for easier graphical display of the stability contours, it proves convenient to introduce the dimensionless parameters ${ }^{(4)} \delta=\gamma / \gamma_{c}, \rho=\gamma_{d} / \gamma$, and $t^{\prime}=\gamma_{c} t$. With these definitions, Eqs.(3.4)-(3.8) can be written as

$$
\begin{aligned}
& \frac{\partial}{\partial t^{\prime}} P_{r, 2 j+1}\left(V, t^{\prime}\right)=-\delta\left\{[1+(2 j+1) i V] P_{r, 2 j+1}\left(V, t^{\prime}\right)+y P_{i, 2 j+1}\left(V, t^{\prime}\right)\right. \\
& \left.+i A_{i}\left(t^{\prime}\right)\left[D_{2 j}\left(V, t^{\prime}\right)-D_{2 j+2}\left(V, t^{\prime}\right)\right]\right\} \\
& \frac{\partial}{\partial t^{\prime}} P_{i, 2 j+1}\left(V, t^{\prime}\right)=-\delta\left\{[1+(2 j+1) i V] P_{i, 2 j+1}\left(V, t^{\prime}\right)-y P_{r, 2 j+1}\left(V, t^{\prime}\right)\right. \\
& \left.+i A_{r}\left(t^{\prime}\right)\left[D_{2 j}\left(V, t^{\prime}\right)-D_{2 j+2}\left(V, t^{\prime}\right)\right]\right\} \\
& \frac{\partial}{\partial t^{\prime}} D_{2 j}\left(V, t^{\prime}\right)=\frac{1}{\gamma_{c}}\left[\lambda_{a}\left(V, t^{\prime}\right)-\lambda_{b}\left(V, t^{\prime}\right)\right] \delta_{j 0}-[\rho \delta+(2 j) i \delta V] D_{2 j}\left(V, t^{\prime}\right) \\
& -i \rho \delta\left[\left(A_{r}\left(t^{\prime}\right) P_{i, 2 j-1}\left(V, t^{\prime}\right)-A_{i}\left(t^{\prime}\right) P_{r, 2 j-1}\left(V, t^{\prime}\right)\right)\right. \\
& \left.-A_{r}\left(t^{\prime}\right) P_{i, 2 j+1}\left(V, t^{\prime}\right)+A_{i}\left(t^{\prime}\right) P_{r, 2 j+1}\left(V, t^{\prime}\right)\right] \\
& \frac{d}{d t^{\prime}} A_{r}\left(t^{\prime}\right)=-\left[A_{r}\left(t^{\prime}\right)+\delta\left(y-y_{0}\right) A_{i}\left(t^{\prime}\right)-\int_{-\infty}^{\infty} P_{i, 1 i}\left(V, t^{\prime}\right) d V\right] \\
& \frac{d}{d t^{\prime}} A_{i}\left(t^{\prime}\right)=-\left[A_{i}\left(t^{\prime}\right)-\delta\left(y-y_{0}\right) A_{r}\left(t^{\prime}\right)+\int_{-\infty}^{\infty} P_{r, 1 i}\left(V, t^{\prime}\right) d V\right]
\end{aligned}
$$

As was suggested in the previous discussion ${ }^{(22)}$, we separate the population 
difference into a broad Gaussian part and a narrow part, $D_{2 j}^{\prime}\left(V, t^{\prime}\right)$ which reflects the localized distortion caused by the saturating laser field as

$$
D_{2 j}\left(V, t^{\prime}\right)=\frac{\varepsilon E_{2 j}\left(t^{\prime}\right)}{\sqrt{\pi}} \exp \left(-\varepsilon^{2} V^{2}\right)+D_{2 j}^{\prime}\left(V, t^{\prime}\right)
$$

And the pumping functions can be written in the form

$$
\begin{aligned}
& \lambda_{a}\left(V, t^{\prime}\right)=\frac{\varepsilon L_{a}\left(t^{\prime}\right)}{\sqrt{\pi}} \exp \left(-\varepsilon^{2} V^{2}\right), \\
& \lambda_{b}\left(V, t^{\prime}\right)=\frac{\varepsilon L_{b}\left(t^{\prime}\right)}{\sqrt{\pi}} \exp \left(-\varepsilon^{2} V^{2}\right),
\end{aligned}
$$

where the background part of the population difference and pumping functions are assumed to posses the Maxwell-Boltzmann velocity distribution in the normalized velocity variable, $V$. By substituting these parameters into Eq. (3.11), the equation may be split into the two parts,

$$
\frac{d}{d t^{\prime}} E_{2 j}\left(t^{\prime}\right)=\frac{1}{\gamma_{c}}\left[L_{a}\left(t^{\prime}\right)-L_{b}\left(t^{\prime}\right)\right] \delta_{j 0}-\delta \rho E_{2 j}\left(t^{\prime}\right)
$$

and $\frac{\partial}{\partial t^{\prime}} D_{2 j}^{\prime}\left(V, t^{\prime}\right)=-[\delta \rho+(2 j) i \delta V] D_{2 j}^{\prime}\left(V, t^{\prime}\right)-\frac{(2 j) i \delta V E_{2 j}\left(t^{\prime}\right)}{\sqrt{\pi}} \exp \left(-\varepsilon^{2} V^{2}\right)$

$$
-i \delta \rho\left[\left(A_{r}\left(t^{\prime}\right) P_{i, 2 j-1}\left(V, t^{\prime}\right)-A_{i}\left(t^{\prime}\right) P_{r, 2 j-1}\left(V, t^{\prime}\right)\right)\right.
$$




$$
\left.-A_{r}\left(t^{\prime}\right) P_{i, 2 j+1}\left(V, t^{\prime}\right)+A_{i}\left(t^{\prime}\right) P_{r, 2 j+1}\left(V, t^{\prime}\right)\right]
$$

\section{THRESHOLD PARAMETER}

At this point it is helpful to introduce the concept of the threshold parameter. This threshold parameter is useful because it provides a direct link between laboratory experiments and theoretical computations. The threshold parameter, $r$ is usually defined as the ratio of the value of actual pump rate of the operating laser to the value of pump rate required for the laser to reach threshold. A relation between pump rates $L_{a}, L_{b}$ and threshold parameter can be expressed as ${ }^{(22)}$

$$
\begin{aligned}
L_{a}= & r L_{a t h} \\
& =\frac{r \sqrt{\pi}}{\varepsilon}\left\{\left[\left(1-\frac{\gamma_{a b}}{\gamma_{b}}\right) \frac{1}{\gamma_{a}}-\frac{L_{b} / L_{a}}{\gamma_{b}}\right] \int_{-\infty}^{\infty} \frac{\exp \left(-\varepsilon^{2} V^{2}\right)}{1+V^{2}} d V\right\}^{-1}
\end{aligned}
$$

where $L_{a t h}$ is the total pump rate to the upper laser level when the laser is at threshold and we also assume that pumping rates, $L_{a}$ and $L_{b}$, are time-independent. From Eq.(3.19), at threshold we have $r=1$. In our study here we have $\gamma_{b}=\gamma_{a}+\gamma_{a b}=\gamma_{d}$, and together with Eq.(3.19) one can write

$$
\begin{aligned}
L_{a} & =\frac{r \sqrt{\pi}}{\varepsilon}\left\{\left[\left(\frac{\gamma_{b}-\gamma_{a b}}{\gamma_{b}}\right) \frac{1}{\gamma_{a}}-\frac{L_{b} / L_{a}}{\gamma_{b}}\right] \int_{-\infty}^{\infty} \frac{\exp \left(-\varepsilon^{2} V^{2}\right)}{1+V^{2}} d V\right\}^{-1}, \\
& =\frac{r \sqrt{\pi}}{\varepsilon}\left\{\left[\left(\frac{\left(\gamma_{a}+\gamma_{a b}\right)-\gamma_{a b}}{\gamma_{b}}\right) \frac{1}{\gamma_{a}}-\frac{L_{b} / L_{a}}{\gamma_{b}}\right] \int_{-\infty}^{\infty} \frac{\exp \left(-\varepsilon^{2} V^{2}\right)}{1+V^{2}} d V\right\}^{-1},
\end{aligned}
$$




$$
\begin{aligned}
& =\frac{r \sqrt{\pi}}{\varepsilon}\left\{\left[\left(\frac{\gamma_{a}}{\gamma_{b}}\right) \frac{1}{\gamma_{a}}-\frac{L_{b} / L_{a}}{\gamma_{b}}\right] \int_{-\infty}^{\infty} \frac{\exp \left(-\varepsilon^{2} V^{2}\right)}{1+V^{2}} d V\right\}^{-1}, \\
& =\frac{r \sqrt{\pi}}{\varepsilon}\left\{\left[\frac{\left(L_{a}-L_{b}\right) / L_{a}}{\gamma_{d}}\right] \int_{-\infty}^{\infty} \frac{\exp \left(-\varepsilon^{2} V^{2}\right)}{1+V^{2}} d V\right\}, \\
& =\frac{r \sqrt{\pi}}{\varepsilon} \times \frac{\gamma_{d} L_{a}}{\left(L_{a}-L_{b}\right)}\left[\int_{-\infty}^{\infty} \frac{\exp \left(-\varepsilon^{2} V^{2}\right)}{1+V^{2}} d V\right]^{-1} .
\end{aligned}
$$

Therefore,

$$
\left(L_{a}-L_{b}\right)=\frac{r \sqrt{\pi}}{\varepsilon} \gamma_{d}\left[\int_{-\infty}^{\infty} \frac{\exp \left(-\varepsilon^{2} V^{2}\right)}{1+V^{2}} d V\right]^{-1}
$$

and $0 \leq L_{b}<L_{a}$. From this one can easily write Eq.(3.17) as

$$
\begin{aligned}
\frac{d}{d t^{\prime}} E_{2 j}\left(t^{\prime}\right) & =\frac{1}{\gamma_{c}}\left[\frac{r \sqrt{\pi}}{\varepsilon} \gamma_{d}\left[\int_{-\infty}^{\infty} \frac{\exp \left(-\varepsilon^{2} V^{2}\right)}{1+V^{2}} d V\right]^{-1}\right] \delta_{j 0}-\delta \rho E_{2 j}\left(t^{\prime}\right) \\
& =\frac{r \sqrt{\pi}}{\varepsilon} \frac{\gamma_{d}}{\gamma_{c}}\left[\int_{-\infty}^{\infty} \frac{\exp \left(-\varepsilon^{2} V^{2}\right)}{1+V^{2}} d V\right]^{-1} \delta_{j 0}-\delta \rho E_{2 j}\left(t^{\prime}\right) \\
& =\delta \rho \frac{r \sqrt{\pi}}{\varepsilon}\left[\int_{-\infty}^{\infty} \frac{\exp \left(-\varepsilon^{2} V^{2}\right)}{1+V^{2}} d V\right]^{-1} \delta_{j 0}-\delta \rho E_{2 j}\left(t^{\prime}\right) \\
& =\delta \rho\left\{\left[\frac{\varepsilon\left(\int_{-\infty}^{\infty} \frac{\exp \left(-\varepsilon^{2} V^{2}\right)}{1+V^{2}} d V\right)}{j} \delta_{j 0}\right]-E_{2 j}\left(t^{\prime}\right)\right\}
\end{aligned}
$$


For numerical computation purposes, it is convenient to introduce a new numbering of the spatial harmonics for the dynamical variables. As suggested earlier ${ }^{(22)}$, the same subscript $k$ can replace both $2 j+1$ in the polarization terms and $2 j$ in the population terms. Equations (3.9), (3.10), (3.18), (3.22) can be replaced by

$$
\begin{aligned}
\frac{\partial}{\partial t^{\prime}} P_{r, k}\left(V, t^{\prime}\right)= & -\delta\left[\{1+(2 k-1) i V\} P_{r, k}\left(V, t^{\prime}\right)+y P_{i, k}\left(V, t^{\prime}\right)\right. \\
& +i A_{i}\left(t^{\prime}\right)\left\{\varepsilon E_{k}\left(t^{\prime}\right) \frac{\exp \left(-\varepsilon^{2} V^{2}\right)}{\sqrt{\pi}}-\varepsilon E_{k+1}\left(t^{\prime}\right) \frac{\exp \left(-\varepsilon^{2} V^{2}\right)}{\sqrt{\pi}}\right. \\
& \left.\left.+D^{\prime}{ }_{k}\left(V, t^{\prime}\right)-D^{\prime}{ }_{k+1}\left(V, t^{\prime}\right)\right\}\right], \\
\frac{\partial}{\partial t^{\prime}} P_{i, k}\left(V, t^{\prime}\right)= & -\delta\left[\{1+(2 k-1) i V\} P_{i, k}\left(V, t^{\prime}\right)-y P_{r, k}\left(V, t^{\prime}\right)\right. \\
& -i A_{r}\left(t^{\prime}\right)\left\{\varepsilon E_{k}\left(t^{\prime}\right) \frac{\exp \left(-\varepsilon^{2} V^{2}\right)}{\sqrt{\pi}}-\varepsilon E_{k+1}\left(t^{\prime}\right) \frac{\exp \left(-\varepsilon^{2} V^{2}\right)}{\sqrt{\pi}}\right. \\
+ & \left.\left.D_{k}^{\prime}\left(V, t^{\prime}\right)-D^{\prime}{ }_{k+1}\left(V, t^{\prime}\right)\right\}\right]
\end{aligned}
$$$$
\frac{\partial}{\partial t^{\prime}} D_{k}^{\prime}\left(V, t^{\prime}\right)=-[\delta \rho+(2 k-2) i \delta V] D^{\prime}{ }_{k}\left(V, t^{\prime}\right)
$$$$
-(2 k-2) i \delta V E_{k}\left(t^{\prime}\right) \frac{\exp \left(-\varepsilon^{2} V^{2}\right)}{\sqrt{\pi}}
$$$$
-i \delta \rho\left[\left(A_{r}\left(t^{\prime}\right) P_{i, k-1}\left(V, t^{\prime}\right)-A_{i}\left(t^{\prime}\right) P_{r, k-1}\left(V, t^{\prime}\right)\right)\right.
$$$$
\left.-A_{r}\left(t^{\prime}\right) P_{i, k}\left(V, t^{\prime}\right)+A_{i}\left(t^{\prime}\right) P_{r, k}\left(V, t^{\prime}\right)\right]
$$ 


$$
\frac{d}{d t^{\prime}} E_{k}\left(t^{\prime}\right)=\delta \rho\left\{\left[\frac{r \sqrt{\pi}}{\varepsilon\left(\int_{-\infty}^{\infty} \frac{\exp \left(-\varepsilon^{2} V^{2}\right)}{1+V^{2}} d V\right)} \delta_{k 1}\right]-E_{k}\left(t^{\prime}\right)\right\}
$$

It was shown ${ }^{(22)}$ that it is not necessary to carry out computations for $k<0$. Eqs.(3.23)-(3.26), and (3.12)-(3.13) are valid for $k>1$, and for $k<1$ the equations can be eliminated by means of the following relations

$$
\begin{gathered}
P_{r,-j}\left(V, t^{\prime}\right)=P_{r, j}^{*}\left(V, t^{\prime}\right), \\
P_{i,-j}\left(V, t^{\prime}\right)=P^{*}{ }_{i, j}\left(V, t^{\prime}\right), \\
D_{-j}\left(V, t^{\prime}\right)=D^{*}{ }_{j}\left(V, t^{\prime}\right) .
\end{gathered}
$$

However, for $k=1$, Eq.(3.25) couples to a more negative value of $k$. In this case we will treat it separately and it proves easier if we work it out from the original equation in $j$ numbering index instead of the equation in $k$ numbering index. We can write Eq.(3.18) for $j=0$ as

$$
\begin{aligned}
\frac{\partial}{\partial t^{\prime}} D_{0}\left(V, t^{\prime}\right)=-\delta \rho D_{0}^{\prime}\left(V, t^{\prime}\right)-i \delta \rho\left[A_{r}\left(t^{\prime}\right) P_{i,-1}\left(V, t^{\prime}\right)\right. \\
\left.-A_{i}\left(t^{\prime}\right) P_{r,-1}\left(V, t^{\prime}\right)-A_{r}\left(t^{\prime}\right) P_{i, 1}\left(V, t^{\prime}\right)+A_{i}\left(t^{\prime}\right) P_{r, 1}\left(V, t^{\prime}\right)\right]
\end{aligned}
$$

From the relations in Eqs.(3.27) and (3.28) we can write 


$$
\begin{aligned}
P_{r,-j}\left(V, t^{\prime}\right) & =\left(P_{r, j}\left(V, t^{\prime}\right)\right)^{*}, \\
& =\left(P_{r, j r}\left(V, t^{\prime}\right)+i P_{r, j i}\right)^{*}=P_{r, j r}\left(V, t^{\prime}\right)-i P_{r, j i}, \\
P_{i,-j}\left(V, t^{\prime}\right) & =\left(P_{i, j}\left(V, t^{\prime}\right)\right)^{*}, \\
& =\left(P_{i, j r}\left(V, t^{\prime}\right)+i P_{i, j i}\right)^{*}=P_{i, j r}\left(V, t^{\prime}\right)-i P_{i, j i},
\end{aligned}
$$

where $P_{r, j r}\left(V, t^{\prime}\right)$ and $P_{r, j i}\left(V, t^{\prime}\right)$ are the real and the imaginary parts of $P_{r, j}\left(V, t^{\prime}\right)$, respectively. The same relation holds for $P_{i, j}\left(V, t^{\prime}\right)$.By separating $P_{r, j}\left(V, t^{\prime}\right)$ and $P_{i, j}\left(V, t^{\prime}\right)$ into their real and imaginary parts as in Eqs.(3.31)-(3.32), one can easily write Eq. (3.30) for $j=0$ as

$$
\begin{aligned}
\frac{\partial}{\partial t^{\prime}} D_{0}^{\prime}\left(V, t^{\prime}\right)= & -\delta \rho D_{0}^{\prime}\left(V, t^{\prime}\right)-i \delta \rho\left\{A_{r}\left(t^{\prime}\right)\left[P_{i, 1}^{*}\left(V, t^{\prime}\right)-P_{i, 1}\left(V, t^{\prime}\right)\right]\right. \\
& \left.+A_{i}\left(t^{\prime}\right)\left[P_{r, 1}^{*}\left(V, t^{\prime}\right)-P_{r, 1}\left(V, t^{\prime}\right)\right]\right\} \\
= & -\delta \rho D_{0}^{\prime}\left(V, t^{\prime}\right)-i \delta \rho\left\{A_{r}\left(t^{\prime}\right)\left(-i 2 P_{i, 1 i}\left(V, t^{\prime}\right)\right)\right. \\
& \left.+A_{i}\left(t^{\prime}\right)\left(-i 2 P_{r, 1 i}\left(V, t^{\prime}\right)\right)\right\} \\
= & -\delta \rho D_{0}^{\prime}\left(V, t^{\prime}\right)-2 \delta \rho\left[A_{r}\left(t^{\prime}\right) P_{i, 1 i}\left(V, t^{\prime}\right)\right. \\
& \left.+A_{i}\left(t^{\prime}\right) P_{r, 1 i}\left(V, t^{\prime}\right)\right]
\end{aligned}
$$

Because the case $j=0$ corresponds to $k=1$ in the $k$ numbering index, from this we can 
write the equation for $D_{k}\left(V, t^{\prime}\right)$ with $k=1$ as

$$
\begin{aligned}
\frac{\partial}{\partial t^{\prime}} D_{1}^{\prime}\left(V, t^{\prime}\right)= & -\delta \rho D^{\prime}{ }_{1}\left(V, t^{\prime}\right)-2 \delta \rho\left[A_{r}\left(t^{\prime}\right) P_{i, 1 i}\left(V, t^{\prime}\right)\right. \\
& \left.+A_{i}\left(t^{\prime}\right) P_{r, 1 i}\left(V, t^{\prime}\right)\right]
\end{aligned}
$$

\section{INITIAL CONDITIONS}

Equations (3.12)-(3.13), (3.23)-(3.26), and (3.34) form a complete set using $k$ values of unity and larger. One can observe that this set of equations is general enough so that the pulsation behavior of lasers operating away from line center can also be determined.

Before starting numerically solving time dependent solutions of the last set of equations above, it helpful to determine initial values for the dependent variables. This approach is useful in initiating the instability of the locally stable system. Casperson ${ }^{(4)}$ showed that it is possible that one might be able to observe unstable behavior of the system even when the linear stability analysis showed that the steady state solutions are stable. The hard excitation of a steady state solution that is locally stable can cause undamped oscillation even at excitation levels below the instability threshold boundary imposed by linear stability analysis. We will consider these two separate instability threshold boundaries in a later chapter.

A useful method in determining the initial values of dependent variables in the above laser equations is based on the idea of loss switching (or Q Switching). By this method the material is assumed to be pumped to some initial value of population difference which corresponds to a particular value of threshold parameter, while the field is held at 
zero. Then a small value of field is injected to begin the computations. Therefore, at the outset the only nonzero dependent variables are the coefficients of the Gaussian population distribution in Eq.(3.14)

At $t^{\prime}=0$ we have for Eq. (3.14)

$$
D_{2 j}(V, 0)=\frac{\varepsilon E_{2 j}(0)}{\sqrt{\pi}} \exp \left(-\varepsilon^{2} V^{2}\right)
$$

The localized distortion terms $D_{2 j}^{\prime}(V, 0)$ are zero at $t^{\prime}=0$ because there is no field at $t^{\prime}=0$. With saturation effects set to zero one can write the steady state form of Eq. (3.26) as

$$
0=\delta \rho\left\{\left[\frac{r \sqrt{\pi}}{\varepsilon\left(\int_{-\infty}^{\infty} \frac{\exp \left(-\varepsilon^{2} V^{2}\right)}{1+V^{2}} d V\right)} \delta_{k 1}\right]-E_{k}\right\} .
$$

One can easily observe that $E_{k}=0$ for $k \neq 1$ and in the case $k=1$ one can write

$$
E_{1}(V)=\frac{r \sqrt{\pi}}{\varepsilon}\left[\int_{-\infty}^{\infty} \frac{\exp \left(-\varepsilon^{2} V^{2}\right)}{1+V^{2}} d V\right]^{-1},
$$

This is the steady state value of $E_{1}$ at threshold and it is also the initial condition of $E_{1}\left(t^{\prime}\right)$. For $k=1$ one can also solve Eq. (3.26) for $E_{1}\left(t^{\prime}\right)$, according to, 


$$
\frac{d}{d t^{\prime}} E_{1}\left(t^{\prime}\right)+\delta \rho E_{1}\left(t^{\prime}\right)=\delta \rho \frac{r \sqrt{\pi}}{\varepsilon\left[\int_{-\infty}^{\infty} \frac{\exp \left(-\varepsilon^{2} V^{2}\right)}{1+V^{2}} d V\right]}
$$

This is a first order differential equation in the standard form $\frac{d}{d t^{\prime}} y\left(t^{\prime}\right)+P\left(t^{\prime}\right) y\left(t^{\prime}\right)=Q\left(t^{\prime}\right)$ which has a solution as

$$
y\left(t^{\prime}\right)=e^{-\int_{0}^{t^{\prime}} P\left(t^{\prime \prime}\right)}\left[\left(\int_{0}^{t^{\prime}} Q\left(t^{\prime \prime}\right) e^{\int_{0}^{\prime \prime} P\left(t^{\prime \prime \prime}\right) d t^{\prime \prime \prime}} d t^{\prime \prime}\right)+c\right],
$$

where $c$ is an integration constant. Therefore one can write a solution of Eq. (3.39) as

$$
\begin{aligned}
E_{1}\left(t^{\prime}\right) & =e^{-\int_{0}^{t^{\prime}} \delta \rho d t^{\prime \prime}}\left[\left(\int_{0}^{t^{\prime}} \frac{\delta \rho r \sqrt{\pi}}{\varepsilon\left(\int_{-\infty}^{\infty} \frac{\exp \left(-\varepsilon^{2} V^{2}\right)}{1+V^{2}} d V\right)} e^{\int_{0}^{t^{\prime \prime}} \delta \rho d t^{\prime \prime \prime}} d t^{\prime \prime}\right]+c\right] \\
& =e^{-\delta \rho t^{\prime}}\left[\left[\frac{\delta \rho r \sqrt{\pi}}{\varepsilon\left(\int_{-\infty}^{\infty} \frac{\exp \left(-\varepsilon^{2} V^{2}\right)}{1+V^{2}} d V\right)} \int_{0}^{t^{\prime}} e^{\delta \rho t^{\prime \prime}}\right]+c\right], \\
& \left.\left.=e^{-\delta \rho t^{\prime}}\right] \frac{\varepsilon\left(\int_{-\infty}^{\infty} \frac{\exp \left(-\varepsilon^{2} V^{2}\right)}{1+V^{2}} d V\right)}{\left.\left(e^{\delta \rho t^{\prime}}-1\right)+c\right]}\right]
\end{aligned}
$$




$$
=\frac{r \sqrt{\pi}}{\varepsilon\left(\int_{-\infty}^{\infty} \frac{\exp \left(-\varepsilon^{2} V^{2}\right)}{1+V^{2}} d V\right)}\left(1-e^{-\delta \rho t^{\prime}}\right)+c e^{-\delta \rho t^{\prime}},
$$

At $t^{\prime}=0$ we have

$$
E_{1}(0)=\frac{r \sqrt{\pi}}{\varepsilon\left(\int_{-\infty}^{\infty} \frac{\exp \left(-\varepsilon^{2} V^{2}\right)}{1+V^{2}} d V\right)}(1-1)+c=c
$$

and from the initial condition of $E_{1}\left(t^{\prime}\right)$ in (3.37) we had

$$
c=E_{1}=\frac{r \sqrt{\pi}}{\varepsilon\left(\int_{-\infty}^{\infty} \frac{\exp \left(-\varepsilon^{2} V^{2}\right)}{1+V^{2}} d V\right)},
$$

Therefore one gets a solution of Eq. (3.38) as

$$
\begin{gathered}
E_{1}\left(t^{\prime}\right)=\frac{r \sqrt{\pi}}{\varepsilon\left(\int_{-\infty}^{\infty} \frac{\exp \left(-\varepsilon^{2} V^{2}\right)}{1+V^{2}} d V\right)}\left(1-e^{-\delta \rho t^{\prime}}\right) \\
+\frac{r \sqrt{\pi}-}{\varepsilon\left(\int_{-\infty}^{\infty} \frac{\exp \left(-\varepsilon^{2} V^{2}\right)}{1+V^{2}} d V\right)} e^{-\delta \rho t^{\prime}}
\end{gathered}
$$




$$
=\frac{r \sqrt{\pi}}{\varepsilon\left(\int_{-\infty}^{\infty} \frac{\exp \left(-\varepsilon^{2} V^{2}\right)}{1+V^{2}} d V\right)}=E_{1} .
$$

which is time-independent. For the case of $k \neq 1$ one can write

$$
\frac{d}{d t^{\prime}} E_{k}\left(t^{\prime}\right)+\delta \rho E_{k}\left(t^{\prime}\right)=0
$$

By method of separation of variables one can solve for $E_{k}\left(t^{\prime}\right)$ as

$$
\begin{aligned}
& \frac{d E_{k}\left(t^{\prime}\right)}{E_{k}\left(t^{\prime}\right)}=-\delta \rho d t^{\prime}, \\
& \ln \left|E_{k}\left(t^{\prime}\right)\right|=-\delta \rho t^{\prime}+c_{1}, \\
& E_{k}\left(t^{\prime}\right)=C e^{-\delta \rho t^{\prime}} .
\end{aligned}
$$

where " In" is the natural logarithmic function and $C=e^{c_{1}}$ is the integration constant. From (3.36) we already knew that $E_{k}(0)=0$ for $k \neq 1$ so we have for Eq. (3.47)

$$
E_{k}(0)=0=C,
$$

The final result for the case $k \neq 1$ is 


$$
E_{k}\left(t^{\prime}\right)=0
$$

for $t^{\prime} \geq 0$. Typically the dependent variables in Eqs. (3.12)-(3.13), (3.23)-(3.26), and (3.34) are initially set to zero, and our derivation above yields $E_{1}(0)=E_{1}$ in Eq. (3.43) as the only nonzero dependent variables at the starting point of the computation.

\section{STRONGLY HOMOGENEOUSLY BROADENED LASER EQUATIONS}

In the strongly homogeneous limit, Eqs. (3.12)-(3.13), (3.23)-(3.26), and (3.34) can be simplified further. The laser equations for this limit are obtained by integrating Eqs. (3.12)-(3.13), (3.23)-(3.26), and (3.34) over velocity while remembering that $V$ is small. Eq. (3.26) has a solution as shown in Eqs. (3.37) and (3.49). The strongly homogeneous limit of Eq. (3.37) is obtained by integrating the equation over the velocity variable, $V$ in the limit as $\varepsilon$ goes to infinite and $V$ goes to zero. That is, from Eq. (3.37),

$$
\begin{aligned}
E_{1}=\lim _{V \rightarrow 0, \varepsilon \rightarrow \infty} E_{1}(V)=\lim _{V \rightarrow 0, \varepsilon \rightarrow \infty}\left(\frac{r \sqrt{\pi}}{\varepsilon}\left[\int_{-\infty}^{\infty} \frac{\exp \left(-\varepsilon^{2} V^{2}\right)}{1+V^{2}} d V\right]^{-1}\right), \\
=\frac{r \sqrt{\pi}}{\varepsilon}\left(\lim _{V \rightarrow 0, \varepsilon \rightarrow \infty}\left[\int_{-\infty}^{\infty} \frac{\exp \left(-\varepsilon^{2} V^{2}\right)}{1+V^{2}} d V\right]^{-1}\right), \\
=\frac{r \sqrt{\pi}}{\varepsilon}\left(\frac{\varepsilon}{\sqrt{\pi}}\right)=r .
\end{aligned}
$$

Next, we introduce new parameters

$$
P_{r, k}\left(t^{\prime}\right)=\int_{-\infty}^{\infty} P_{r, k}\left(V, t^{\prime}\right) d V, P_{i, k}\left(t^{\prime}\right)=\int_{-\infty}^{\infty} P_{i, k}\left(V, t^{\prime}\right) d V,
$$

and

$$
D_{k}^{\prime}\left(t^{\prime}\right)=\int_{-\infty}^{\infty} D_{k}^{\prime}\left(V, t^{\prime}\right) d V
$$


Eqs. (3.12)-(3.13), (3.23)-(3.25), and (3.34) can be rewritten as

$$
\begin{aligned}
\frac{\partial}{\partial t^{\prime}} P_{r, k}\left(t^{\prime}\right)= & -\delta\left[P_{r, k}\left(t^{\prime}\right)+y P_{i, k}\left(t^{\prime}\right)+i A_{i}\left(t^{\prime}\right)\right. \\
& \left.\left(E_{1} \delta_{k 1} D_{k}^{\prime}\left(t^{\prime}\right)-D_{k+1}\left(t^{\prime}\right)\right)\right] \\
\frac{\partial}{\partial t^{\prime}} P_{i, k}\left(t^{\prime}\right)= & -\delta\left[P_{i, k}\left(t^{\prime}\right)-y P_{r, k}\left(t^{\prime}\right)-i A_{i}\left(t^{\prime}\right)\right. \\
& \left.\left(E_{1} \delta_{k 1} D_{k}^{\prime}\left(t^{\prime}\right)-D_{k+1}\left(t^{\prime}\right)\right)\right] \\
& \left.-A_{r}\left(t^{\prime}\right) P_{i, k}\left(t^{\prime}\right)+A_{i}\left(t^{\prime}\right) P_{r, k}\left(t^{\prime}\right)\right] \\
\frac{\partial}{\partial t^{\prime}} D_{k}^{\prime}\left(t^{\prime}\right)= & -\delta \rho D_{k}^{\prime}\left(t^{\prime}\right)-i \delta \rho\left[A_{r}\left(t^{\prime}\right) P_{i, k-1}\left(t^{\prime}\right)-A_{i}\left(t^{\prime}\right) P_{r, k-1}\left(t^{\prime}\right)\right. \\
\frac{\partial}{\partial t^{\prime}} D_{1}^{\prime}\left(V, t^{\prime}\right)= & -\delta \rho D_{1}^{\prime}\left(V, t^{\prime}\right)-2 \delta \rho\left[A_{r}\left(t^{\prime}\right) P_{i, 1 i}\left(V, t^{\prime}\right)\right. \\
& \left.+A_{i}\left(t^{\prime}\right) P_{r, 1 i}\left(V, t^{\prime}\right)\right] \\
\frac{d}{d t^{\prime}} A_{r}\left(t^{\prime}\right)=- & {\left[A_{r}\left(t^{\prime}\right)-\delta\left(y-y_{0}\right) A_{i}\left(t^{\prime}\right)-P_{i, 1 i}\left(t^{\prime}\right)\right] } \\
\frac{d}{d t^{\prime}} A_{i}\left(t^{\prime}\right)=- & {\left[A_{i}\left(t^{\prime}\right)-\delta\left(y-y_{0}\right) A_{r}\left(t^{\prime}\right)+P_{r, 1 i}\left(t^{\prime}\right)\right] } \\
E_{1}\left(t^{\prime}\right)=r, \quad & (k>1) \\
&
\end{aligned}
$$

Eqs. (3.51)-(3.57) have been programmed, by considering the case of $k=1$ and $k \neq 1$ separately, and numerically solved to study the stability criteria of strongly homogeneously broadened standing-wave laser oscillators. The numerical calculation results have proved useful especially in determining the type 2 stability threshold and for lasers that operate at off-line center which we will discuss in a later chapter. 


\section{CHAPTER IV}

\section{STABILITY ANALYSIS}

The stability criteria curves are of substantial practical important. They helps one to easily determine qualitatively the characteristics of the output that a laser with a particular set of operating parameters will produce. In this chapter the methods of finding these stability criteria curves will be studied. As previously studied ${ }^{(4)}$, there are two types of stability criteria that are of special interest and must be distinguished. The first type or "type 1 stability" is the so-called "perturbation stability". Its concerns the smallest value of the threshold parameter for which infinitesimal perturbations of the steady state solutions will increase with time. Qualitatively, what usually is done in this case is, after solving for a steady state solution of a parameter, one introduces a small value of disturbance (perturbation) into that steady state solution and calculates the time dependent value for that parameter. If the perturbation that one introduces does not grow with time, one says that the steady state is stable. But if the perturbation grows with time, one says that the steady state is unstable. In this study we will use the method of "Linear Stability Analysis" to determine this type 1 stability of laser systems.

The second type or "type 2 stability" corresponds to lasers that are initially pulsing, and the threshold parameter is smoothly reduced until the pulsations stop. In this chapter we will apply the model that we have from previous sections to determine these two different types of stability criteria particularly in the limit of strongly homogeneous line broadening. We will study the effects of line center and off line center operations. 


\section{LINEAR STABILITY ANALYSIS}

In determining the type 1 stability curves, we will use the method of linear stability analysis. The method of linear stability analysis is a useful method in the study of dynamical systems that can be used to test whether the steady state solutions of a linear system under consideration are stable when subjected to an infinitesimal perturbation. In simple terms, the method deals with the expansion (linearization) of the unknown (linear or non-linear) solutions of a problem in the small neighborhood of a steady state solution as a sum of that steady state solution plus an infinitesimal perturbation. The linearized equations, which are usually a set of coupled first order differential equations, are then solved by assumed solutions in the exponential form with a complex rate constant. By substituting these assumed solutions into the dynamical equations one can obtain an algebraic equation of this complex rate constant. By solving for solutions of this complex rate constant one can determine whether the steady state solution is stable. The criterion used here is that if all solutions of the complex rate constant equation have negative real part, the steady state solution is stable but if any one of the solutions has positive real part the steady state solution is unstable.

From Eqs.(2.17)-(2.22) if we set $\Gamma_{a}=\Gamma_{b}=0$, according to [29], we have

$$
\begin{aligned}
\left(\frac{\partial}{\partial t}+v \frac{\partial}{\partial z}\right) P_{r}\left(v, \omega_{\alpha}, z, t\right)= & -\left(\omega-\omega_{\alpha}\right) P_{i}\left(v, \omega_{\alpha}, z, t\right)-\gamma P_{r}\left(v, \omega_{\alpha}, z, t\right) \\
& +\frac{\mu^{2}}{\hbar} \sin (k z) E_{i}(t) D\left(v, \omega_{\alpha}, z, t\right), \\
\left(\frac{\partial}{\partial t}+v \frac{\partial}{\partial z}\right) P_{i}\left(v, \omega_{\alpha}, z, t\right)= & \left(\omega-\omega_{\alpha}\right) P_{r}\left(v, \omega_{\alpha}, z, t\right)-\gamma P_{i}\left(v, \omega_{\alpha}, z, t\right)
\end{aligned}
$$




$$
\begin{aligned}
& -\frac{\mu^{2}}{\hbar} \sin (k z) E_{r}(t) D\left(v, \omega_{\alpha}, z, t\right), \\
& \left(\frac{\partial}{\partial t}+v \frac{\partial}{\partial z}\right) D\left(v, \omega_{\alpha}, z, t\right)=\lambda_{a}\left(v, \omega_{\alpha}, z, t\right)-\lambda_{b}\left(v, \omega_{\alpha}, z, t\right) \\
& -\frac{\gamma_{a}+\gamma_{a b}+\gamma_{b}}{2} D\left(v, \omega_{\alpha}, z, t\right)-\frac{\gamma_{a}+\gamma_{a b}-\gamma_{b}}{2} M\left(v, \omega_{\alpha}, z, t\right) \\
& +\frac{\sin (k z)}{\hbar}\left[E_{r}(t) P_{i}\left(v, \omega_{\alpha}, z, t\right)-E_{i}(t) P_{r}\left(v, \omega_{\alpha}, z, t\right)\right], \\
& \left(\frac{\partial}{\partial t}+v \frac{\partial}{\partial z}\right) M\left(v, \omega_{\alpha}, z, t\right)=\lambda_{a}\left(v, \omega_{\alpha}, z, t\right)+\lambda_{b}\left(v, \omega_{\alpha}, z, t\right) \\
& -\frac{\gamma_{a}-\gamma_{a b}-\gamma_{b}}{2} D\left(\nu, \omega_{\alpha}, z, t\right)-\frac{\gamma_{a}-\gamma_{a b}+\gamma_{b}}{2} M\left(v, \omega_{\alpha}, z, t\right), \\
& \frac{d}{d t} E_{r}(t)=-\frac{E_{r}(t)}{2 t_{c}}-(\omega-\Omega) E_{i}(t) \\
& -\frac{\omega_{0}}{\varepsilon_{1} L} \int_{0}^{\infty} \int_{-\infty}^{\infty} \int_{0}^{l} \sin (k z) P_{i}\left(v, \omega_{\alpha}, z, t\right) d z d v d \omega_{\alpha} \\
& \frac{d}{d t} E_{i}(t)=-\frac{E_{i}(t)}{2 t_{c}}+(\omega-\Omega) E_{r}(t) \\
& +\frac{\omega_{0}}{\varepsilon_{1} L} \int_{0}^{\infty} \int_{-\infty}^{\infty} \int_{0}^{l} \sin (k z) P_{r}\left(v, \omega_{\alpha}, z, t\right) d z d v d \omega_{\alpha}
\end{aligned}
$$

The assumption $\Gamma_{a}=\Gamma_{b}=0$ implies that there are no strong velocity changing collisions between gas atoms. This exclusion of the strong velocity collision does not affect the qualitative behavior of the laser system under consideration ${ }^{(22)}$. On the other hand, as pointed out in chapter III, if one wants quantitative agreement between the theoretical and experimental results, this strong velocity collision effect must be retained. 


\section{HOMOGENEOUS BROADENING}

In a medium with homogeneous line broadening, all atoms or molecules of the laser medium interact with the electromagnetic (laser) field in the cavity in the same manner. The response of all atoms, as a whole, can be represented by the response from a single atom member of the laser medium. In gas lasers, especially the high pressure type, the gas atoms or molecules move very rapidly due to thermal or Brownian motion and collide with other atoms in a random manner or with the walls of the laser cavity. These collisions are elastic in the sense that there is no energy loss in the collisions. The collisions, on the other hand, scramble and randomize the phases of oscillations of the colliding atoms or molecules. This dephasing process makes the response of the system to the driving field the same, on average, as that of the individual atoms or molecules of the laser medium and is the major physical process in determining the homogeneously broadened response of gas lasers.

Besides the collision process described, there are also other physical processes which lead to homogeneously broadened response of the collection of laser atoms or molecules; for example, the "lifetime broadening"(35) which is due to the finite lifetimes of the energy levels of the laser atoms. The thermal vibrations of the crystal lattice in solids will modulate the exact distances between laser atoms and nearby host atoms. This will affect the energy level spacings of laser atoms and will modulate the transition frequencies of the laser atoms by small but random amounts with time. This effect is called "phonon broadening"(35). Another example, especially in materials where the laser atoms are sufficiently dense, is the effect of the weak mutual coupling of the oscillating laser atoms which is caused by the local time-varying electromagnetic field generated by a member of the laser atoms is felt by neighboring atoms. This is called "dipolar coupling”(35). This coupling between the individual resonant systems tends to randomize 
and to broaden the response of the whole resonant system. Even if there exists different physical mechanisms which lead to the homogeneous broadened response of the laser transitions, in gas lasers the contributions from these effects are small compared to the dominant part which dues to the physical collisions between atomic members of the gas.

Consider lasers operating in strong homogeneous limit by setting $\omega_{\alpha}=\omega_{0}, v=0$ in Eqs. (4.1)-(4.6), and also introduce a new set of variables ${ }^{(29)}$

$$
\begin{aligned}
& P_{r}(z, t)=\int_{0}^{\infty} \int_{-\infty}^{\infty} P_{r}\left(v, \omega_{\alpha}, z, t\right) d v d \omega_{\alpha}, \\
& P_{i}(z, t)=\int_{0}^{\infty} \int_{-\infty}^{\infty} P_{i}\left(v, \omega_{\alpha}, z, t\right) d v d \omega_{\alpha}, \\
& D(z, t)=\int_{0}^{\infty} \int_{-\infty}^{\infty} D\left(v, \omega_{\alpha}, z, t\right) d v d \omega_{\alpha} \\
& M(z, t)=\int_{0}^{\infty} \int_{-\infty}^{\infty} M\left(v, \omega_{\alpha}, z, t\right) d v d \omega_{\alpha} \\
& \lambda_{a}(z, t)=\int_{0}^{\infty} \int_{-\infty}^{\infty} \lambda_{a}\left(v, \omega_{\alpha}, z, t\right) d v d \omega_{\alpha} \\
& \lambda_{b}(z, t)=\int_{0}^{\infty} \int_{-\infty}^{\infty} \lambda_{b}\left(v, \omega_{\alpha}, z, t\right) d v d \omega_{\alpha} .
\end{aligned}
$$

The condition $\omega_{\alpha}=\omega_{0}$ means that all laser atoms have the same resonant frequency, and $v=0$ means that the average velocity of the laser atoms is zero and we do not need to consider the Doppler broadening effect. The Doppler broadening effect leads to the inhomogeneous broadening response in some types of gas lasers. The velocity integrals that appear in Eqs. (4.7)-(4.12) are integrated over all possible velocities of the moving atoms.

Next we assume a simplified energy-level model, line center operation, and 
consider the empty cavity mode frequency to also be at line center. These conditions can be mathematically represented as

$$
\begin{gathered}
\gamma_{b}=\gamma_{a}+\gamma_{a b}=\gamma_{d}, \\
\omega=\omega_{0}, \\
\Omega=\omega_{0},
\end{gathered}
$$

respectively, where $\gamma_{d}$ is the population difference decay rate as stated before, $\omega$ is the assumed lasing frequency, $\Omega$ is the empty cavity resonant frequency, and $\omega_{0}$ is the resonant frequency of the laser atoms. Equations (4.1)-(4.6) can be rewritten as ${ }^{(29)}$

$$
\begin{aligned}
\frac{\partial}{\partial t} P_{r}(z, t) & =-\gamma P_{r}(z, t)+\frac{\mu^{2}}{\hbar} \sin (k z) E_{i}(t) D(z, t), \\
\frac{\partial}{\partial t} P_{i}(z, t)= & -\gamma P_{i}(z, t)-\frac{\mu^{2}}{\hbar} \sin (k z) E_{r}(t) D(z, t), \\
\frac{\partial}{\partial t} D(z, t)= & \lambda_{d}(z, t)-\gamma_{d} D(z, t) \\
& +\frac{\sin (k z)}{\hbar}\left[E_{r}(t) P_{i}(z, t)-E_{i}(t) P_{r}(z, t)\right], \\
\frac{d}{d t} E_{r}(t)= & -\frac{E_{r}(t)}{2 t_{c}}-\frac{\omega_{0}}{\varepsilon_{1} L} \int_{0}^{l} \sin (k z) P_{i}(z, t) d z, \\
\frac{d}{d t} E_{i}(t)= & -\frac{E_{i}(t)}{2 t_{c}}+\frac{\omega_{0}}{\varepsilon_{1} L} \int_{0}^{l} \sin (k z) P_{r}(z, t) d z,
\end{aligned}
$$


where $\lambda_{d}(z, t)=\lambda_{a}(z, t)-\lambda_{b}(z, t)$. From the last set of equations above one may notice that there is no dynamical equation for the population sum parameter, $M(z, t)$ this is the result of the above assumptions in Eqs. (4.13)-(4.15). The population sum parameter, $M(z, t)$ does not appear in dynamical equations of other parameters, so its dynamical equation is dropped out without loss of information. From this set one can arbitrarily set $P_{r}(z, t)=0, E_{i}(t)=0$, and assume a constant (spatially and temporally) pumping term $\lambda_{d}(z, t)=\lambda_{d}=$ constant. The effect of this is to reduce the number of parameters involved and also reduce the number of dynamic equations. One sees that the equations for the real part of the polarization, $P_{r}(z, t)$ and the imaginary part of the field, $E_{i}(t)$ can be dropped out, and the equation for the population difference, $D(z, t)$ reduces to ${ }^{(29)}$

$$
\frac{\partial}{\partial t} D(z, t)=\lambda_{d}-\gamma_{d} D(z, t)+\frac{\sin (k z)}{\hbar} E_{r}(t) P_{i}(z, t)
$$

It proves helpful to write the laser equations in a normalized form because the normalized set of equations is simpler. A convenient normalization can be obtained from the steady state versions of the equations. At steady state, $\frac{\partial}{\partial t}=0$ one has from Eqs.(4.17), (4.21), and (4.19), respectively

$$
\begin{aligned}
& 0=-\gamma P_{i s}(z)-\frac{\mu^{2}}{\hbar} \sin (k z) E_{r s} D_{s}(z), \\
& 0=\lambda_{d}-\gamma_{d} D_{s}(z)+\frac{\sin (k z)}{\hbar} E_{r s} P_{i s}(z),
\end{aligned}
$$




$$
0=-\frac{E_{r s}}{2 t_{c}}-\frac{\omega_{0}}{\varepsilon_{1} L} \int_{0}^{l} \sin (k z) P_{i s}(z) d z
$$

The subscript $s$ indicates steady state values of the parameters. From Eq. (4.22) one can solve for $P_{i s}(z)$ as

$$
P_{i s}(z)=-\frac{\mu^{2}}{\gamma \hbar} \sin (k z) E_{r s} D_{s}(z)
$$

Substitute the value of $P_{i s}(z)$ into Eq. (4.23) and solve for $D_{s}(z)$ as

$$
\begin{aligned}
& 0=\lambda_{d}-\gamma_{d} D_{s}(z)-\frac{\mu^{2}}{\gamma_{\hbar}^{2}} \sin ^{2}(k z) E_{r s}^{2} D_{s}(z), \\
& =\lambda_{d}-\left(\gamma_{d}+\frac{\mu^{2}}{\gamma \hbar^{2}} \sin ^{2}(k z) E_{r s}^{2}\right) D_{s}(z) \\
& D_{s}(z)=\frac{\left(\lambda_{d} / \gamma_{d}\right)}{1+\frac{\mu^{2}}{\gamma \gamma_{d} \hbar^{2}} \sin ^{2}(k z) E_{r s}^{2}}
\end{aligned}
$$

From Eq. (2.58) we have

$$
A_{r}=\frac{\mu}{2 \hbar}\left[\frac{\gamma_{a}^{\prime}-\gamma_{a b}+\gamma_{b}^{\prime}}{2 \gamma_{a}^{\prime} \gamma_{b}^{\prime}}\right]^{1 / 2} E_{r}
$$




$$
=\frac{\mu}{2 \hbar}\left[\frac{\gamma_{a}-\gamma_{a b}+\gamma_{b}}{2 \gamma \gamma_{a} \gamma_{b}}\right]^{1 / 2} E_{r}
$$

for $\gamma_{a}^{\prime}=\gamma_{a}+\Gamma_{a}, \gamma_{b}^{\prime}=\gamma_{b}+\Gamma_{b}$, and $\Gamma_{a}=\Gamma_{b}=0$. By substituting $\gamma_{b}=\gamma_{a}+\gamma_{a b}=\gamma_{d}$ into Eq. (4.28) one gets

$$
\begin{aligned}
A_{r} & =\frac{\mu}{2 \hbar}\left[\frac{\gamma_{a}-\gamma_{a b}+\gamma_{a}+\gamma_{a b}}{2 \gamma \gamma_{a} \gamma_{d}}\right]^{1 / 2} E_{r} \\
& =\frac{\mu}{2 \hbar}\left[\frac{1}{\gamma \gamma_{d}}\right]^{1 / 2} E_{r}
\end{aligned}
$$

Therefore in Eq. (4.27) we will let $A_{r s}^{2}=\frac{\mu^{2}}{4 \gamma \gamma_{d} \hbar^{2}} E_{r s}^{2}$ in order to get the same normalization factor as in Eq. (4.29). Equation (4.19) can be rewritten in terms of the new normalized field amplitude $A_{r}=\left[\frac{\mu^{2}}{4 \gamma_{d} \hbar^{2}}\right]^{1 / 2} E_{r}$ as $^{(29)}$

$$
\frac{d}{d t} A_{r}(t)=-\gamma_{c}\left[A_{r}(t)+\frac{\omega_{0}}{\varepsilon_{1} L} \frac{\mu}{2 \hbar}\left[\frac{1}{\gamma \gamma_{d}}\right]^{1 / 2} \frac{1}{\gamma_{c}} \int_{0}^{l} \sin (k z) P_{i}(z, t) d z\right]
$$

where $\gamma_{c}=\frac{1}{2 t_{c}}$ is the electric field amplitude decay rate.

Next we introduce a new variable $\zeta$ where ${ }^{(29)}$ 


$$
\begin{aligned}
& \zeta=k z, \quad d \zeta=k d z \\
& \int P_{i}(z, t) d z=\int P_{i}^{\prime}(\zeta, t) d \zeta=\int k P_{i}^{\prime}(\zeta, t) d z
\end{aligned}
$$

and

$$
P_{i}(z, t)=k P_{i}^{\prime}(\zeta, t),
$$

then rewrite Eq. (4.30) as

$$
\frac{d}{d t} A_{r}(t)=-\gamma\left[A_{r}(t)+\frac{\omega_{0}}{\varepsilon_{1} L} \frac{\mu}{2 \hbar}\left[\frac{1}{\gamma \gamma_{d}}\right]^{1 / 2} \frac{1}{\gamma_{c}} \int_{0}^{\zeta_{l}} \sin (\zeta) P^{\prime}{ }_{i}(\zeta, t) d \zeta\right]
$$

where $\zeta_{l}=k l=\left(\frac{\pi}{2}\right)\left(\frac{2 k l}{\pi}\right)$. We define a new variable ${ }^{(29)}$

$$
\begin{aligned}
P_{i}(\zeta, t) & =\frac{\omega_{0}}{\varepsilon_{1} L} \frac{\mu}{2 \hbar}\left[\frac{1}{\gamma_{d}}\right]^{1 / 2} \frac{1}{\gamma_{c}}\left(\frac{2 k l}{\pi}\right) P_{i}^{\prime}(\zeta, t), \\
& =\frac{\omega_{0}}{\varepsilon_{1} L} \frac{\mu}{2 \hbar}\left[\frac{1}{\gamma_{d}}\right]^{1 / 2} \frac{1}{\gamma_{c}}\left(\frac{2 k l}{\pi}\right) \frac{P_{i}(z, t)}{k},
\end{aligned}
$$

this helps us again in rewriting Eq.(4.34) as

$$
\frac{d}{d t} A_{r}(t)=-\gamma_{c}\left[A_{r}(t)+\int_{0}^{\pi / 2} \sin (\zeta) P_{i}(\zeta, t) d \zeta\right]
$$

where the integration $\int_{0}^{\zeta_{l}} \sin (\zeta) P^{\prime}{ }_{i}(\zeta, t) d \zeta$ is approximated by ${ }^{(29)}$ 


$$
\int_{0}^{\zeta_{l}} \sin (\zeta) P_{i}^{\prime}(\zeta, t) d \zeta \cong \frac{2 k l}{\pi} \int_{0}^{\pi / 2} \sin (\zeta) P_{i}^{\prime}(\zeta, t) d \zeta
$$

Equation (4.17) can be rewritten in term of the new parameter $P_{i}(\zeta, t)$ as

$$
\begin{aligned}
\frac{\partial}{\partial t} P_{i}(\zeta, t)= & -\gamma\left\{P_{i}(\zeta, t)+\frac{\mu^{2}}{\gamma \hbar} \sin (\zeta)\left[\frac{2 \hbar}{\mu}\left(\gamma \gamma_{d}\right)^{1 / 2} A_{r}(t)\right]\right. \\
& \left.\times\left[\frac{\omega_{0}}{\varepsilon_{1} L} \frac{\mu}{2 \hbar}\left(\frac{1}{\gamma \gamma_{d}}\right)^{1 / 2} \frac{1}{\gamma_{c}} \frac{2 k l}{\pi} \frac{D(z, t)}{k}\right]\right\}
\end{aligned}
$$

Define a new parameter $D(\zeta, t)$ as ${ }^{(29)}$

$$
\begin{aligned}
D(\zeta, t) & =\frac{\mu^{2}}{\gamma \hbar}\left(\frac{2 \hbar}{\mu}\left(\gamma \gamma_{d}\right)^{1 / 2}\right)\left[\frac{\omega_{0}}{\varepsilon_{1} L} \frac{\mu}{2 \hbar}\left(\frac{1}{\gamma \gamma_{d}}\right)^{1 / 2} \frac{1}{\gamma_{c} k} \frac{2 k l}{\pi}\right] D(z, t), \\
& =\frac{\mu^{2}}{\gamma \hbar} \frac{\omega_{0}}{\varepsilon_{1} L} \frac{1}{\gamma_{c} k} \frac{2 k l}{\pi} D(z, t) .
\end{aligned}
$$

Therefore,

$$
\frac{\partial}{\partial t} P_{i}(\zeta, t)=-\gamma\left[P_{i}(\zeta, t)+\sin (\zeta) A_{r}(t) D(\zeta, t)\right]
$$

and Eq. (4.21) can be rewritten in terms of the new variables $P_{i}(\zeta, t)$ and $D(\zeta, t)$ as 


$$
\begin{aligned}
\frac{\partial}{\partial t} D(z, t)= & -\gamma_{d}\left(D(z, t)-\frac{\lambda_{d}}{\gamma_{d}}-\frac{\sin (\zeta)}{\gamma_{d} \hbar}\left[\frac{2 \hbar}{\mu}\left(\gamma_{d}\right)^{1 / 2} A_{r}(t)\right]\right. \\
& \left.\times\left[\frac{\varepsilon_{1} L}{\omega_{0}} \frac{2 \hbar}{\mu}\left(\gamma \gamma_{d}\right)^{1 / 2} \gamma_{c} k \frac{\pi}{2 k l} P_{i}(\zeta, t)\right]\right\}, \\
\frac{\partial D}{\partial t}(\zeta, t)= & -\gamma_{d}\left\{D(\zeta, t)-\frac{\mu^{2}}{\gamma \hbar} \frac{\omega_{0}}{\varepsilon_{1} L} \frac{1}{\gamma_{c} k} \frac{2 k l}{\pi}\left(\frac{\lambda_{d}}{\gamma_{d}}\right)\right. \\
& -\frac{\mu^{2}}{\gamma^{2}} \frac{\omega_{0}}{\varepsilon_{1} L} \frac{1}{\gamma_{c} k} \frac{2 k l}{\pi} \frac{1}{\gamma_{d} \hbar}\left(\frac{2 \hbar}{\mu}\left(\gamma \gamma_{d}\right)^{1 / 2}\right) \\
& \left.\times\left(\frac{\varepsilon_{1} L}{\omega_{0}} \frac{2 \hbar}{\mu}\left(\gamma_{d}\right)^{1 / 2} \gamma_{c} k\right) \sin (\zeta) A_{r}(t) P_{i}(\zeta, t)\right\} \\
= & -\gamma_{d}\left[D(\zeta, t)-D_{0}-4 \sin (\zeta) A_{r}(t) P_{i}(\zeta, t)\right]
\end{aligned}
$$

where

$$
D_{0}=\frac{\mu^{2}}{\gamma_{h}} \frac{\omega_{0}}{\varepsilon_{1} L} \frac{1}{\gamma_{c} k} \frac{2 k l}{\pi}\left(\frac{\lambda_{d}}{\gamma_{d}}\right)
$$

One can easily observe that $D_{0}$ is the steady state population difference at threshold.

Next if we let $P_{i}(\zeta, t)=2 P^{\prime}{ }_{i}(z, t)$ and $D(\zeta, t)=4 D^{\prime}(\zeta, t)$, Eqs. (4.36), (4.40), and (4.43) can be rewritten in a more symmetrical format ${ }^{(29)}$

$$
\begin{aligned}
& \frac{\partial}{\partial t} P_{i}^{\prime}(\zeta, t)=-\gamma\left[P_{i}^{\prime}(\zeta, t)+2 \sin (\zeta) A_{r}(t) D^{\prime}(\zeta, t)\right] \\
& \frac{\partial}{\partial t} D^{\prime}(\zeta, t)=-\gamma_{d}\left[D^{\prime}(\zeta, t)-D_{0}^{\prime}-2 \sin (\zeta) A_{r}(t) P_{i}^{\prime}(\zeta, t)\right]
\end{aligned}
$$




$$
\frac{d}{d t} A_{r}(t)=-\gamma_{c}\left[A_{r}(t)+2 \int_{0}^{\pi / 2} \sin (\zeta) P_{i}^{\prime}(\zeta, t) d \zeta\right]
$$

where $D_{0}^{\prime}=\frac{D_{0}}{4}$

This last set of equations forms a basis from which the dynamical behavior of the laser system can be studied. Of particular importance for the study of the type 1 stability is the steady state value of the field intensity $I_{s}$ which equals to $A_{r s}^{2}+A_{i s}^{2}$, where $A_{r s}$, and $A_{i s}$ are the real and imaginary parts of the steady state value of the complex field amplitude, $A$, respectively. In our derivation here $A_{i s}=0$, therefore $I_{s}=A_{r s}^{2}$. At steady state, $\frac{\partial}{\partial t}=0$, we have, for Eq. (4.45)

$$
P_{i s}^{\prime}(\zeta)=-2 \sin (\zeta) A_{r s} D_{s}^{\prime}(\zeta)
$$

With this result the steady state value of the population difference from Eq. (4.46) is

$$
\begin{aligned}
{D^{\prime}}_{s}(\zeta) & =D_{0}^{\prime}+2 \sin (\zeta) A_{r s} P_{i s}^{\prime}(\zeta) \\
& =D_{0}^{\prime}-4 \sin ^{2}(\zeta) A_{r s}^{2} D_{s}^{\prime}(\zeta)
\end{aligned}
$$

This equation may be solved to obtain

$$
D_{s}^{\prime}(\zeta)=\frac{D_{0}^{\prime}}{1+4 \sin ^{2}(\zeta) A_{r s}^{2}}
$$

Finally, from Eq.(4.47) the steady state field is

$$
A_{r s}=-2 \int_{0}^{\pi / 2} \sin (\zeta) P_{i s}^{\prime}(\zeta) d \zeta
$$




$$
\begin{aligned}
& =-2 \int_{0}^{\pi / 2} \sin (\zeta)\left[-2 \sin (\zeta) A_{r s} D_{s}^{\prime}(\zeta)\right] d \zeta \\
& =4 A_{r s} \int_{0}^{\pi / 2} \sin ^{2}(\zeta) D_{s}^{\prime}(\zeta) d \zeta
\end{aligned}
$$

Dividing by the field amplitude $A_{r s}$ one obtains

$$
1=4 \int_{0}^{\pi / 2} \sin ^{2}(\zeta) D_{s}^{\prime}(\zeta) d \zeta
$$

At threshold of the laser operation there is no field, $A_{r s}=0$, therefore

$$
D_{s t h}^{\prime}(\zeta)=D_{o}^{\prime}
$$

From Eq. (4.52), considering the steady state condition at threshold one has

$$
\begin{gathered}
1=4 \int_{0}^{\pi / 2} \sin ^{2}(\zeta) D_{0}^{\prime} d \zeta=4 \frac{D_{0}^{\prime}}{2} \frac{\pi}{2}=D_{0}^{\prime} \pi \\
D_{0}^{\prime}=\frac{1}{\pi} .
\end{gathered}
$$

Define the threshold parameter, $r$ where ${ }^{(29)}$

$$
\begin{aligned}
& r=D_{0}^{\prime} \pi \\
& \text { or } \quad D_{0}^{\prime}=\frac{r}{\pi} .
\end{aligned}
$$

Therefore from Eq. (4.50) one can write

$$
D_{s}^{\prime}(\zeta)=\frac{D_{0}^{\prime}}{1+4 \sin ^{2}(\zeta) A_{r s}^{2}}
$$




$$
=\frac{r / \pi}{1+4 \sin ^{2}(\zeta) A_{r s}^{2}}
$$

At laser threshold the value of the threshold parameter equals one. At steady state we have from Eq. (4.52)

$$
\begin{aligned}
1 & =4 \int_{0}^{\pi / 2} \sin ^{2}(\zeta) D_{s}^{\prime}(\zeta) d \zeta, \\
& =4 \int_{0}^{\pi / 2} \sin ^{2}(\zeta) \frac{D_{0}^{\prime}}{1+4 \sin ^{2}(\zeta) A_{r s}^{2}} d \zeta, \\
& =\frac{4 r}{\pi} \int_{0}^{\pi / 2} \frac{\sin ^{2}(\zeta)}{1+4 \sin ^{2}(\zeta) A_{r s}^{2}} d \zeta, \\
& =\frac{4 r}{\pi} \frac{1}{2} \int_{0}^{\pi}\left(\frac{1-\cos (2 \zeta)}{1+4 \sin ^{2}(\zeta) A_{r s}^{2}}\right) d \zeta, \\
& =\frac{2 r}{\pi} \int_{0}^{\pi}\left(\frac{1-\cos (2 \zeta)}{1+4 \sin ^{2}(\zeta) A_{r s}^{2}}\right) d \zeta .
\end{aligned}
$$

The integration in Eq. (4.59) can be carried out by making use of the formula ${ }^{(31)}$

$$
\int_{0}^{\pi / 2} \frac{\cos (2 n x) d x}{1-a^{2} \sin ^{2}(x)}=\frac{(-1)^{n} \pi}{2 \sqrt{1-a^{2}}}\left(\frac{1-\sqrt{1-a^{2}}}{a}\right)^{2 n},
$$

where $n$ is a positive integer and $a^{2}<1$. From Eq. (4.59) 


$$
\begin{aligned}
1 & =\frac{2 r}{\pi} \frac{\pi}{2}\left(\frac{1}{\sqrt{1+4 A_{r s}^{2}}}\right)-\frac{2 r}{\pi}\left(\frac{(-1) \pi}{2 \sqrt{1+4 A_{r s}^{2}}}\right)\left(\frac{\left.1-\sqrt{1+4 A_{r s}^{2}}\right)^{2}}{i\left(2 A_{r s}\right)}\right. \\
& =\frac{r}{\sqrt{1+4 A_{r s}^{2}}}\left[1+\left(\frac{\left.1-\sqrt{1+4 A_{r s}^{2}}\right)^{2}}{i\left(2 A_{r s}\right)}\right],\right. \\
& =\frac{r}{\sqrt{1+4 A_{r s}^{2}}}\left[1+\frac{1-2 \sqrt{1+4 A_{r s}^{2}}+\left(1+4 A_{r s}^{2}\right)}{-4 A_{r s}^{2}}\right], \\
& =\frac{r}{\sqrt{1+4 A_{r s}^{2}}}\left[\frac{-4 A_{r s}^{2}+1-2 \sqrt{1+4 A_{r s}^{2}}+1+4 A_{r s}^{2}}{-4 A_{r s}^{2}}\right], \\
& =\frac{r}{\sqrt{1+4 A_{r s}^{2}}} 2\left[\frac{\left.1-\sqrt{1+4 A_{r s}^{2}}\right],}{-4 A_{r s}^{2}}\right] \\
& =\frac{2 r}{\sqrt{1+4 A_{r s}^{2}}}\left[\frac{\left(1-\sqrt{1+4 A_{r s}^{2}}\right)\left(1+\sqrt{1+4 A_{r s}^{2}}\right)}{\left(-4 A_{r s}^{2}\right)\left(1+\sqrt{1+4 A_{r s}^{2}}\right)}\right], \\
& \left.\frac{1-1-4 A_{r s}^{2}}{\left(1+4 A_{r s}^{2}\right)}\right],
\end{aligned}
$$

Equating Eq.(4.58) and (4.61) we obtain a useful formula ${ }^{(29)}$ 


$$
\begin{aligned}
& \frac{4 r}{\pi} \int_{0}^{\pi / 2} \frac{\sin ^{2}(\zeta)}{1+4 \sin ^{2}(\zeta) A_{r s}^{2}} d \zeta=1=\frac{2 r}{\left(1+4 A_{r s}^{2}\right)+\sqrt{1+4 A_{r s}^{2}}} \\
& \int_{0}^{\pi / 2} \frac{\sin ^{2}(\zeta)}{1+4 \sin ^{2}(\zeta) A_{r s}^{2}} d \zeta=\frac{\pi / 2}{\left(1+4 A_{r s}^{2}\right)+\sqrt{1+4 A_{r s}^{2}}}
\end{aligned}
$$

which we will use it later. From Eq. (4.62) we write

$$
\left(\sqrt{1+4 A_{r s}^{2}}\right)^{2}+\sqrt{1+4 A_{r s}^{2}}-2 r=0
$$

which is a quadratic equation in the parameter $\sqrt{1+4 A^{2}}$ rs with solutions

$$
\sqrt{1+4 A_{r s}^{2}}=\frac{-1 \pm \sqrt{1+8 r}}{2}
$$

Because the quantity $\sqrt{1+4 A_{r s}^{2}}$ is positive, we choose the solution with the plus sign in the middle or

$$
\sqrt{1+4 A_{r s}^{2}}=\frac{-1+\sqrt{1+8 r}}{2}
$$

From this one can solve for $A_{r s}^{2}$ as follows

$$
\begin{aligned}
1+4 A_{r s}^{2} & =\frac{1}{4}(1-2 \sqrt{1+8 r}+1+8 r)=\frac{1}{2}(1+4 r-\sqrt{1+8 r}), \\
A_{r s}^{2} & =\frac{(4 r-1-\sqrt{1+8 r})}{8} .
\end{aligned}
$$

This value of $A_{r s}^{2}$ is the normalized steady state value of the laser field intensity which is an 
important parameter that will be used to test the quantitative agreement between the analytical and numerical computational results.

\section{TYPE 1 (PERTURBATION) STABILITY}

For the study of type 1 (perturbation) stability based on the method of linear stability analysis we will expand solutions of the dynamic equations governing the behavior of the system in terms of the steady state solutions plus small perturbations. That is

$$
\begin{gathered}
P_{i}^{\prime}(\zeta, t)=P_{i s}^{\prime}(\zeta)+P_{i}^{\prime \prime}(\zeta, t), \\
D^{\prime}(\zeta, t)=D_{s}^{\prime}(\zeta)+D^{\prime \prime}(\zeta, t), \\
A_{r}(t)=A_{r s}+A^{\prime \prime}{ }_{r}(t),
\end{gathered}
$$

where the subscript $s$ indicates the steady state solutions and the double primed quantities represent the infinitesimal perturbations of the corresponding parameters ${ }^{(29)}$. Substituting these linearized parameters into their dynamic equations, Eqs. (4.45)-(4.47), and noting that the time derivative, $\frac{\partial}{\partial t}$ of the steady state solutions is zero, we obtain

$$
\begin{aligned}
& \frac{\partial}{\partial t} P^{\prime \prime}{ }_{i}(\zeta, t)=-\gamma\left(P^{\prime}{ }_{i s}(\zeta)+P^{\prime \prime}{ }_{i}(\zeta, t)+2 \sin (\zeta)\right. \\
& \left.\quad \times\left[A_{r s} D^{\prime}{ }_{s}(\zeta)+A_{r s} D^{\prime \prime}(\zeta, t)+A^{\prime \prime}{ }_{r}(t) D^{\prime}{ }_{s}(\zeta)+A^{\prime \prime}{ }_{r}(t) D^{\prime \prime}(\zeta, t)\right]\right\} \\
& \frac{\partial}{\partial t} D^{\prime \prime}(\zeta, t)=-\gamma_{d}\left\{D^{\prime}{ }_{s}(\zeta)+D^{\prime \prime}(\zeta, t)-D_{0}^{\prime}-2 \sin (\zeta)\right. \\
& \left.\quad \times\left[A_{r s} P_{i s}^{\prime}(\zeta)+A_{r s} P_{i}^{\prime \prime}(\zeta, t)+A^{\prime \prime}{ }_{r}(t) P_{i s}{ }^{\prime}(\zeta)+A^{\prime \prime}{ }_{r}(t) P_{i}^{\prime \prime}(\zeta, t)\right]\right\}
\end{aligned}
$$




$$
\frac{d}{d t} A^{\prime \prime}{ }_{r}(t)=-\gamma_{c}\left[A_{r s}+A^{\prime \prime}{ }_{r}(t)+2 \int_{0}^{\pi / 2} \sin (\zeta)\left(P_{i s(\zeta)}^{\prime}+P_{i}^{\prime \prime}(\zeta, t)\right) d \zeta\right]
$$

The quantities $A^{\prime \prime}{ }_{r}(t) D^{\prime \prime}(\zeta, t), A^{\prime \prime}{ }_{r}(t) P_{i}{ }^{\prime \prime}(\zeta, t)$ can be neglected from this set of equations because their values are second order smaller than other parameters in the same equations. By neglecting these second order small terms, the equations reduced to

$$
\begin{aligned}
& \frac{\partial}{\partial t} P_{i}^{\prime \prime}{ }_{i}(\zeta, t)=-\gamma\left[P_{i}^{\prime \prime}{ }_{i}(\zeta, t)+2 \sin (\zeta)\left(A_{r s} D^{\prime \prime}(\zeta, t)+A^{\prime \prime}{ }_{r}(t) D^{\prime}{ }_{s}(\zeta)\right)\right] \\
& \frac{\partial}{\partial t} D^{\prime \prime}(\zeta, t)=-\gamma_{d}\left[D^{\prime \prime}(\zeta, t)-2 \sin (\zeta)\left(A_{r s} P_{i}^{\prime \prime}(\zeta, t)+A^{\prime \prime}{ }_{r}(t) P_{i s}^{\prime}(\zeta)\right)\right] \\
& \frac{d}{d t} A^{\prime \prime}{ }_{r}(t)=-\gamma_{c}\left[A^{\prime \prime}{ }_{r}(t)+2 \int_{0}^{\pi / 2} \sin (\zeta) P^{\prime \prime}{ }_{i}(\zeta, t) d \zeta\right]
\end{aligned}
$$

This is a set of coupled first order nonlinear differential equations which in general is difficult to solve analytically but one still can always solve it numerically. Of particular interest here, however, is not the value of the solutions themselves (but if one can solve for them, it will be the best), instead it is the qualitative temporal dynamic behaviors of the solutions. In this case we assume solutions of the above equations in the exponential form as ${ }^{(29)}$

$$
\begin{gathered}
P_{i}^{\prime \prime}(\zeta, t)=P^{\prime \prime \prime}(\zeta) \exp (s t), \\
D^{\prime \prime}(\zeta, t)=D^{\prime \prime \prime}(\zeta) \exp (s t), \\
A^{\prime \prime}{ }_{r}(t)=A^{\prime \prime \prime}{ }_{r} \exp (s t),
\end{gathered}
$$


where the temporal behaviors of the solutions are assumed to be absorbed into and determined only by the complex rate constant $s$. With these solutions substituted into Eqs.(4.74)-(4.76) we get

$$
\begin{aligned}
& s P^{\prime \prime \prime}{ }_{i}(\zeta)=-\gamma\left[P^{\prime \prime \prime}{ }_{i}(\zeta)+2 \sin (\zeta)\left(A_{r s} D^{\prime \prime \prime}(\zeta)+A^{\prime \prime \prime}{ }_{r}{ }^{\prime}(\zeta)\right)\right] \\
& s D^{\prime \prime \prime}(\zeta)=-\gamma_{d}\left[D^{\prime \prime \prime}(\zeta)-2 \sin (\zeta)\left(A_{r s} P^{\prime \prime \prime}{ }_{i}(\zeta)+A^{\prime \prime \prime}{ }_{r} P^{\prime}{ }_{i s}(\zeta)\right)\right] \\
& s A^{\prime \prime \prime}{ }_{r}=-\gamma_{c}\left[A^{\prime \prime \prime}{ }_{r}+2 \int_{0}^{\pi / 2} \sin (\zeta) P^{\prime \prime \prime}{ }_{i}(\zeta) d \zeta\right]
\end{aligned}
$$

Rearrange the equations as

$$
\begin{aligned}
& (s+\gamma) P^{\prime \prime \prime}{ }_{i}(\zeta)=-2 \gamma \sin (\zeta)\left[A^{\prime \prime \prime}{ }_{r} D_{s}^{\prime}(\zeta)+A_{r s} D^{\prime \prime \prime}(\zeta)\right] \\
& \left(s+\gamma_{d}\right) D^{\prime \prime \prime}(\zeta)=2 \gamma_{d} \sin (\zeta)\left[A^{\prime \prime \prime}{ }_{r} P_{i s}^{\prime}(\zeta)+A_{r s} P^{\prime \prime \prime}{ }_{i}(\zeta)\right] \\
& \left(s+\gamma_{c}\right) A^{\prime \prime \prime}{ }_{r}=-2 \gamma_{c} \int_{0}^{\pi / 2} \sin (\zeta) P^{\prime \prime \prime}{ }_{i}(\zeta) d \zeta
\end{aligned}
$$

and solve for $P^{\prime \prime \prime}{ }_{i}(\zeta), D^{\prime \prime \prime}(\zeta)$, and $A^{\prime \prime \prime}{ }_{r}$ as

$$
\begin{aligned}
P^{\prime \prime \prime}{ }_{i}(\zeta)= & -\frac{2 \gamma \sin (\zeta)}{(s+\gamma)}\left[A^{\prime \prime \prime}{ }_{r}\left(\frac{r / \pi}{1+4 \sin ^{2}(\zeta) A_{r s}^{2}}\right)+A_{r s} D^{\prime \prime \prime}(\zeta)\right] \\
D^{\prime \prime \prime}(\zeta)= & \frac{2 \gamma_{d} \sin (\zeta)}{\left(s+\gamma_{d}\right)}\left[A^{\prime \prime \prime}{ }_{r}\left(-2 \sin (\zeta) A_{r s}\left(\frac{r / \pi}{1+4 \sin ^{2}(\zeta) A_{r s}^{2}}\right)\right)\right. \\
& \left.+A_{r s} P^{\prime \prime \prime}{ }_{i}(\zeta)\right]
\end{aligned}
$$




$$
\begin{aligned}
& =\frac{2 \gamma_{d} \sin (\zeta)}{\left(s+\gamma_{d}\right)}\left[-\frac{A^{\prime \prime \prime}{ }_{r} \sin (\zeta) A_{r s}\left(\frac{2 r}{\pi}\right)}{1+4 \sin ^{2}(\zeta) A_{r s}^{2}}+A_{r s} P^{\prime \prime}{ }_{i}(\zeta)\right], \\
A^{\prime \prime \prime}{ }_{r} & =-\frac{2 \gamma_{c}}{\left(s+\gamma_{c}\right)} \int_{0}^{\pi / 2} \sin (\zeta) P^{\prime \prime \prime}{ }_{i}(\zeta) d \zeta .
\end{aligned}
$$

where we also use Eq.(4.48), and (4.58) to substitute for parameters $D^{\prime}{ }_{s}(\zeta)$ and $P^{\prime}{ }_{i s}(\zeta)$.

Next we eliminate $D^{\prime \prime \prime}(\zeta)$ from the last set of equations by substituting $D^{\prime \prime}(\zeta)$ from Eq. (4.87) into Eq. (4.86):

$$
\begin{aligned}
& P^{\prime \prime \prime}{ }_{i}(\zeta)=-\frac{2 \gamma \sin (\zeta)}{(s+\gamma)}\left\{\frac{A^{\prime \prime \prime}{ }_{r}(r / \pi)}{1+4 \sin ^{2}(\zeta) A_{r s}^{2}}+\frac{2 \gamma_{d} A_{r s} \sin (\zeta)}{\left(s+\gamma_{d}\right)}\right. \\
& \left.\times\left[-\frac{A^{\prime \prime \prime}{ }_{r} \sin (\zeta) A_{r s}\left(\frac{2 r}{\pi}\right)}{1+4 \sin ^{2}(\zeta) A_{r s}^{2}}+A_{r s} P^{\prime \prime \prime}{ }_{i}(\zeta)\right]\right\} \\
& {\left[1+\frac{4 \gamma \gamma_{d} A_{r s}^{2} \sin ^{2}(\zeta)}{(s+\gamma)\left(s+\gamma_{d}\right)}\right] P^{\prime \prime \prime}{ }_{i}(\zeta)=-\frac{2 \gamma \sin (\zeta) A^{\prime \prime \prime}{ }_{r}(r / \pi)}{(s+\gamma)\left(1+4 \sin ^{2}(\zeta) A_{r s}^{2}\right)}} \\
& \times\left[1-\frac{4 \gamma_{d} A_{r s}^{2} \sin ^{2}(\zeta)}{\left(s+\gamma_{d}\right)}\right] \\
& P^{\prime \prime \prime}{ }_{i}(\zeta)=-\frac{\left[\frac{2 \gamma \sin (\zeta) A^{\prime \prime \prime}{ }_{r}(r / \pi)}{(s+\gamma)\left(1+4 \sin ^{2}(\zeta) A_{r s}^{2}\right)}\right]\left[1-\frac{4 \gamma_{d} A_{r s}^{2} \sin ^{2}(\zeta)}{\left(s+\gamma_{d}\right)}\right]}{\left[1+\frac{4 \gamma \gamma_{d} A_{r s}^{2} \sin ^{2}(\zeta)}{(s+\gamma)\left(s+\gamma_{d}\right)}\right]}
\end{aligned}
$$


Then substitute this value of $P^{\prime \prime \prime}{ }_{i}(z)$ into Eq. (4.88) and pull $A^{\prime \prime \prime}{ }_{r}$ out of the integral $\operatorname{sign}^{(29)}$ :

$$
\begin{aligned}
A^{\prime \prime \prime}{ }_{r} & =\frac{4 \gamma_{c} A^{\prime \prime \prime}{ }_{r}(r / \pi)}{(s+\gamma)\left(s+\gamma_{c}\right)} \int_{0}^{\pi / 2} \frac{\sin ^{2}(\zeta)\left[1-\frac{4 \gamma_{d} A_{r s}^{2} \sin ^{2}(\zeta)}{\left(s+\gamma_{d}\right)}\right]}{\left(1+4 \sin ^{2}(\zeta) A_{r s}^{2}\right)\left[1+\frac{4 \gamma \gamma_{d} A_{r s}^{2} \sin ^{2}(\zeta)}{(s+\gamma)\left(s+\gamma_{d}\right)}\right]} d \zeta, \\
1 & =\frac{4 \gamma_{c}(r / \pi)}{(s+\gamma)\left(s+\gamma_{c}\right)} \int_{0}^{\pi / 2} \frac{\sin ^{2}(\zeta)\left[1-\frac{4 \gamma_{d} A_{r s}^{2} \sin ^{2}(\zeta)}{\left(s+\gamma_{d}\right)}\right]}{\left(1+4 \sin ^{2}(\zeta) A_{r s}^{2}\right)\left[1+\frac{4 \gamma_{d} A_{r s}^{2} \sin ^{2}(\zeta)}{(s+\gamma)\left(s+\gamma_{d}\right)}\right]} d \zeta, \\
& =I_{1}+I_{2},
\end{aligned}
$$

where

$$
\begin{gathered}
I_{1}=C \int_{0}^{\pi / 2} \frac{\sin ^{2}(\zeta)}{\left(1+4 \sin ^{2}(\zeta) A_{r s}^{2}\right)\left[1+\frac{4 \gamma \gamma_{d} A_{r s}^{2} \sin ^{2}(\zeta)}{(s+\gamma)\left(s+\gamma_{d}\right)}\right]} d \zeta, \\
I_{2}=C\left[-\frac{4 \gamma_{d} A_{r s}^{2}}{\left(s+\gamma_{d}\right)}\right] \int_{0}^{\pi / 2} \frac{\left[\sin ^{2}(\zeta)\right]^{2}}{\left(1+4 \sin ^{2}(\zeta) A_{r s}^{2}\right)\left[1+\frac{4 \gamma \gamma_{d} A_{r s}^{2} \sin ^{2}(\zeta)}{(s+\gamma)\left(s+\gamma_{d}\right)}\right]} d \zeta, \\
C=\frac{4 \gamma_{c}(r / \pi)}{(s+\gamma)\left(s+\gamma_{c}\right)} .
\end{gathered}
$$


$\frac{x}{(1+a x)(1+b x)}$, and $\frac{x^{2}}{(1+a x)(1+b x)}$, which can be written in terms of partial fractions as ${ }^{(29)}$

$$
\begin{aligned}
& \frac{x}{(1+a x)(1+b x)}=\frac{c}{(1+a x)}+\frac{d}{(1+b x)} \\
& =\frac{c+c b x+d+a d x}{(1+a x)(1+b x)} \\
& =\frac{(c+d)+(a d+c b) x}{(1+a x)(1+b x)}
\end{aligned}
$$

then $c+d=0$, and $(a d+c b)=1$, which can be solved for $c$, and $d$ as

$$
\begin{aligned}
& c=-d, \\
& c=\frac{1}{(b-a)},
\end{aligned}
$$

and

$$
d=-\frac{1}{(b-a)}
$$

The partial fraction of $\frac{x^{2}}{(1+a x)(1+b x)}$ is

$$
\begin{aligned}
\frac{x^{2}}{(1+a x)(1+b x)} & =x\left[\frac{x}{(1+a x)(1+b x)}\right] \\
& =x\left[\frac{c}{(1+a x)}+\frac{d}{(1+b x)}\right]
\end{aligned}
$$




$$
=\frac{c x}{(1+a x)}+\frac{d x}{(1+b x)} \text {. }
$$

From Eqs. (4.92)-(4.93) we have for $a, b$, and $x$

$$
\begin{aligned}
a & =4 A_{r s}^{2}, \\
b & =\frac{4 \gamma \gamma_{d} A_{r s}^{2}}{(s+\gamma)\left(s+\gamma_{d}\right)}, \\
& =\frac{\gamma \gamma_{d} a}{(s+\gamma)\left(s+\gamma_{d}\right)}, \\
x & =\sin ^{2}(\zeta)
\end{aligned}
$$

We have from Eq. (4.63)

$$
\begin{aligned}
& \int_{0}^{\pi / 2} \frac{\sin ^{2}(\zeta)}{1+4 \sin ^{2}(\zeta) A_{r s}^{2}} d \zeta=\frac{\pi / 2}{\left(1+4 A_{r s}^{2}\right)+\sqrt{1+4 A_{r s}^{2}}} \\
& \int_{0}^{\pi / 2} \frac{\sin ^{2}(\zeta)}{1+a \sin ^{2}(\zeta)} d \zeta=\frac{\pi / 2}{(1+a)+\sqrt{1+a}}
\end{aligned}
$$

and from Eq. (4.61) we have

$$
\int_{0}^{\pi / 2} \frac{d \zeta}{1-a^{2} \sin ^{2}(\zeta)}=\frac{\pi / 2}{\sqrt{1-a^{2}}}
$$


or

$$
\int_{0}^{\pi / 2} \frac{d \zeta}{1+a \sin ^{2}(\zeta)}=\frac{\pi / 2}{\sqrt{1+a}}
$$

Therefore Eqs. (4.92), and (4.93) can be rewritten as

$$
\begin{aligned}
& I_{1}=C\left[c \int_{0}^{\pi / 2} \frac{d \zeta}{1+a \sin ^{2} \zeta}+d \int_{0}^{\pi / 2} \frac{d \zeta}{1+b \sin ^{2} \zeta}\right] \\
& I_{2}=C\left(-\frac{4 \gamma_{d} A_{r s}^{2}}{s+\gamma_{d}}\right)\left[c \int_{0}^{\pi / 2} \frac{\sin ^{2} \zeta d \zeta}{1+a \sin ^{2} \zeta}+d \int_{0}^{\pi / 2} \frac{\sin ^{2} \zeta d \zeta}{1+b \sin ^{2} \zeta}\right]
\end{aligned}
$$

which can be evaluated with the aid of Eqs. (4.104), and (4.106) as

$$
\begin{aligned}
& I_{1}=C \frac{\pi}{2}\left[\frac{c}{\sqrt{1+a}}+\frac{d}{\sqrt{1+b}}\right] \\
& I_{2}=C\left(-\frac{4 \gamma_{d} A_{r s}^{2}}{s+\gamma_{d}}\right) \frac{\pi}{2}\left[\frac{c}{1+a+\sqrt{1+a}}+\frac{d}{1+b+\sqrt{1+b}}\right]
\end{aligned}
$$

From Eqs. (4.97)-(4.98) we also have $c=-d=\frac{1}{(b-a)}$, therefore

$$
I_{1}=C \frac{\pi}{2} \frac{1}{(b-a)}\left[\frac{1}{\sqrt{1+a}}-\frac{1}{\sqrt{1+b}}\right]
$$


and

$$
I_{2}=C\left(-\frac{4 \gamma_{d} A_{r s}^{2}}{s+\gamma_{d}}\right) \frac{\pi}{2} \frac{1}{(b-a)}\left[\frac{1}{1+a+\sqrt{1+a}}-\frac{1}{1+b+\sqrt{1+b}}\right]
$$

Substitute the values of $I_{1}, I_{2}, a, b$, and $C$ from Eqs. (4.94), (4.100), (4.101), and (4.111)(4.112) into Eq. (4.91) we get the result ${ }^{(29)}$

$$
\begin{aligned}
& 1=\left(\frac{4 \gamma_{c}(r / \pi)}{(s+\gamma)\left(s+\gamma_{c}\right)}\right) \frac{\pi}{2} \frac{1}{\left(\frac{\gamma \gamma_{d}\left(4 A_{r s}^{2}\right)}{(s+\gamma)\left(s+\gamma_{d}\right)}-4 A_{r s}^{2}\right)} \\
& \times 1\left(\frac{1}{\sqrt{1+4 A_{r s}^{2}}}-\frac{1}{\sqrt{1+\frac{\gamma \gamma_{d}\left(4 A_{r s}^{2}\right)}{(s+\gamma)\left(s+\gamma_{d}\right)}}}\right)-\left(\frac{4 \gamma_{d} A_{r s}^{2}}{s+\gamma_{d}}\right)\left[\frac{1}{1+4 A_{r s}^{2}+\sqrt{1+4 A_{r s}^{2}}}\right. \\
& \left.\left.-\frac{1}{1+\frac{\gamma \gamma_{d}\left(4 A_{r s}^{2}\right)}{(s+\gamma)\left(s+\gamma_{d}\right)}+\sqrt{1+\frac{\gamma \gamma_{d}\left(4 A_{r s}^{2}\right)}{(s+\gamma)\left(s+\gamma_{d}\right)}}}\right]\right\} \\
& 1=\left(\frac{2 \gamma_{c}{ }^{r}}{(s+\gamma)\left(s+\gamma_{c}\right)}\right) \frac{1}{\left(\frac{\gamma \gamma_{d}\left(4 A_{r s}^{2}\right)}{(s+\gamma)\left(s+\gamma_{d}\right)}-4 A_{r s}^{2}\right)} \\
& \times\left\{\frac{1}{\sqrt{1+4 A_{r s}^{2}}}-\frac{1}{\sqrt{1+\frac{\gamma \gamma_{d}\left(4 A_{r s}^{2}\right)}{(s+\gamma)\left(s+\gamma_{d}\right)}}}\right)-\left(\frac{4 \gamma_{d} A_{r s}^{2}}{s+\gamma_{d}}\right)\left[\frac{1}{\left(1+4 A_{r s}^{2}\right)+\sqrt{1+4 A_{r s}^{2}}}\right.
\end{aligned}
$$




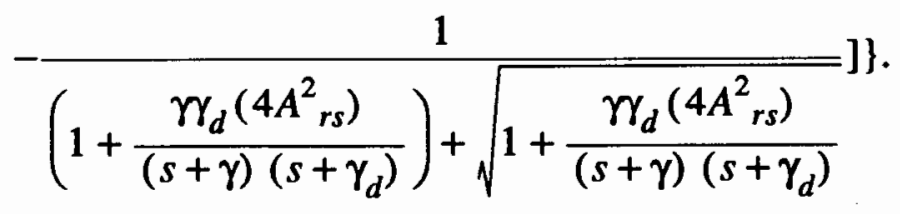

One observes that it is impossible to solve the last equation for the values of $s$ analytically because of the complication of the radicals but one can still solve it numerically. However, Eq. (4.113) still contains too many variables which complicate the form of equation. By introducing the dimensionless parameters $\delta=\gamma / \gamma_{c}$, and $\rho=\gamma_{d} / \gamma$, as in chapter III, one can write Eq. (4.113) as

$$
1=\frac{2 \delta r}{\left(s^{\prime}+1\right)\left(s^{\prime}+\delta\right)} \frac{1}{\left[\frac{\delta^{2} \rho\left(4 A_{r s}^{2}\right)}{\left(s^{\prime}+\delta\right)\left(s^{\prime}+\delta \rho\right)}-4 A_{r s}^{2}\right]}
$$

$$
\begin{gathered}
\quad\left[\frac{1}{\sqrt{1+4 A_{r s}^{2}}}-\frac{1}{\sqrt{1+\frac{\delta^{2} \rho\left(4 A_{r s}^{2}\right)}{\left(s^{\prime}+\delta\right)\left(s^{\prime}+\delta \rho\right)}}}\right]-\left(\frac{4 A_{r s}^{2} \delta \rho}{\left(s^{\prime}+\delta \rho\right)}\right)\left[\frac{1}{\left(1+4 A_{r s}^{2}\right)+\sqrt{1+4 A_{r s}^{2}}}\right. \\
\left.\left.-\frac{1}{\left(1+\frac{\delta^{2} \rho 4 A_{r s}^{2}}{\left(s^{\prime}+\delta\right)\left(s^{\prime}+\delta \rho\right)}\right)+\sqrt{1+\frac{\delta^{2} \rho 4 A_{r s}^{2}}{\left(s^{\prime}+\delta\right)\left(s^{\prime}+\delta \rho\right)}}}\right]\right\}
\end{gathered}
$$

where the new complex rate constant $s^{\prime}=\frac{s}{\gamma_{c}}$. Next we introduce a new set of parameters 


$$
\begin{aligned}
& \mathbf{a}=\left(s^{\prime}+\delta\right)\left(s^{\prime}+\delta \rho\right) \\
& \mathbf{b}=4 A_{r s}^{2} \delta^{2} \rho=\mathbf{c}\left(\delta^{2} \rho\right) \\
& \mathbf{c}=4 A_{r s}^{2}, \\
& \mathbf{f}=\frac{1}{\sqrt{1+4 A_{r s}^{2}}}=\frac{1}{\sqrt{1+\mathbf{c}}}, \\
& \mathbf{e}=\frac{1}{\left(1+4 A_{r s}^{2}\right)+\sqrt{1+4 A_{r s}^{2}}}=\frac{1}{(1+\mathrm{c})+\sqrt{1+\mathrm{c}}}
\end{aligned}
$$

and with these new parameters one can rewrite Eq. (4.114) as

$$
\begin{aligned}
& 1=\frac{2 \delta r}{\left(s^{\prime}+1\right)\left(s^{\prime}+\delta\right)}\left[\frac{1}{\left(\frac{\mathbf{b}}{\mathbf{a}}-\mathbf{c}\right)}\right] \\
& {\left[\left[\mathbf{f}-\frac{1}{\sqrt{1+\frac{\mathbf{b}}{\mathbf{a}}}}\right]-\frac{\mathbf{b}}{\delta\left(s^{\prime}+\delta \rho\right)}\left[\mathrm{e}-\frac{1}{\left(1+\frac{\mathbf{b}}{\mathbf{a}}\right)+\sqrt{1+\frac{\mathbf{b}}{\mathbf{a}}}}\right]\right),} \\
& =\frac{2 \delta r}{\left(s^{\prime}+1\right)\left(s^{\prime}+\delta\right)}\left[\frac{\mathbf{a}}{(\mathbf{b}-\mathbf{a c})}\right] \\
& {\left[\left[\mathbf{f}-\frac{1}{\sqrt{1+\frac{\mathbf{b}}{\mathbf{a}}}}\right]-\frac{\mathbf{b}}{\delta\left(s^{\prime}+\delta \rho\right)}\left[\mathrm{e}-\frac{\left(1+\frac{\mathbf{b}}{\mathbf{a}}\right)-\sqrt{1+\frac{\mathbf{b}}{\mathbf{a}}}}{\left(1+\frac{\mathbf{b}}{\mathbf{a}}\right)^{2}-\left(1+\frac{\mathbf{b}}{\mathbf{a}}\right)}\right]\right) .}
\end{aligned}
$$


$\frac{\mathbf{u}}{\left[\delta \mathbf{b}\left(s^{\prime}+1\right)(\mathbf{b}-\mathbf{a c}) \mathbf{a}^{2}(\mathbf{a}+\mathbf{b})\right]^{2}}=0$,

where

$$
\begin{aligned}
\mathbf{u}= & \left\{\mathbf{a}^{2} \mathbf{b} \delta(\mathbf{a}+\mathbf{b})\left[2 r\left(\mathbf{f} \delta\left(s^{\prime}+\delta \rho\right)+(\mathbf{a}-\mathbf{b e})\right]\right]-\left[\left(s^{\prime}+1\right)(\mathbf{b}-\mathbf{a c})\right]\right\}^{2} \\
& -\left\{2 \delta r \mathbf{a}^{2} \mathbf{b}\left[\delta\left(s^{\prime}+\delta \rho\right)+\mathbf{a}\right]\right\}^{2} \mathbf{a}(\mathbf{a}+\mathbf{b}) .
\end{aligned}
$$

Therefore in order to satisfy Eq. (4.122), the value of $\mathbf{u}$ has to be zero. One sees that the radical equation, Eq. (4.120) has been transformed to a polynomial equation, Eq. (4.122) which can be programmed and solved numerically. If one expands Eq. (4.123), the result will be a polynomial degree $18^{\text {th }}$ in the parameter $s^{\prime}$ which is the normalized complex rate constant. In this study Eq. (4.123) has been programmed and solved by using a mathematical software package called "Mathematica" version 1.2. The solutions of the equation were also substituted back into Eq. (4.120) in order to check their validity because in the process of transforming the radical equation into the polynomial equation above we have introduced some excess roots into the original equation. These excess roots can be filtered out by substituting the solutions of the polynomial equation into the radical equation. If the radical equation is satisfied, that root is the real solution of the original radical equation. On the other hand, if the radical is not satisfied, the root is an excess (or "spurious") root and we discard it from the final result. The results of the calculation will be presented in the next chapter. 


\section{TYPE 2 STABILITY}

For this type of stability criteria, the result is curves in laser parameter space that correspond to the minimum values of threshold parameter with which large amplitude oscillations will not decay with time. This threshold condition corresponds to a laser that initially pulsing. The threshold parameter is then gradually reduced until the pulsation stop. The boundaries are most convenient obtained by direct numerical solution of the laser equations, Eqs. (3.51)-(3.57), for different sets of operating parameters as initial conditions. The time integrations had been performed by using a modified second order Runge-Kutta method $^{(32)}$. The value of the number of spatial harmonics was chosen so that the numerically computed steady state value of laser intensity differs from the analytical result, Eq. (4.67), by less than 1 percent and that the numerical calculations have to show unstable behavior in the unstable area of parameter space which is predicted by type 1 stability criteria, that is above the type 1 stability boundaries. The value of the number of spatial harmonics used in the numerical is 18 . The flow chart of the numerical calculation routine is shown in Figure 1. The computer program used is genaral enough to apply to lasers that are detuned away from line center. The type 2 stability boundaries will be presented in the next chapter.

\section{DETUNING EFFECT}

The next study is to observe the effect of detuning away from line center of the atomic gain profile to stability criteria. The theoretical models obtained in chapter II and III also include this effect in the dynamic equation describing the electric field, Eqs. (2.73)-(2.74), (3.12)-(3.13), and (3.55)-(3.56). In the models there are three different values of frequency, i.e. atomic center transition frequency, $\omega_{0}$, nondispersed (or empty) cavity frequency, $\Omega$, and laser frequency, $\omega$. In the normalized form they are represented 


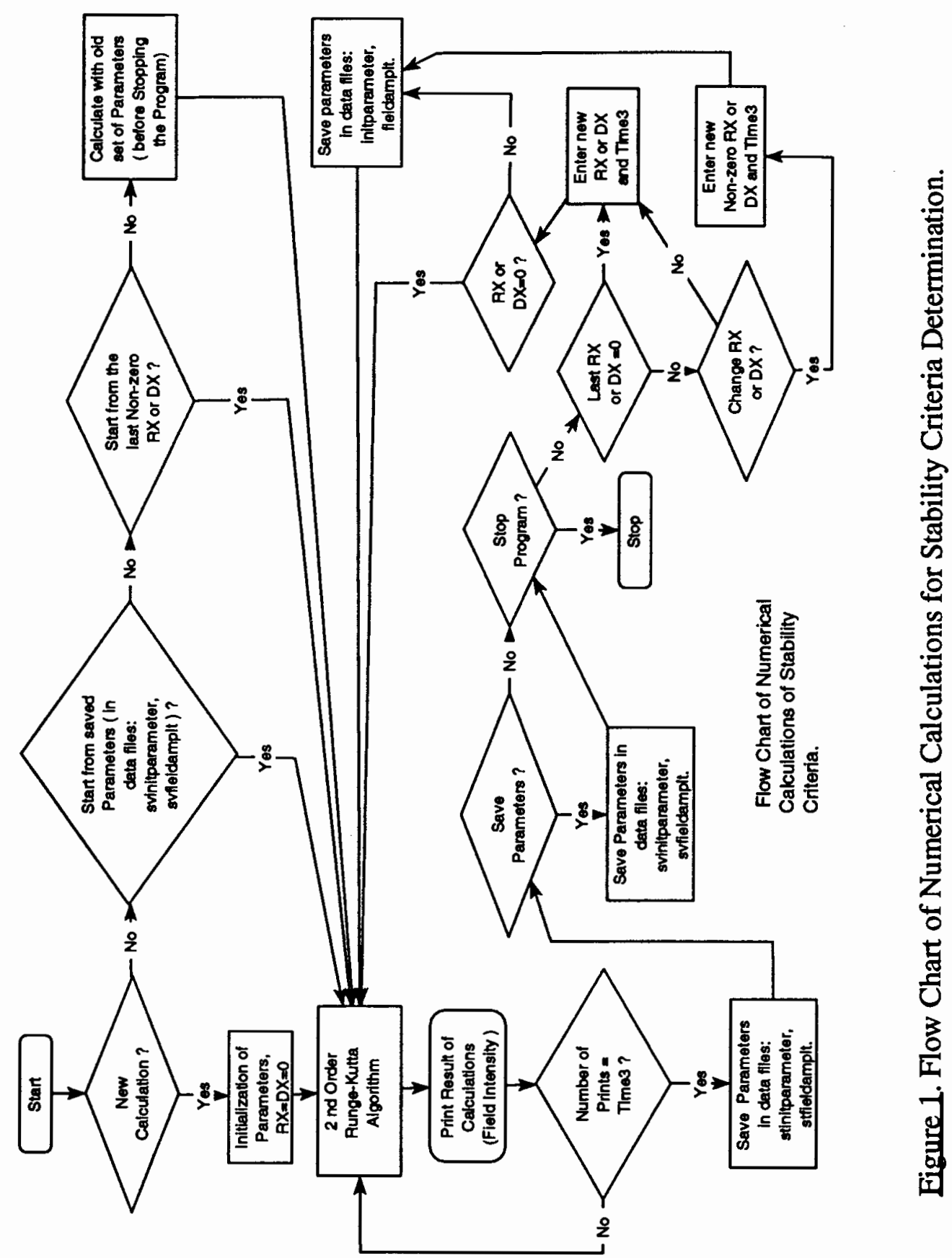


by the parameters $U, y$, and $y_{0}$, according to Eqs. (2.53)-(2.55),respectively. One usually sets the value of $y$ equal to zero by assuming that the laser frequency is close in value to that of the atomic transition frequency. The detuning effect can be taken into consideration by changing the value of the parameter $y_{0}$. This corresponds to changing the nondispersed cavity frequency. In practice, this can be done by modifying the distance between the two mirrors of a standing-wave laser resonator. Because the parameter $y_{0}$ is normalized to the atomic center transition frequency, $\omega_{0}$, see Eq. (2.55), the change in the value of $\Omega$ will cause the mode frequency that the laser cavity supports to be shifted away from the atomic center transition frequency, $\omega_{0}$ which is the meaning of detuning.

Let the detuning be represented by $\Delta v=x \Delta v_{h}$, where $\Delta v$ is frequency difference, $x$ is the detuning factor, $\Delta v_{h}=\frac{1}{\pi T_{2}}$ is the homogeneous linewidth, and $T_{2}$ is the coherence time. Therefore,

$$
\begin{gathered}
\Delta v=\frac{\Omega-\omega_{0}}{2 \pi}=x \Delta v_{h}, \\
\frac{2 \pi\left(\frac{\Omega-\omega_{0}}{2 \pi}\right)}{\gamma}=\frac{2 \pi x \Delta v_{h}}{\gamma}=\frac{2 \pi x \frac{1}{\pi T_{2}}}{\frac{1}{T_{2}}}, \\
y_{0}=2 x,
\end{gathered}
$$

where Eq. (2.55), $y_{0}=\frac{\left(\Omega-\omega_{0}\right)}{\gamma}$, is used. From Eq. (4.124) one sees that the amount of detuning can be changed by changing the value of detuning factor, $x$. The stability criteria for detuned lasers will be shown in the next chapter. 


\section{CHAPTER V}

\section{RESULTS}

\section{TYPE 1 (PERTURBATION) STABILITY CRITERIA}

As stated earlier, in order to determine the perturbation stability of the system under consideration, the real part of the normalized complex rate constant $s^{\prime}$ plays the important role. If the real part is negative, the steady state solution of the system is locally stable when subjected to an infinitesimal perturbation. The small perturbation, if introduced into the system will decay with time. If the real part of the complex rate constant is positive, on the other hand, the small perturbation that was introduced into the system will grow, and the steady state solution is locally unstable. By observing Eq. (4.120), one can see that the solutions of the equation are normalized complex rate constants as functions of the operating parameters. In our study we develop graphical representations that summarize the dynamic behaviors of laser systems based on the above perturbation analysis. The result for type 1 stability (perturbation stability) is shown in Figure 2.

From the figure, each curve is a boundary that separates the stable and unstable regions of operation in parameter space. The parameters $\delta, \rho$, and $r$ represent the ratio of polarization decay rate to cavity field decay rate, the ratio of population difference decay rate to polarization decay rate, and threshold parameter, respectively. The threshold at which a laser starts lasing corresponds to the threshold parameter, $r$ equal to one. Above each curve is where the real parts of the normalized complex rate constants $s^{\prime}$ are positive, so operation in that region is unstable. The small perturbation will grow with time if one 


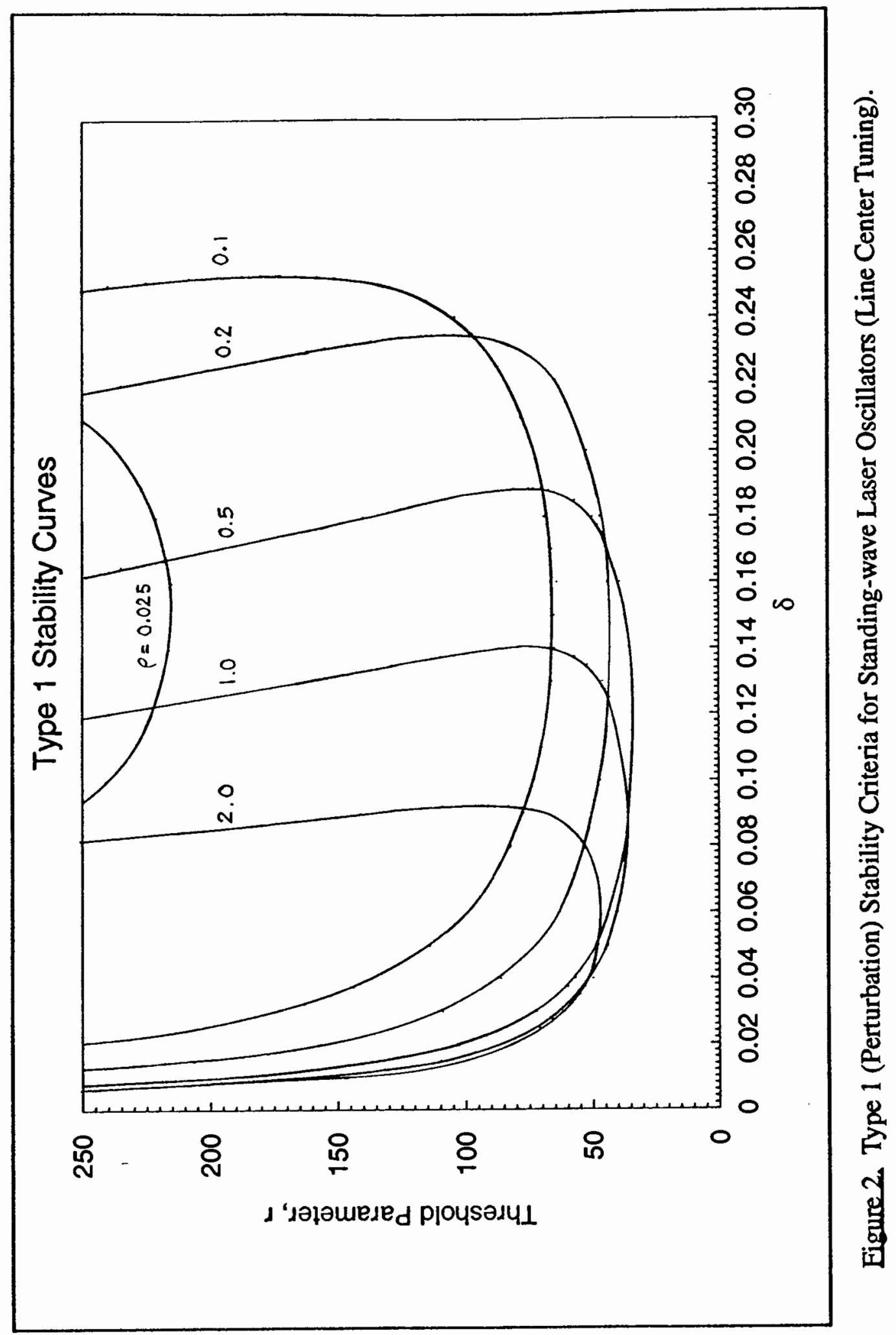


operates a laser in this region. Below the same curve, on the contrary, is where the real parts of the normalized rate constants are negative or the stable area. Linear stability analysis says that the small perturbation will die out with time for lasers operating in this region. One of the most interesting results regarding this figure is that the value of threshold parameter required to reach the unstable region is very high. The minimum value of the threshold parameter to reach an unstable boundary is about 34 times above the lasing threshold. Comparing this result with the result for the ring laser model ${ }^{(4)}$, also see Figure 3, which stated that the minimum value of threshold parameter to reach the perturbation boundary is 9 times above lasing threshold yields the conclusion that there is a greater possibility that one can observe pulsation behavior in the ring laser than in the standingwave laser. This assumption is based only on the interpretation of the type 1 stability curves which does not mean that one will not be able to observe pulsation phenomena in standingwave lasers at all. This conclusion has to be delayed until one gets the results of the type 2 stability curves, which are curves showing minimum values of the threshold parameter where pulsation phenomena are possible to be observed. The type 2 results will be presented in the next section.

\section{TYPE 2 STABILITY CRITERIA}

For this type of stability criteria, the results show curves in laser parameter space that correspond to the minimum values of the threshold parameter for which large amplitude oscillations will not decay with time. This threshold condition corresponds to a laser that initially is pulsing. The threshold parameter is then gradually reduced until the pulsations stop. The new criteria corresponding to the parameters used in Figure 2 are shown in Figure 4. The parameters $\delta, \rho$, and $r$ are as defined for the type 1 stability criteria. One can see that there is not much difference between the two types of stability criteria. The 


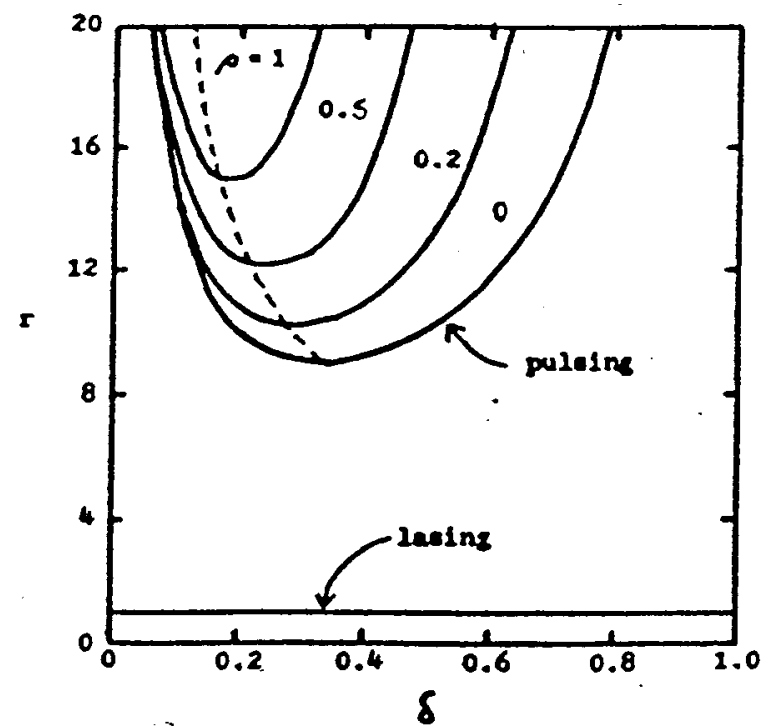

Eigure 3. Type 1 (Perturbation) Stability Criteria for Ring-laser Oscillators (Line Center Tuning). Source: [4].

minimum value of threshold parameter corresponds to this type 2 criteria is about 33 times above the lasing threshold. Now it is clear that the pulsation behavior in single-mode homogeneously broadened standing-wave lasers is difficult to observe. It requires a very high threshold value in addition to the "bad-cavity" condition. By comparing the result in Figure 4 with the ring laser result, Figure 5, the pulsation behavior in a ring laser can be observed outside the so-called "bad cavity" region, but in a standing-wave laser operating at line center this situation can not happen.

\section{DETUNING EFFECT}

The stability criteria of both type 1 and type 2 for detuned lasers are shown in Figure 6 and Figure 7. We choose the detuning value of a half of the homogeneous linewidth (detuning $=\Delta v_{h}$, where $\Delta v_{h}$ is the homogeneous linewidth), see Figure 8 , to 


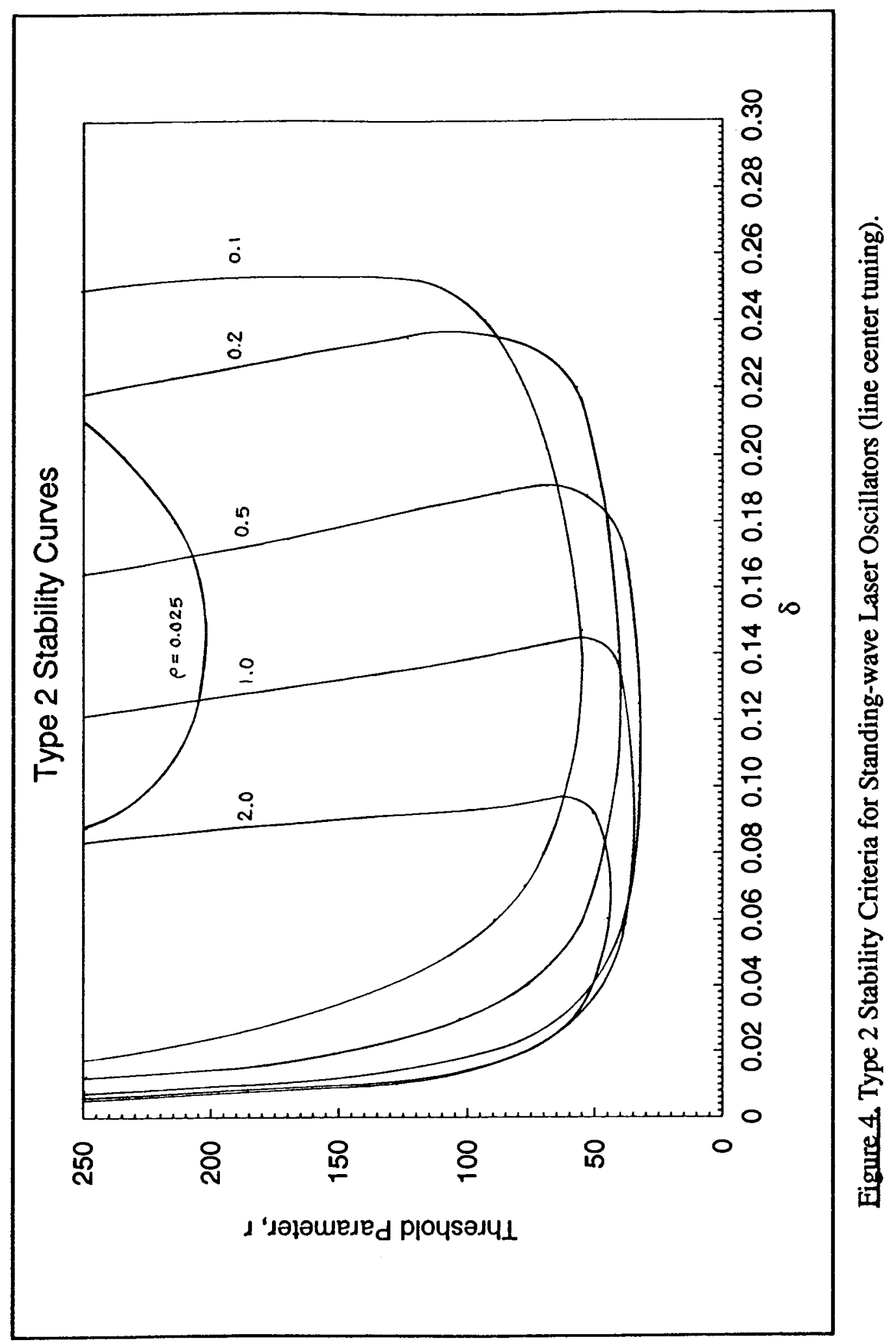




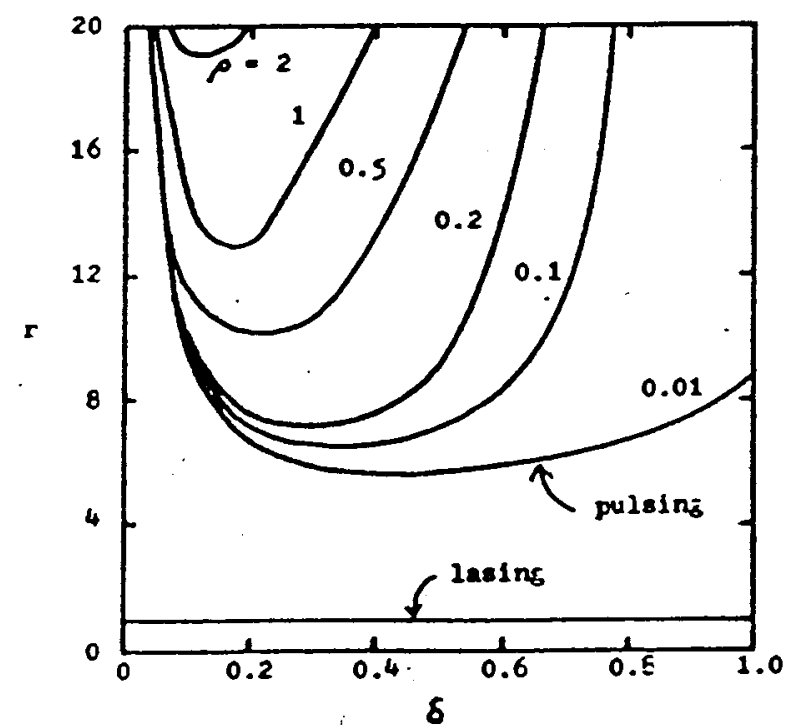

Eigure 5. Type 2 Stability Criteria for Ring-laser Oscillators (line center tuning). Source: [4].

coincide to the edge of the Lamb dip in inhomogeneously broadened standing-wave laser oscillators. At this particular position in the atomic gain profile, the gain of the laser drops to a half of the maximum value at the line center. This causes a change in the value of the threshold parameter that the laser needs to start lasing. For a detuning of half of the homogeneous linewidth, the value of the threshold parameter to start lasing is equal to two, which means that a laser needs to be pumped twice as hard as for line center operation because only half of the maximum gain value at line center is available.

From Figure 6 and Figure 7, one can observe that the effect of detuning is to raise the type 1 stability boundary and decrease the type 2 stability boundary. This similar effect was also reported for the ring laser model ${ }^{(33)}$. Because of the lower value of the type 2 stability boundary compared to that for line center tuning, a large amplitude induced 


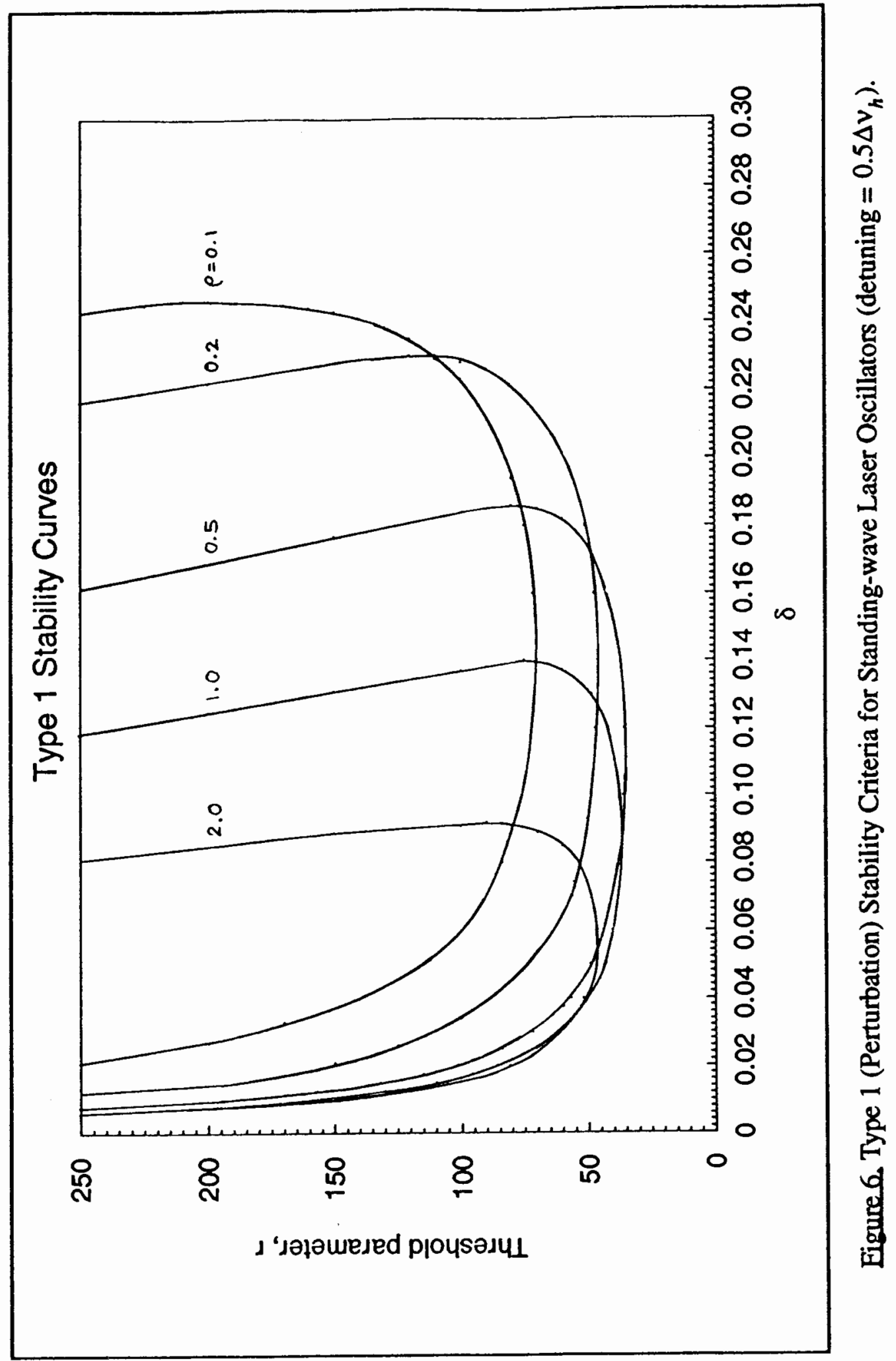




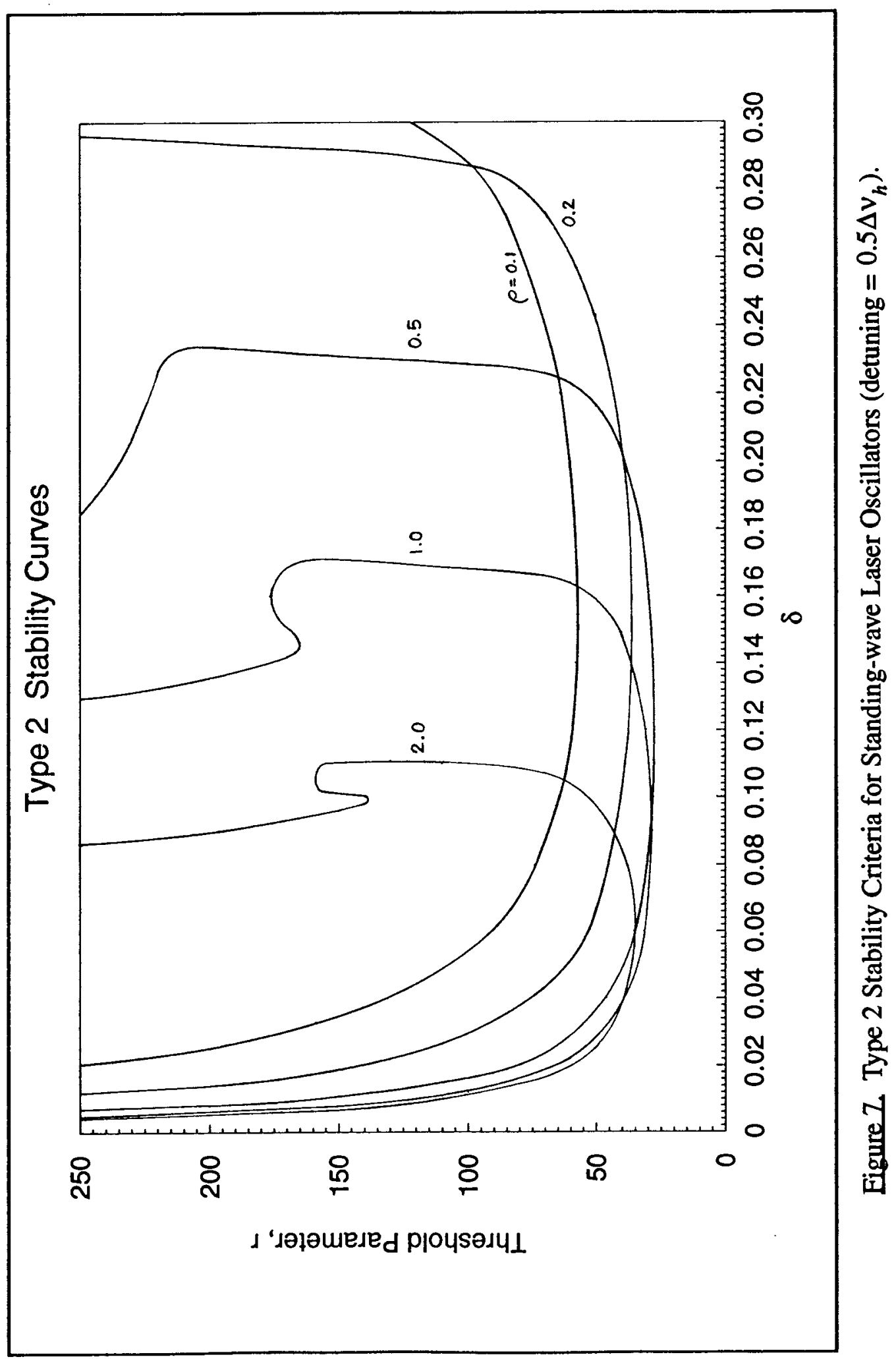


instability is expected to be observed experimentally easier in the detuned case. On the other hand, the increasing in the type 1 boundary is expected because increased pumping is also required to reach the lasing threshold.

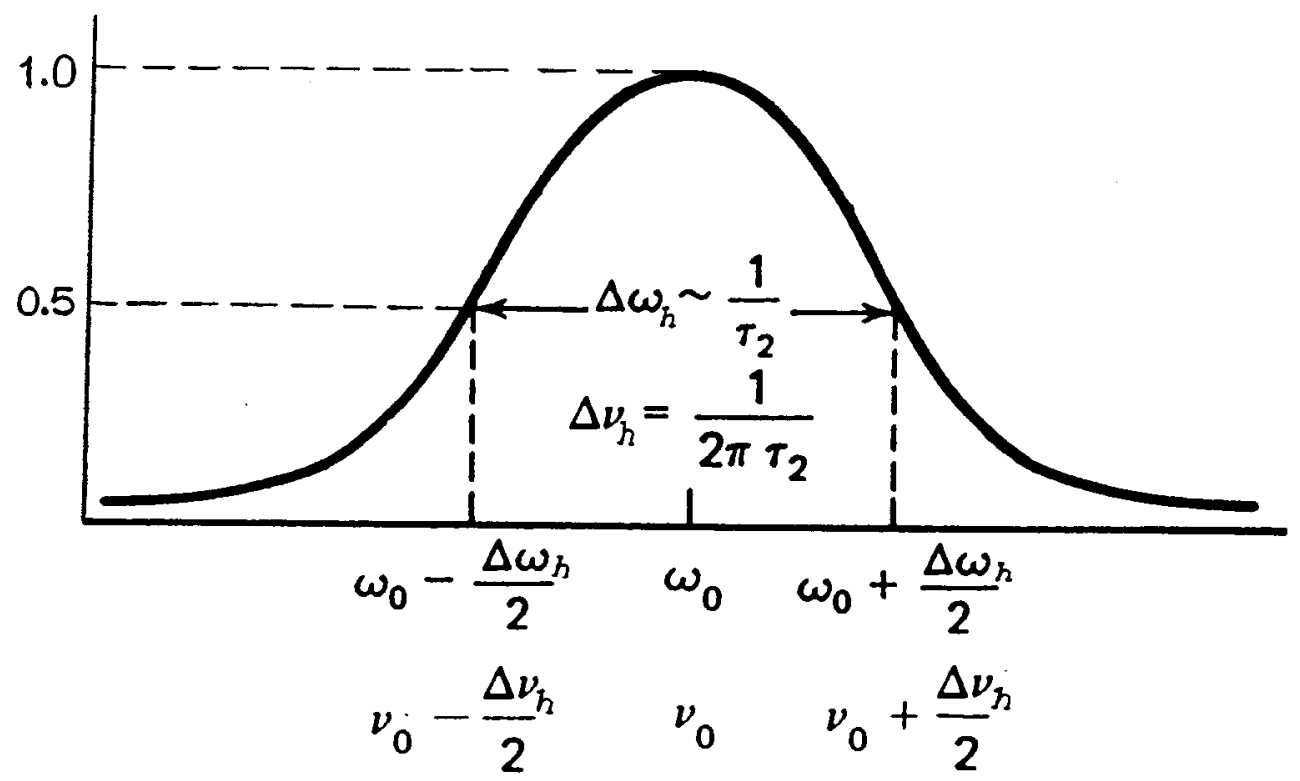

Eigure 8. Normalized Homogeneously Broadened Gain Profile $\left(\tau_{2}=\frac{T_{2}}{2}\right.$, $T_{2}=$ Coherence Time). Source: [34]. 


\section{CHAPTER VI}

\section{CONCLUSION}

In this study we have developed, for the first time, stability criteria for single mode strongly homogeneously broadened standing-wave laser oscillators. The results show a significant difference between the stability criteria of standing-wave laser oscillators and those obtained for ring-laser oscillators. In the case of line center tuning, we discover from type 1 stability criteria that undamped pulsations in single mode strongly homogeneously broadened standing-wave laser oscillators are found to occur at a much higher excitation level than in ring-laser oscillators with the same type of line broadening (at about thirty-four times above laser threshold as opposed to nine). The type 1 stability criteria also shows that in order to be able to observe the undamped pulsations, the "bad cavity" condition must be satisfied, especially for lasers that are operating at line center tuning. Another interesting feature that was observed is the effect of detuning on the stability criteria. It was found that detuning tends to raise the type 1 instability threshold and decrease the type 2 instability threshold as observed before in ring laser model ${ }^{(33)}$. This also leads to a wider "marginal" area. One might expect that at larger detuning, large amplitude induced undamped pulsations could be observed at a lower threshold value.

Future study about these topics can be pursued in several ways. Because the interesting effect of detuning on stability boundaries, the lower limit of the type 2 stability boundary as a function of detuning can be determined. By taking into account the effects of both homogeneous and inhomogeneous line broadening, one can obtain stability criteria for a large class of lasers, because all lasers, in practice, posses a combination of 
homogeneous and inhomogeneous line broadening. One can also investigate further the behavior of a laser as it makes the transition across the boundary from a stable region into an unstable region for different value of operating parameters. If one relaxes the plane wave approximation in a cavity field by considering the field with transverse variation, e.g. Gaussian profile, new stability criteria for the laser can also be obtained. 


\section{REFERENCES}

[1] R. G. Harrison and D. J. Biswas, "Pulsating Instabilities and Chaos in Lasers," Prog. Quant. Electr., Vol. 10, No. 3, pp. 147-228 (1985).

[2] N. B. Abraham, P. Mandel and L. M. Narducci, "Dynamical Instabilities and Pulsations in Lasers," in Progress in Optics, Vol. XXV, pp. 1-187 (1988).

[3] L. W. Casperson, in Third New Zealand Symposium on Laser Physics, J. D. Harvey and D. F. Walls, eds., Vol. 182 of Springer Lecture Notes in Physics (Springer-Verlag, Berlin, 1983), pp. 88-106.

[4] L. W. Casperson, "Spontaneous Coherent Pulsations in Ring-laser Oscillators: Stability Criteria," J. Opt. Soc. Am. B, Vol. 2, No. 6, pp. 993-997 (1985).

[5] A. Z. Grasiuk and A. N. Oraevskij, "The Dynamics of Quantum Oscillators," in Quantum Electronics and Coherent Light, edited by P. A. Miles, pp. 192-197, (Academic, New York, 1964).

[6] H. Risken and K. Nummedal, "Self-Pulsing in Lasers," J. Appl. Phys., Vol. 39, No. 10, pp. 4662-4672 (1968).

[7] S. T. Hendow and M. Sargent III, "Theory of Single Mode Laser Instabilities," J. Opt. Am. B, Vol. 2, No. 1, pp. 84-101 (1985).

[8] H. Haken, "Analogy Between Higher Instabilities in Fluids and Lasers," Phys. Letters, Vol. 53A, No. 1, pp. 77-78, (1975).

[9] E. N. Lorenz, "Deterministic Non-Periodic Flows," J. Atmos. Sci., Vol. 20, pp. 130-141, (1963). 
[10] C. Sparrow, The Lorenz Equations: Bifurcations, Chaos, and Strange Attractors, Vol. 41 of Springer Applied Mathematical Sciences Series,(Springer-Verlag, New York Inc., 1982).

[11] L. A. Lugiato and L. M. Narducci, "Single-mode and Multimode Instabilities in Lasers and Related Optical Systems," Phys. Rev. A, Vol. 32, No. 3, pp. 1576-1587 (1985).

[12] C. O. Weiss and W. Klische, "On Observability of Lorenz Instabilities in Lasers," Opt. Comm., Vol. 51, No. 1, pp. 47-48 (1984).

[13] C. O. Weiss, "Observation of Instabilities and Chaos in Optically Pumped Far-infrared Lasers," J. Opt. Am. B., Vol. 2, No. 1, pp. 137-140 (1985).

C. O. Weiss, W. Klische, P. S. Ering and M. Cooper, "Instabilities and Chaos of a Single-Mode $\mathrm{NH}_{3}$ Ring Laser," Opt. Comm., Vol. 52, No. 6, pp. 405-408 (1985).

[15] W. Klische and C. O. Weiss, "Instabilities and Routes to Chaos in a Homogeneously Broadened One- and Two-Mode Ring Laser," Phys. Rev. A., Vol. 31, No. 6, pp. 4049-4051 (1985).

[16] M. A. Dupertuis, R. R. E. Salomaa and M. R. Siegrist, "The Conditions for Lorenz Chaos in an Optically-Pumped Far-Infrared Laser," Opt. Comm., Vol. 57, No. 6, pp. 410-414 (1986).

[17] C. O. Weiss and J. Brock, "Evidence for Lorenz-Type Chaos in a Laser," Phys. Rev. Lett., Vol. 57, No. 22, pp. 2804-2806 (1986).

[18] P. Mandel and H. Zeghlache, "Stability of a Detuned Single Mode Homogeneously Broadened Ring Laser," Opt. Comm., Vol. 47, No. 2, pp. 146-150 (1983).

[19] H. Zeghlache and P. Mandel, "Influence of Detuning on the Properties of Laser Equations,” J. Opt. Am. B., Vol. 2, No. 1, pp. 18-22 (1985). 
[20] L. W. Casperson, "Spontaneous Coherent Pulsations in Ring-laser Oscillators," J. Opt. Am. B., Vol. 2, No.1, pp. 62-72 (1985).

[21] L. W. Casperson, "Spontaneous Coherent Pulsations in Standing-wave Laser Oscillators," J. Opt. Soc. Am. B, Vol. 2, No. 6, pp. 958-969 (1988).

[22] L. W. Casperson and M. F. H. Tarroja, "Spontaneous Coherent Pulsations in Standing-wave Laser Oscillators: Simplified Models," J.Opt. Soc. Am. B, Vol. 8, No. 2, pp. 250-261 (1991).

[23] L. W. Casperson, "Stability Criteria for High Intensity Lasers," Phys. Rev., Vol. 21, No. 3, pp. 911-923, (1980).

L. W. Casperson, "Stability Criteria for Non-Doppler Lasers," Phys. Rev., Vol. 23, No. 1, pp. 248-260, (1981).

[25] M. L. Minden and L. W. Casperson, "Dispersion-Induced Instability in CW lasers Oscillators," IEEE J. Quantum Electron., QE-18, pp. 1952-1957 (1982).

[26] M. L. Minden and L. W. Casperson, "Mode Splitting and the Coherent Instability in High-gain Lasers," J. Opt. Soc. Am. B, Vol. 2, No. 1, pp. 120-129 (1985).

[27] W. E. Lamb, Jr., "Theory of Optical Maser," Phys. Rev., Vol. 134, No. 6A, pp. A1429-A1450 (1964).

[28] M. Sargent III, M. O. Scully, and W. E. Lamb, Lr., Laser Physics, (AddisonWesley, 1974).

[29] L. W. Casperson, unpublished (1991).

[30] E. I. Gordon, A. D. White and J. D. Rigden, in Proceedings of the Symposium on Optical Masers, pp. 309-318 (Polytechnic Institute of Brooklyn, New York, 1963). 
[31] I. S. Gradshteyn and I. M. Ryzhik, Table of Integrals, series, and Products, Eq. 3.165.1, pp. 368, (Academic Press, 1979).

[32] M. Abramowitz and I. A. Stegun, Handbook of Mathematical Functions with Formulas, Graphs, and Mathematical Tables, Eq. 25.5.17, pp. 897, (Dover, 1970).

[33] L. W. Casperson, "Recent Progress in Modelling Single Mode Laser Instabilities," in Optical Instabilities edited by R. W. Boyd, M. G. Raymer and L. M. Narducci, Cambridge Studies in Modern Optics, Vol. 4, pp. 58-71, (Cambridge, 1986).

[34] J. T. Verdeyen, Laser Electronics, Second Edition, pp. 165, (Prentice-Hall, 1989).

[35] A. E. Siegman, Lasers, (University Science Books, Mill Valley, California, 1986). 